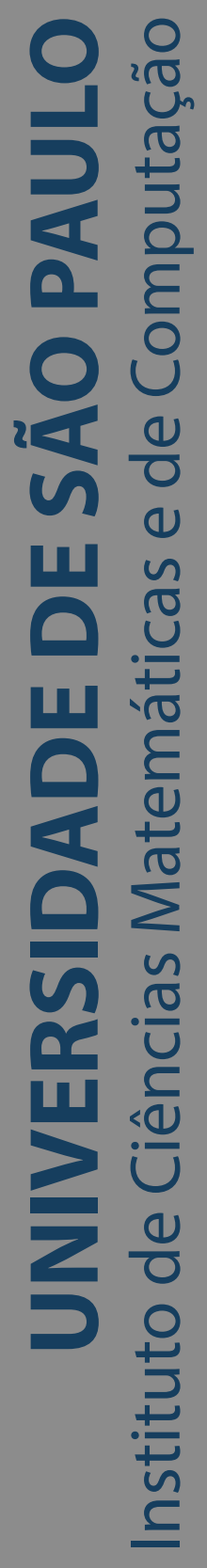

\title{
Design de campos vetoriais em volumes usando RBF
}

\section{Luiz Otávio Toratti}

Dissertação de Mestrado do Programa de Pós-Graduação em Ciências de Computação e Matemática Computacional (PPG-CCMC) 

Data de Depósito:

Assinatura:

\title{
Luiz Otávio Toratti
}

\section{Design de campos vetoriais em volumes usando RBF}

\author{
Dissertação apresentada ao Instituto de Ciências \\ Matemáticas e de Computação - ICMC-USP, \\ como parte dos requisitos para obtenção do título \\ de Mestre em Ciências - Ciências de Computação e \\ Matemática Computacional. VERSÃO REVISADA \\ Área de Concentração: Ciências de Computação e \\ Matemática Computacional \\ Orientadora: Prof. Dr. Afonso Paiva Neto
}


Ficha catalográfica elaborada pela Biblioteca Prof. Achille Bassi e Seção Técnica de Informática, ICMC/USP, com os dados inseridos pelo(a) autor(a)

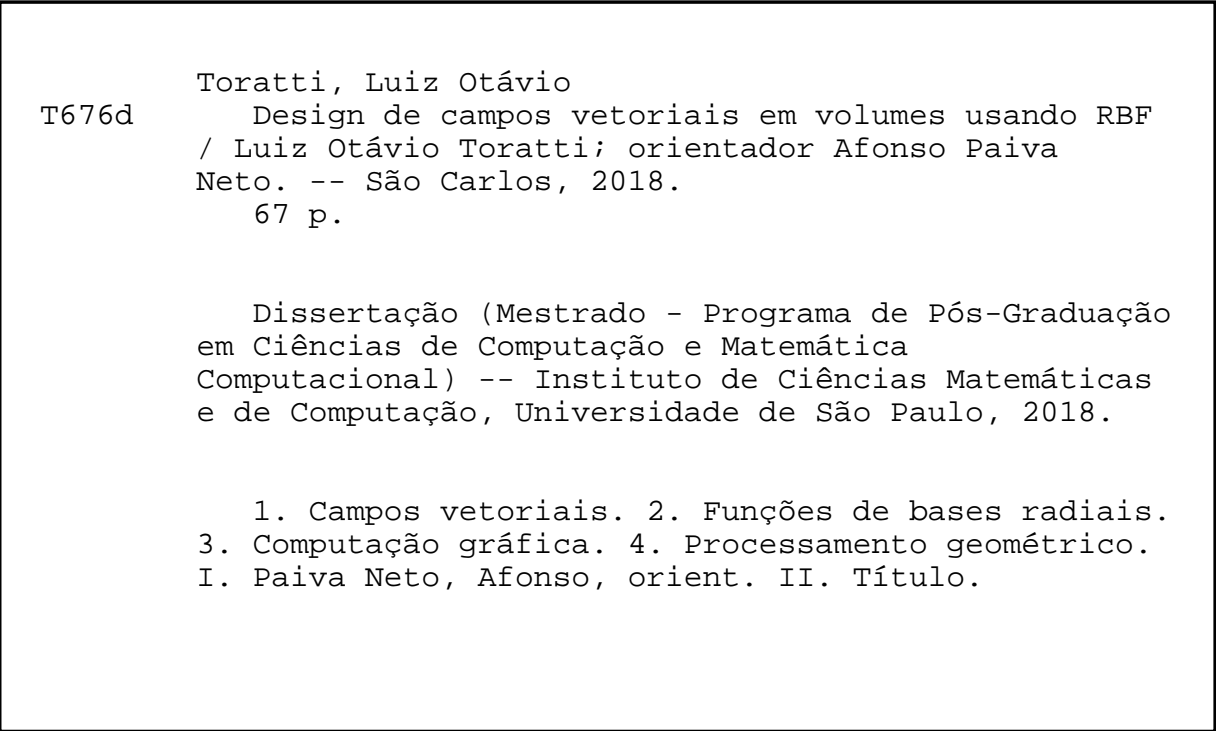

Bibliotecários responsáveis pela estrutura de catalogação da publicação de acordo com a AACR2: Gláucia Maria Saia Cristianini - CRB - 8/4938 Juliana de Souza Moraes - CRB - 8/6176 


\section{Luiz Otávio Toratti}

\section{Vector field design in volumes using RBF}

Master dissertation submitted to the Institute of Mathematics and Computer Sciences - ICMC-USP, in partial fulfillment of the requirements for the degree of the Master Program in Computer Science and Computational Mathematics. FINAL VERSION

Concentration Area: Computer Science and Computational Mathematics

Advisor: Prof. Dr. Afonso Paiva Neto 

Aos meus pais Osvaldo e Lúcia, pelo amor, apoio e dedicação que sempre tiveram por $\operatorname{mim}$.

À minha namorada e companheira de jornada que sempre me deu forças em todos os momentos.

Ao professor Afonso Paiva Neto pela orientação, amizade e paciência.

À todos os funcionários e professores do ICMC-USP por tornarem o instituto um lugar incrível.

À CAPES pelo apoio financeiro. 



\section{RESUMO}

TORATTI, L. O. Design de campos vetoriais em volumes usando RBF. 2018. 67 p. Dissertação (Mestrado em Ciências - Ciências de Computação e Matemática Computacional) - Instituto de Ciências Matemáticas e de Computação, Universidade de São Paulo, São Carlos - SP, 2018.

Em Computação Gráfica, campos vetoriais possuem diversas aplicações desde a síntese e mapeamento de texturas à animações de fluidos, produzindo efeitos amplamente utilizados na indústria do entretenimento. Para produzir tais campos, é preferível o uso de ferramentas de design em vez de simulações numéricas não só devido ao menor custo computacional mas, principalmente, por prover liberdade ao artista ao sintetizar o campo de acordo com a sua necessidade. Atualmente, na literatura, existem bons métodos de design de campos vetoriais em superfícies de objetos tridimensionais porém, o design no interior desses objetos ainda é pouco estudado, principalmente quando o campo de interesse possui propriedades específicas. O objetivo deste trabalho é desenvolver uma técnica para sintetizar campos vetoriais, com características do movimento de fluidos incompressíveis, no interior de domínios. Em uma primeira etapa, o método consiste na interpolação dos vetores de controle, com uma certa propriedade desejada, em todo o domínio. Posteriormente, o campo obtido é modificado para respeitar a geometria do contorno.

Palavras-chave: Campos vetoriais, Funções de base radial, Fast Marching, Computação gráfica, Processamento geométrico. 



\section{ABSTRACT}

TORATTI, L. O. Vector field design in volumes using RBF. 2018. 67 p. Dissertação (Mestrado em Ciências - Ciências de Computação e Matemática Computacional) - Instituto de Ciências Matemáticas e de Computação, Universidade de São Paulo, São Carlos - SP, 2018.

Vector fields are important to an wide range of applications on the field of Computer Graphics, from the synthesis and mapping of textures to fluid animation, producing effects widely used on the entertainment industry. To produce such fields, design tools are prefered over numerical simulations not only for its lower computational cost, but mainly by providing freedom to the artist in the creation process. Nowadays, good methods of vector field design over surfaces exist in literature, however there is only a few studies on the synthesis of vector fields of the interior of objects and even fewer when specific properties of the field are required. This work presents a technique to synthesize vector fields with properties of imcompressible fluid's motion in the interior of objects. On a first step, the method consists in interpolating control vectors with a certain desired property throughout the whole domain and later the resulting field is modified to properly fit the boundary geometry of the object.

Keywords: Vector fields, Radial base functions, Fast Marching, Computer graphics, Geometric processing. 



\section{LISTA DE ILUSTRAÇÕES}

Figura 1 - Mapeamento de textura na superfície utilizando o campo vetorial obtido por restrições dadas por curvas direcionais (esquerda) e pontos de singularidade (direita) . . . . . . . . . . . . . . . . . .

Figura 2 - Design de campo vetorial no volume de um bitorus. A esquerda, o campo é controlado pelas curvas amarela e azul. A direita, o campo obtido é utilizado para sintetizar elementos no objeto.

Figura 3 - A esquerda o campo vetorial é produzido com um par de fontes e a direita o campo é remodelado pela restrição da curva. . . . . . . . . . . . . . . . 20

Figura 4 - Campo vetorial produzido especificando-se os pontos de singularidade. . . . 20

Figura 5 - Malha hexaedral gerada com o auxilío de um campo octaedral. . . . . . . . 21

Figura 6 - Campo tensorial aplicado em síntase de elementos. O campo é modelado através da edição das curvas coloridas (figura esquerda). Síntese de elementos com o campo obtido (figura direita). . . . . . . . . . . . . . . . . 21

Figura 7 - Exemplos de funções de base radial . . . . . . . . . . . . . 25

Figura 8 - Variação do parâmetro de forma $\varepsilon$ na interpolação dos dados quando utilizada a função $\phi(r)=e^{-\varepsilon r^{2}}$. Nesse caso, quando aumentamos o valor de $\varepsilon$ estamos diminuindo a região de influência . . . . . . . . . . . . . . 25

Figura 9 - Exempolo bidimensional de $\Phi_{\text {div }} \operatorname{com} \phi_{3,3} \ldots \ldots$. . . . . . . . . . . 30

Figura 10 - Interpolações utilizando os núcleos $\Phi_{\text {div }}$ e $\Phi_{\text {curl }}$. . . . . . . . . . . . . . 31

Figura 11 - Mapa de distâncias, a partir de um ponto, representado pela escala de cores. 32

Figura 12 - Vizinhança em $\mathbb{R}^{2} \ldots \ldots \ldots \ldots$. . . . . . . . . . . 32

Figura 13 - Soluções da equação Eikonal através do método FMM. A escala de cores corresponde as distâncias. . . . . . . . . . . . . . . . . . 34

Figura 14 - Mapa da distância interior a partir de um ponto. . . . . . . . . . . . . . 35

Figura 15 - Interpolação vetorial, por componente, na esfera. . . . . . . . . . . . 38

Figura 16 - Interpolação vetorial, por componente, numa núvem de pontos do modelo bunny. . . . . . . . . . . . . . . . . . 39

Figura 17 - Interpolação dos vetores de controle na núvem de pontos, utilizando os núcleos $\Phi_{d i v}$ e $\Phi_{c u r l} \ldots \ldots \ldots$. . . . . . . . . . . . . . . . .

Figura 18 - Interpolação simultânea dos vetores de controle no interior e do campo tangente no contorno . . . . . . . . . . . . . . . 43

Figura 19 - Funções peso construídas a partir da distância da fronteira. . . . . . . . . . . 45

Figura 20 - Exemplo do método proposto . . . . . . . . . . . . . . . . 46 
Figura 21 - Influência da vizinhança no método de quasi-interpolação . . . . . . . . . . 47

Figura 22 - Restrição do suporte . . . . . . . . . . . . . . . . . 48

Figura 23 - Comportamento da distância ao aproximar regiões não convexas . . . . . . 50

Figura 24 - Influência da distância no núcleo . . . . . . . . . . . . . . . 51

Figura 25 - Tentativa de contruir o campo $F_{c t r}$ com a distância de Rustamov acoplada . . 51

Figura 26 - Média das magnitudes das derivadas . . . . . . . . . . . . . . 52

Figura 27 - Malha triangular do domínio . . . . . . . . . . . . . . 52

Figura 28 - Exemplo do método proposto com a distância do FMM acoplada. . . . . . . 53

Figura 29 - Exemplo do método proposto num domínio mais suave. . . . . . . . . . . . 54

Figura 30 - Influência no campo final quando alteramos a distância na construção de $F_{c t r}$. 56

Figura 31 - Exemplo de um domínio não convexo em que não há diferenças visuais significativas em utilizar a $d_{F M M} \ldots \ldots \ldots \ldots$

Figura 32 - Domínio com algumas melhorias visuais, porém com maior custo computaci-

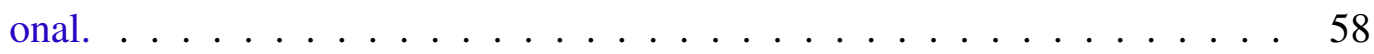

Figura 33 - Testes com as duas abordagens propostas. As figuras da esquerda, utilizam a distância euclidiana $\|\cdot\|_{2}$ na construção do campo $F_{c t r}$. Já as da direita utilizam a distância $d_{F M M} \ldots \ldots \ldots$. . . . . . . . . . . . 59

Figura 34 - Primeiro resultado tridimensional utilizando uma esfera como domínio . . . 63 


\section{LISTA DE TABELAS}

Tabela 1 - Exemplos de algumas RBFs tradicionais . . . . . . . . . . . . . . 24

Tabela 2 - CSRBFs de Wendland . . . . . . . . . . . . . . 26

Tabela 3 - Resultados obtidos com as métricas: $\|\cdot\|_{2}$ e $d_{F M M}$ no cálculo do campo $F_{c t r}$. 57 

INTRODUÇÃO . . . . . . . . . . . . . . . . 17

TRABALHOS RELACIONADOS . . . . . . . . . . . . . . 19

CONCEITOS BÁSICOS . . . . . . . . . . . . . . . . 23

$3.1 \quad$ Funções de Bases Radiais (RBF) . . . . . . . . . . . . . . 23

3.1.1 Interpolação via RBF . . . . . . . . . . . . . . . . 23

3.1.2 RBFs com Suporte Compacto . . . . . . . . . . . . . . 26

3.2 Interpolação via Núcleos Matriciais . . . . . . . . . . . . 26

3.2.1 Interpolação com Núcleos Matriciais de Funções de Base Radial . . 27

3.2.2 Núcleos MVRBF com Divergente Nulo e Rotacional Nulo . . . . . . 28

$3.3 \quad$ Métrica Restrita $\times$ Métrica Intrinseca . . . . . . . . . . . 31

3.3.1 Métricas Intrínsecas e Funções de Bases Radiais . . . . . . . . . . . 32

3.3.2 Distância Geodésica Discreta via FMM . . . . . . . . . . . . . . . . 33

3.3.3 Distância Interior usando Coordenadas Baricêntricas . . . . . . . . . 34

4 INTERPOLANDO CAMPOS VETORIAIS EM SUPERFÍCIE . . . . 37

4.1 Interpolação com RBF por Componente . . . . . . . . . . . 37

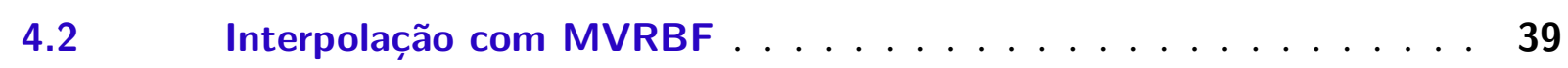

5 ABORDAGEM PROPOSTA ................... 41

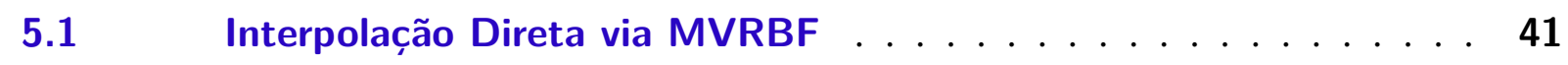

$5.2 \quad$ Método Proposto ................... 43

5.3 Restringindo o Domínio das RBFs . . . . . . . . . . . . . 45

$5.4 \quad$ Acoplando Distância Interior em Núcleos MVRBF . . . . . . . . 48

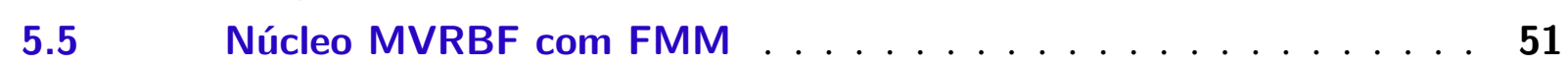

$5.6 \quad$ Considerações $\ldots \ldots \ldots \ldots \ldots \ldots$

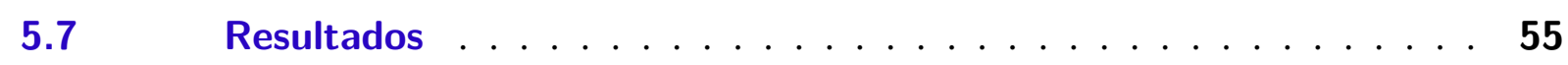

6 CONCLUSÕES E PERSPECTIVAS . . . . . . . . . . 61

REFERÊNCIAS . . . . . . . . . . . . . . . . . . 65 



\section{1}

\section{INTRODUÇÃO}

Campos vetoriais possuem um papel fundamental na formulação de diversos conceitos físicos e matemáticos, tais como campos magnéticos, campos gravitacionais e campos de velocidades de fluídos. $\mathrm{Na}$ indústria do entretenimento, como filmes e jogos eletronicos, os campos vetoriais são utilizados para produzir diversos efeitos visuais e, em particular, muitas animações são baseadas em movimentos de fluídos. O fluxo de fluídos é bem descrito por equações matemáticas e as simulações numéricas dessas equações são amplamente utilizadas em diversas áreas da engenheria. Em Computação Gráfica, o uso dessas simulações geram ótimos resultados em animações porém, o elevado custo computacional decorrente da discretização das equações torna várias aplicações inviáveis.

A indústria do entretenimento têm um grande interesse em ferramentas de boa produtividade, de fácil utilização e que produzam bons resultados visuais, tornando a alta precisão obtida em simulações menos relevante. Além do custo computacional, outro fator importante a ser considerado, é a possibilidade de modelagem e edição dos campos, ou seja, o design do artista. Ajustar parâmetros em equações diferenciais não é um processo de controle intuitivo para o animador. Com isso, o mercado profissional busca por métodos alternativos para construir campos vetoriais sintéticos, intuitivo para usuários sem os conhecimentos físicos e matemáticos envolvidos. Atualmente, o estudo de ferramentas para design de campos vetoriais vem ganhando destaque devido ao grande crescimento deste mercado. Duas ótimas referências são: o trabalho de Fisher et al. (2007), apresentando um eficiente método de design de campos vetoriais em superfícies de malhas triangulares com aplicação em mapeamento de texturas, ilustrado na Figura 1, e o recente trabalho de Palacios et al. (2016), para design em volumes, Figura 2.

O objetivo desse trabalho é desenvolver um método de design de campos vetoriais em volumes que respeite a fronteira do objeto (que tangencie o domínio dado) e possuam divergência nula, para aplicações especialmente em Computação Gráfica. Fisicamente, a propriedade de divergência nula em campos vetoriais modela matematicamente a preservação do volume de 
Figura 1 - Mapeamento de textura na superfície utilizando o campo vetorial obtido por restrições dadas por curvas direcionais (esquerda) e pontos de singularidade (direita).

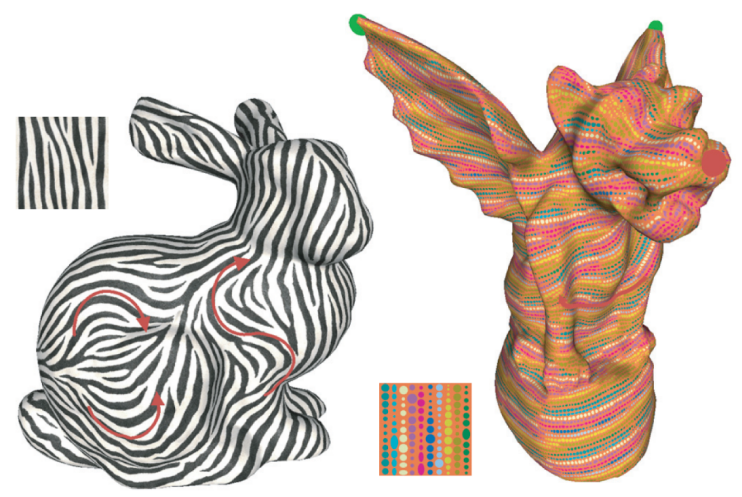

Fonte: Fisher et al. (2007).

Figura 2 - Design de campo vetorial no volume de um bitorus. A esquerda, o campo é controlado pelas curvas amarela e azul. A direita, o campo obtido é utilizado para sintetizar elementos no objeto.
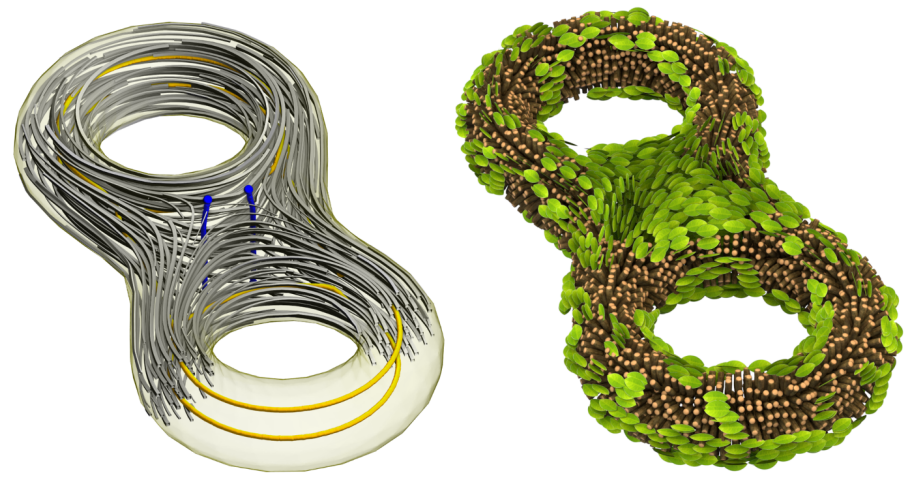

Fonte: Palacios et al. (2016).

fluidos em superfícies fechadas, definindo uma característica visual extremamente importante no movimento de fluídos comuns como ar, água e fumaça. Tal propriedade motivou a escolha da principal ferramenta matemática utilizada: as Funções de Base Radial, do inglês, Radial Basis Function (RBF). O uso de RBF's é muito popular em problemas de interpolação de dados multidimensionais por ser um ferramenta poderosa e de fácil implementação mas também, com elas podemos construir interpolantes para campos vetoriais, resultando em campos vetoriais com divergente nulo ou rotacional nulo.

No texto que segue, no Capítulo 2 é apresentada uma revisão bibliográfica de alguns trabalhos relacionados ao problema estudado. No Capítulo 3, são abordados os conceitos fundamentais e ferramentas matemáticas utilizadas no método proposto. O Capítulo 4 trás uma aplicação dessas ferramentas com o design de campos vetoriais em uma núvem de pontos tridimensional. No Capítulo 5, é apresentado o método proposto deste trabalho juntamente com os resultados obtidos e finalmente, no Capítulo 6, seguem as conclusões finais, bem como as perspectivas para trabalhos futuros. 


\section{TRABALHOS RELACIONADOS}

Neste capítulo, apresentamos alguns trabalhos relacionados ao problema abordado com exceção dos trabalhos referentes às ferramentas utilizadas pois, serão apresentadas com detalhes no próximo capítulo. Como dito anteriormente, o design de campos vetoriais possui inúmeras aplicações em computação gráfica e processamento geométrico tais como, síntese de texturas, renderização, deformações, animações e muitas outras. A síntese desses campos podem variar de acordo com a aplicação pois, possuem diferentes objetivos, representações e propriedades específicas.

Este trabalho toma como ponto de partida o problema de interpolação/reconstrução de campos vetoriais. Em termos mais gerais, através de medidas de um fenômeno real, experimentos ou simulações, os campos vetoriais podem ser amostrados em conjuntos finitos de dados e então, extrapolados em toda uma região desejada. Em Lage et al. (2006), é proposto um método de reconstrução de campos vetoriais $2 \mathrm{D}$ a partir de um conjunto esparso de dados não estruturados. Basicamente, o algoritmo proposto, faz aproximações polinomiais locais e então utiliza o método da partição da unidade para construir a aproximação global. Já o trabalho de Lage et al. (2009), também trata de um método para reconstrução de campos vetoriais de dados esparsos porém, no contexto de aprendizado de máquina. Em Macêdo e Castro (2008), os autores utilizam a teoria do aprendizado estatístico com certos núcleos matriciais, garantindo propriedades de divergente livre e rotacional livre no campo recontruido.

Em direção aos trabalhos de design de campos, Bridson, Houriham e Nordenstam (2007) apresentam uma forma simples e eficiente de gerar campos turbulentos de velocidade. A partir de uma função potencial, o campo é construido do rotacional dessa função e, portanto, terá divergente nulo. Porém, para o campo potencial de entrada, é utilizado uma função de ruído e, por mais que seja possível fazer modulações gerando novos potenciais a fim de modificar o campo, não há a perda da aleatoriedade do campo resultante. Já Fisher et al. (2007), apresentam um método de design de campos tangentes em malhas triangulares arbitrárias, muito eficiente e de fácil edição, onde o fluxo do campo pode ser controlado por curvas sobre a superfície e 
as fontes podem ser adicionadas apenas escolhendo sua localização (veja Figura 3). Utilizando conexões em superfícies discretas, Crane, Desbrun e Schröder (2010) apresentam seu método para design de campos em superfícies possibilitando ao usuário inserir exatamente os únicos pontos de singularidade e controlar a direção com curvas, conforme ilustrado na Figura 4.

Figura 3 - A esquerda o campo vetorial é produzido com um par de fontes e a direita o campo é remodelado pela restrição da curva.
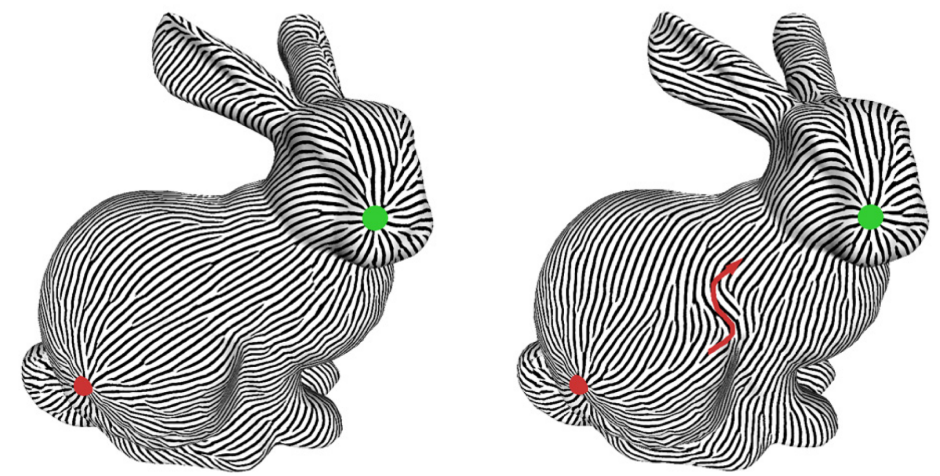

Fonte: Fisher et al. (2007).

Figura 4 - Campo vetorial produzido especificando-se os pontos de singularidade.

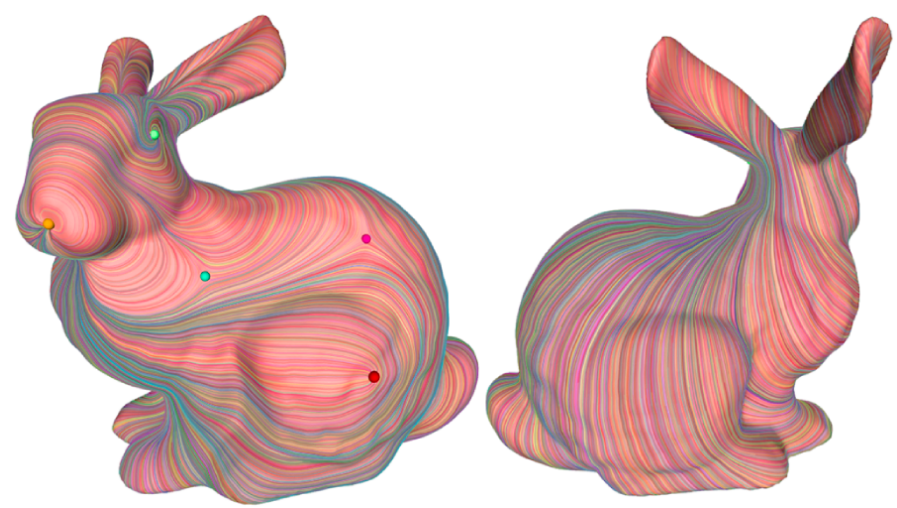

Fonte: Crane, Desbrun e Schröder (2010).

Outros métodos de design de campos vetoriais em superfícies podem ser encontrados em Vaxman et al. (2017). Porém, o design em volumes ainda foi pouco tratado. Recentemente, Solomon, Vaxman e Bommes (2017) calculam um campo vetorial octaedral, suave, no volume de acordo com restrições dadas apenas na fronteira de uma malha triangular com aplicações em geração de malhas hexaedrais (vide Figura 5). Já Palacios et al. (2016) apresentam um sistema interativo para design de campos tensoriais no interior de objetos tridimensionais. O usuário especifica valores desejados do campo e padrões locais dentro do volume e/ou na superfície de contorno e, a partir desses dados, pode-se modelar a topologia do campo tensorial através da edição de curvas de controle. A Figura 6 mostra um resultado obtido deste método, em que o campo resultante é aplicado em síntase de elementos. Porém, as edições topológicas podem falhar e o motivo, até o momento, não possui explicações conclusivas. 
Figura 5 - Malha hexaedral gerada com o auxilío de um campo octaedral.

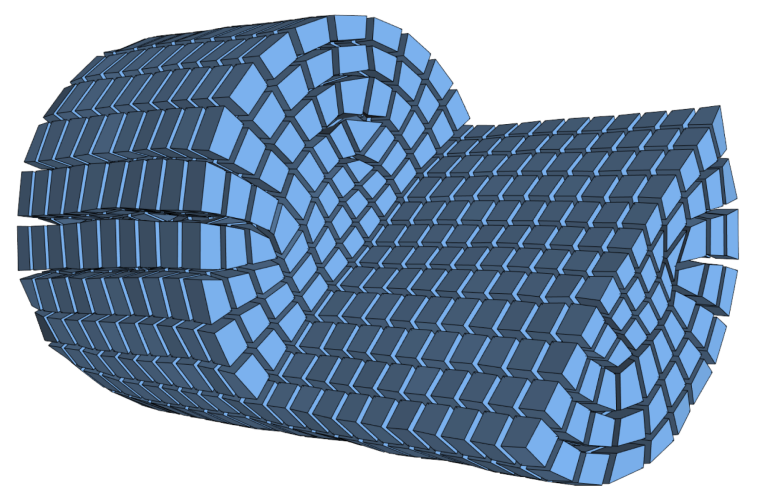

Fonte: Solomon, Vaxman e Bommes (2017).

Figura 6 - Campo tensorial aplicado em síntase de elementos. O campo é modelado através da edição das curvas coloridas (figura esquerda). Síntese de elementos com o campo obtido (figura direita).
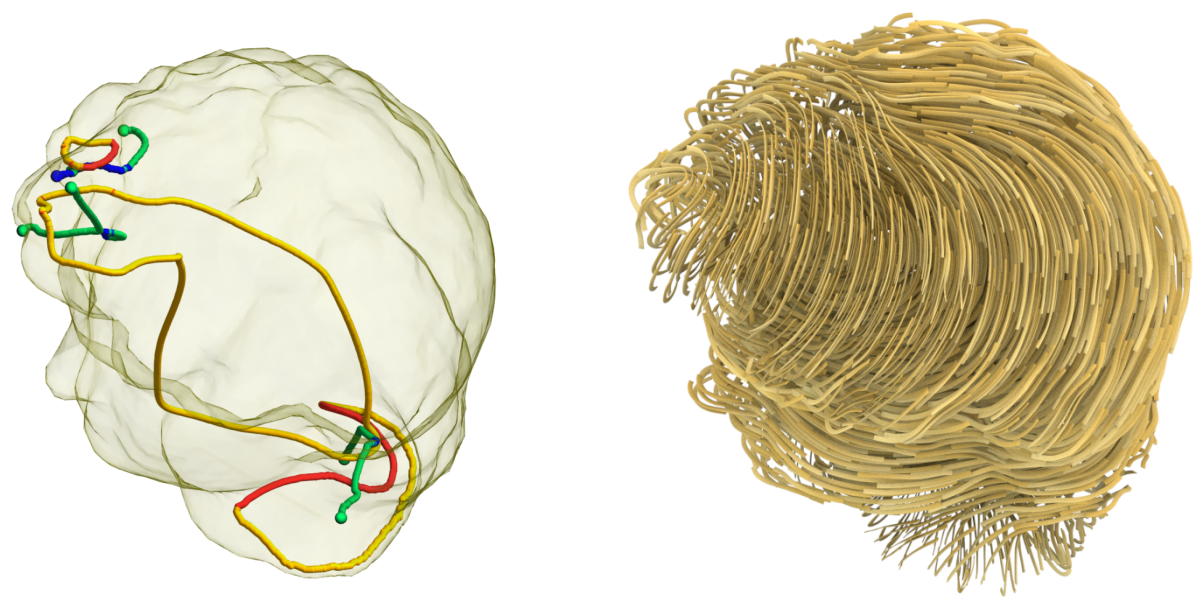

Fonte: Palacios et al. (2016). 



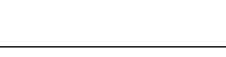

\section{CONCEITOS BÁSICOS}

Neste capítulo, são apresentados de forma sucinta, os fundamentos matemáticos e computacionais utilizados nesse trabalho tais como: o uso de funções escalares de bases radiais para construir interpolantes de campos vetoriais com divergente nulo e métodos numéricos para produzir campos de distâncias geodésicas em regiões poligonais do plano.

\subsection{Funções de Bases Radiais (RBF)}

As RBFs constituem uma família de funções amplamente utilizadas em problemas de interpolação de dados multidimensionais. Provavelmente, foram introduzidas, no contexto computacional, na década de 70 e desde então, sua utilização tem se tornado muito popular nas mais diversas áreas como Computação Gráfica, Aprendizado de Máquina e Mecânica de Fluidos Computacional. Nesta seção, são introduzidos os conceitos fundamentais dessa ferramenta através do problema tradicional de interpolação de dados escalares.

\subsubsection{Interpolação via RBF}

Considere o seguinte problema de interpolação: dado um conjunto de pontos distintos $X=\left\{x_{1}, \ldots, x_{N}\right\} \subset \mathbb{R}^{d}$ e um conjunto de valores reais $f_{1}, \ldots, f_{N}$ associdados respectivamente a esses pontos, encontrar uma função contínua $s_{f}: \mathbb{R}^{d} \rightarrow \mathbb{R}$ tal que $s_{f}\left(x_{i}\right)=f_{i}, 1 \leq i \leq N$. Uma abordagem comum para esse problema consiste em assumir que a função interpoladora seja dada por uma combinação linear de certas funções de base $\Phi_{j}: \mathbb{R}^{d} \rightarrow \mathbb{R}, j=1,2, \ldots, N$, ou seja, a função interpolante possui a seguinte forma

$$
s_{f}(x)=\sum_{j=1}^{N} \Phi_{j}(x) \alpha_{j}
$$


em que $\left\{\alpha_{i}\right\}_{i=1}^{N}$ são certos coeficientes reais. Aplicando as condições de interpolação, $s_{f}\left(x_{i}\right)=f_{i}$, $1 \leq i \leq N$, na Equação 3.1,

$$
f_{i}=f\left(x_{i}\right)=\sum_{j=1}^{N} \Phi_{j}\left(x_{i}\right) \alpha_{j}, \quad i=1,2, \ldots, N
$$

obtemos um sistema linear com $N$ equações e $N$ incógnitas

$$
\left[\begin{array}{cccc}
\Phi_{1,1} & \Phi_{1,2} & \cdots & \Phi_{1, N} \\
\Phi_{2,1} & \Phi_{2,2} & \cdots & \Phi_{2, N} \\
\vdots & \vdots & \ddots & \vdots \\
\Phi_{N, 1} & \Phi_{N, 2} & \cdots & \Phi_{N, N}
\end{array}\right]\left[\begin{array}{c}
\alpha_{1} \\
\alpha_{2} \\
\vdots \\
\alpha_{N}
\end{array}\right]=\left[\begin{array}{c}
f_{1} \\
f_{2} \\
\vdots \\
f_{N}
\end{array}\right]
$$

em que $\Phi_{i, j}=\Phi_{j}\left(x_{i}\right)$.

A existência e unicidade do interpolante dependem da escolha das funções de base $\Phi_{j}$, desta forma, são utilizadas funções definidas positivas pois, garantem a não singularidade da matriz de interpolação. Dizemos que uma função $\Phi: \mathbb{R}^{d} \rightarrow \mathbb{R}$ é definida positiva se para todo $N \in$ $\mathbb{N}$, todo par distinto $x_{1}, \ldots, x_{N} \in \mathbb{R}^{d}$, e todo $\alpha \in \mathbb{R}^{N} \backslash\{0\}$, a forma quadratica $\sum_{j, k=1}^{N} \alpha_{j} \alpha_{k} \Phi\left(x_{j}-\right.$ $x_{k}$ ) é positiva. As funções positivas definidas são bem caracterizadas por transformadas de Fourier e relacionadas com um tipo especial de funções, as funções radiais. Uma função $\Phi: \mathbb{R}^{d} \rightarrow \mathbb{R}$ é dita ser radial se existir uma função $\phi:[0, \infty) \rightarrow \mathbb{R}$ tal que $\Phi(x)=\phi\left(\|x\|_{2}\right)$ para todo $x \in \mathbb{R}^{d}$. Embora a definição mais comum venha a sugerir a utilização da norma euclidiana, funções do tipo $\ell_{p}$-radial da forma $\Phi(x)=\phi\left(\|x\|_{p}\right), x \in \mathbb{R}^{d}$ também poderiam ser consideradas no problema de interpolação mas, não receberam importância até o momento. Assim, Wendland (2005), motiva a construção e utilização das funções radiais definidas positivas, tornando-as uma ótima escolha como funções de base no problema de interpolação. Em termos matemáticos, tomamos como funções de base $\Phi_{j}(x)=\phi\left(\left\|x-x_{j}\right\|_{2}\right)$ para alguma $\phi:[0, \infty) \rightarrow \mathbb{R}$ definida definida. Na Tabela 1 são sumarizadas algumas das RBF's mais utilizadas, enquanto que a Figura 7 ilustra dois exemplos de funções de base $\phi: \mathbb{R}^{2} \rightarrow \mathbb{R}$.

Tabela 1 - Exemplos de algumas RBFs tradicionais

\begin{tabular}{c|c}
\hline RBF & $\phi(r), r=\|x\|_{2}$ \\
\hline Gaussiana & $e^{-\varepsilon r^{2}, \varepsilon}>0$ \\
Multiquádrica & $\sqrt{r^{2}+\varepsilon^{2}}, \varepsilon>0$ \\
Thin Plate Splines & $r^{2} \log (r)$ \\
Triharmônica & $r^{3}$ \\
Wendland & $\phi_{n, k}$, Tabela 2 \\
\hline
\end{tabular}

As RBF's dependem da distância do ponto de avaliação $x$ a um centro fixado $x_{j}$, criando uma região de influência ao redor deste ponto. Para controlar essa região de influência, a grande maioria das RBF's possuem um parâmetro de controle $\varepsilon$ conhecido como parâmetro de forma. A escolha correta deste parâmetro é parte fundamental do problema pois, afeta drasticamente 
Figura 7 - Exemplos de funções de base radial

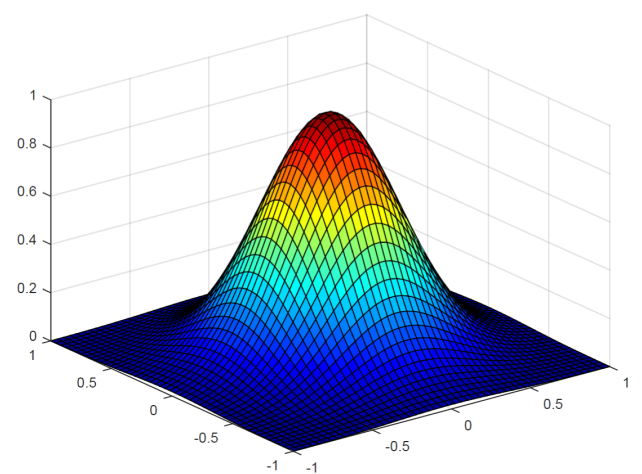

(a) Gaussiana $\phi(r)=e^{-\varepsilon r^{2}}, \varepsilon=4.0$

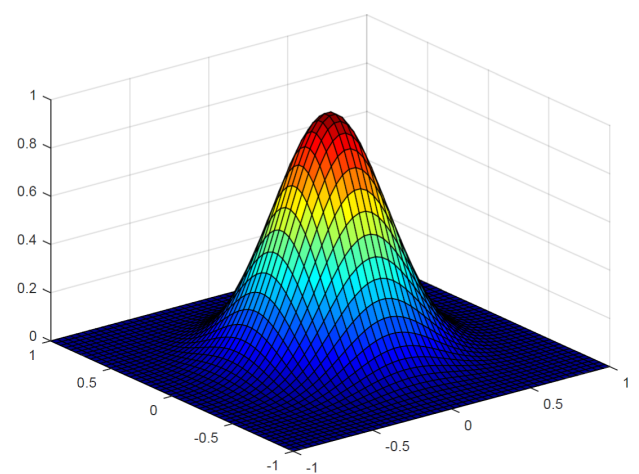

(b) Wendland $\phi_{3,3}(r), \quad \varepsilon=0.75$

Figura 8 - Variação do parâmetro de forma $\varepsilon$ na interpolação dos dados quando utilizada a função $\phi(r)=e^{-\varepsilon r^{2}}$. Nesse caso, quando aumentamos o valor de $\varepsilon$ estamos diminuindo a região de influência

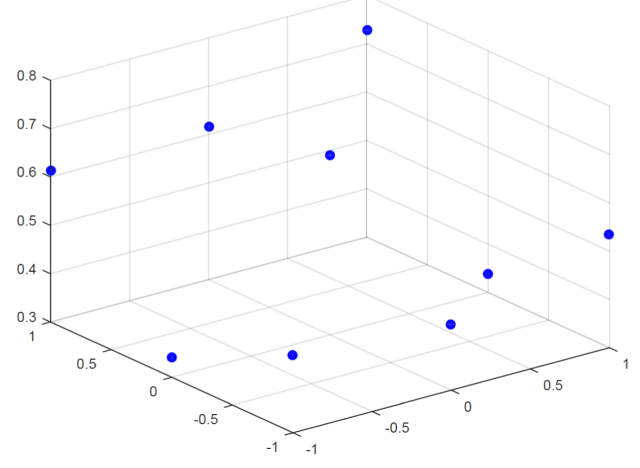

(a) Conjunto de dados

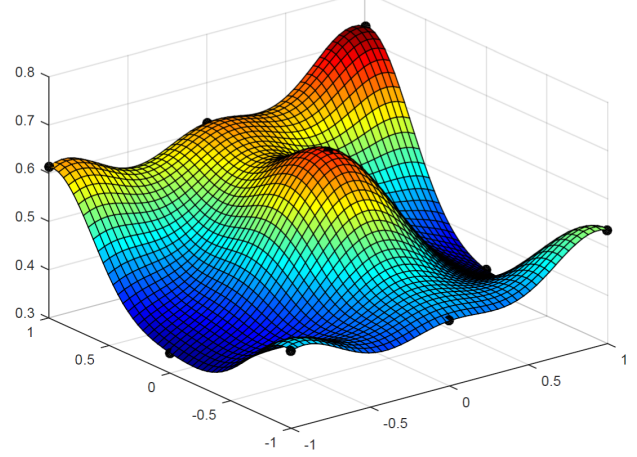

(c) $\varepsilon=0.5$

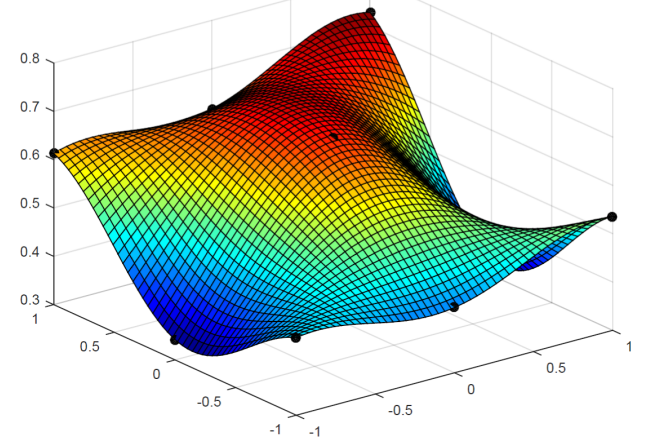

(b) $\varepsilon=0.25$

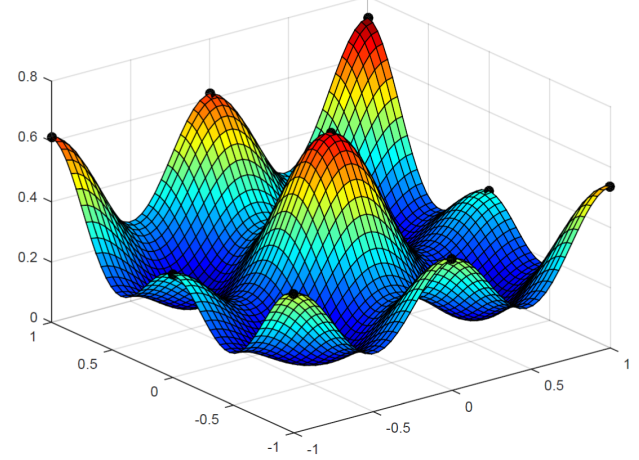

(d) $\varepsilon=0.75$

o número de condição da matriz de interpolação. A Figura 8 ilustra o efeito da variação deste parâmetro em um problema de interpolação de um conjunto de dados, utilizando $\phi(r)=e^{-\varepsilon r^{2}}$. Em Wendland (2005) podemos encontrar relações entre o parâmetro de forma e o número de condição da matriz. Já em Fasshauer (2007) encontramos algumas estratégias que podem auxiliar na escolha do parâmetro, além de indicações de trabalhos cujos autores utilizam parâmetros $\varepsilon_{j}$ diferentes para cada centro $x_{j}$. 


\subsubsection{RBFs com Suporte Compacto}

A dimensão do sistema linear, no problema de interpolação, é proporcional ao número de pontos dados. Isso significa que, em problemas de larga escala, a matriz de interpolação pode se tornar além de densa, mal condicionada e com alto custo computacional. Isto é comum ocorrer quando são utilizadas RBF's com natureza global pois, todo ponto acaba sendo influenciado por todos os centros de controle, mesmo que a RBF possua parâmetro de forma. Além disso, o controle local sobre o interpolante como nas Splines por exemplo, é fundamental em diversos problemas. Para isso, são utilizadas funções de natureza local, as funções de base radial com suporte compacto conhecidas do inglês como Compactly Supported Radial Basis Functions (CSRBF).

O suporte de uma função $\phi: \mathbb{R}^{n} \rightarrow \mathbb{R}$, denotado por $\operatorname{supp}(\phi)$, é dado pelo fecho do conjunto $\{x: \phi(x) \neq 0\}$. Se $\operatorname{supp}(\phi)$ é compacto então dizemos que $\phi$ possui suporte compacto. Atualmente, existem diversas famílias de CSRBF na literatura. Uma das mais utilizadas foi construída por Wendland (1995), denotadas por $\phi_{n, k}$, sendo funções de classe $C^{2 k}$ e definidas positivas em $\mathbb{R}^{n}$. Na Tabela 2 são apresentados alguns exemplos dessas funções, em que $(x)_{+}=x$ se $x \geq 0$ e $(x)_{+}=0$ se $x<0$.

Tabela 2 - CSRBFs de Wendland

\begin{tabular}{c|l|c}
\hline Dimensão & $\phi(r), r=\|x\|_{2}$ & Classe \\
\hline $\mathrm{d}=1$ & $\phi_{1,0}=(1-r)_{+}$ & $C^{0}$ \\
& $\phi_{1,1}=(1-r)_{+}^{3}(3 r+1)$ & $C^{2}$ \\
& $\phi_{1,2}=(1-r)_{+}^{5}\left(8 r^{2}+5 r+1\right)$ & $C^{4}$ \\
\hline $\mathrm{d}=2,3$ & $\phi_{3,0}=(1-r)_{+}^{2}$ & $C^{0}$ \\
& $\phi_{3,1}=(1-r)_{+}^{4}(4 r+1)$ & $C^{2}$ \\
& $\phi_{3,2}=(1-r)_{+}^{6}\left(35 r^{2}+18 r+3\right)$ & $C^{4}$ \\
& $\phi_{3,3}=(1-r)_{+}^{8}\left(32 r^{3}+25 r^{2}+8 r+1\right)$ & $C^{6}$ \\
\hline
\end{tabular}

Nas CSRBF's, o parâmetro de forma $\varepsilon$ controla exatamente o tamanho do suporte da função. As funções da Tabela 2 possuem suporte no intervalo $[0,1]$ e para escalar esse intervalo basta fazer $\phi_{\varepsilon}(r)=\phi(\varepsilon r)$. Com isso, $\phi_{\varepsilon}$ terá um suporte com raio $\rho=1 / \varepsilon$, ou seja, $\phi_{\varepsilon}(r)=0$ para $r>\rho$. Neste trabalho, são utilizadas apenas as CSRBF's de Wendland, em particular a $\phi_{3,3}$ pois, é compatível com problemas bidimensionais e tridimensionais e, possuir suficiente diferenciabilidade. Boas referências em RBFs são, por exemplo, Wendland (2005) e Fasshauer (2007).

\subsection{Interpolação via Núcleos Matriciais}

Os dados de um problema de interpolação não são limitados apenas a grandezas escalares. De fato, muitas aplicações de fenômenos físicos, como simulação de fluídos, envolvem dados vetoriais. Durante décadas, a reconstrução de um campo vetorial era obtida através da aproxi- 
mação/interpolação de cada componente do campo, de forma independende. Porém, muitos desses campos possuem propriedades intrínsecas, naturais do problema, que de certa forma estão relacionadas com as próprias componentes do campo, tornando praticamente impossível de se obter bons resultados, que venham a reproduzir tais propriedades. Logo, existe a necessidade de se construir interpolantes para funções vetoriais compatíveis com propriedades específicas do campo, em particular a divergência nula em todo o domínio. Nesta seção, é abordado o problema de interpolação de dados vetoriais de forma análoga ao caso escalar, através de funções positivas definidas, para construir interpolantes de funções vetoriais com a propriedade de divergência nula.

\subsubsection{Interpolação com Núcleos Matriciais de Funções de Base Ra- dial}

Semelhante ao caso escalar, estamos interessados e determinar um interpolante para os valores $f_{1}, \ldots, f_{N} \in \mathbb{R}^{d}$ amostrados nos pontos distintos $X=\left\{x_{1}, \ldots, x_{N}\right\} \subseteq \mathbb{R}^{d}$ de um campo vetorial $f: \mathbb{R}^{d} \rightarrow \mathbb{R}^{d}$ desconhecido. De forma análoga, o interpolande é construído através de combinações lineares de funções de base porém, funções matriciais $\Phi: \mathbb{R}^{d} \rightarrow \mathbb{R}^{d \times d}$ construidas através de certos operadores lineares $\mathscr{L}$ aplicados em RBFs do tipo $\phi\left(\left\|\cdot-x_{j}\right\|\right)$, ou seja, são núcleos matriciais $\Phi(x):=(\mathscr{L} \phi)(x)$, conhecidas como Matrix Valued Radial Basis Function (MVRBF). Assim, a função interpoladora possui a forma:

$$
s_{f}=\sum_{j=1}^{N} \Phi\left(\cdot-x_{j}\right) c_{j}
$$

com os coeficientes vetoriais $\left\{c_{j}\right\}_{j=1}^{N} \subset \mathbb{R}^{d}$. Tais coeficientes são obtidos impondo as condições de interpolação $s_{f}\left(x_{j}\right)=f_{j}$, resultando no sistema linear $A c=f$ :

$$
\underbrace{\left[\begin{array}{cccc}
\Phi\left(x_{1}-x_{1}\right) & \Phi\left(x_{1}-x_{2}\right) & \cdots & \Phi\left(x_{1}-x_{N}\right) \\
\Phi\left(x_{2}-x_{1}\right) & \Phi\left(x_{2}-x_{2}\right) & \cdots & \Phi\left(x_{2}-x_{N}\right) \\
\vdots & \vdots & \ddots & \vdots \\
\Phi\left(x_{N}-x_{1}\right) & \Phi\left(x_{N}-x_{2}\right) & \cdots & \Phi\left(x_{N}-x_{N}\right)
\end{array}\right]}_{A} \underbrace{\left[\begin{array}{c}
c_{1} \\
c_{2} \\
\vdots \\
c_{N}
\end{array}\right]}_{c}=\underbrace{\left[\begin{array}{c}
f_{1} \\
f_{2} \\
\vdots \\
f_{N}
\end{array}\right]}_{f} .
$$

A matriz $A$ acima, possui dimensão $N d \times N d$ pois, cada elemento $A_{i, j}=\Phi\left(x_{i}-x_{j}\right)$ é dado por um bloco de dimensão $d \times d$, enquanto $c$ e $d$ são ambos vetores com dimensão $N d \times 1$. Um exemplo trivial para o operador $\mathscr{L}$, é considerar $\Phi:=I \phi$, onde $I_{d \times d}$ é a matriz identidade. Neste caso, trata-se de interpolação componente a componente do campo, com a seguinte forma 
explícita do interpolante

$$
\begin{aligned}
s_{f} & =\sum_{j=1}^{N} I \phi_{j}(\cdot) c_{j} \\
& =\left[\begin{array}{c}
\sum_{j=1}^{N} I \phi_{j}(\cdot) c_{j}^{x} \\
\sum_{j=1}^{N} I \phi_{j}(\cdot) c_{j}^{y}
\end{array}\right] .
\end{aligned}
$$

A construção de núcleos MVRBF não é uma tarefa simples pois, a positividade de $\Phi$ também é fundamental para a existência e unicidade do interpolante. De forma geral, uma função $\Phi: \mathbb{R}^{d} \rightarrow \mathbb{R}^{n \times n}$ é dita ser definida positiva se for par $\Phi(-x)=\Phi(x)$, simétrica $\Phi(x)=\Phi(x)^{\top} \mathrm{e}$ satisfazer

$$
\sum_{j, k=1}^{N} \alpha_{j}^{\top} \Phi\left(x_{j}-x_{k}\right) \alpha_{k}>0,
$$

para todo par distinto $x_{j} \in \mathbb{R}^{d}$ e todo $\alpha_{j} \in \mathbb{R}^{n}$ tal que nem todos sejam nulos. Desta forma, a matriz de interpolação $A$ será simétrica e positiva definida, e então o Sistema (3.4) terá solução única. Na seção a seguir, é apresentado dois núcleos MVRBF que produzem interpolantes com divergência nula e rotacional nulo respectivamente.

\subsubsection{Núcleos MVRBF com Divergente Nulo e Rotacional Nulo}

Narcowich e Ward (1994) introduziram os alicerces para a construção de núcleos MVRBF no contexto de interpolação generalizada. Em particular, apresentaram um núcleo que produz interpolantes com divergente nulo em todo o domínio. Este núcleo é obtido, simplesmente, aplicando o operador $\mathscr{L}=\left(-\Delta I+\nabla \nabla^{\top}\right)$ em alguma $\operatorname{RBF} \phi\left(\| \cdot-x_{j}||\right)$, com certa regularidade, ou seja,

$$
\Phi_{d i v}:=\left(-\Delta I+\nabla \nabla^{\top}\right) \phi,
$$

em que $\nabla$ é o operador gradiente e $\Delta$ é o operador Laplaciano.

O motivo pelo qual o núcleo $\Phi_{d i v}$ produz funções com divergente nulo, na combinação linear (3.3), é o fato das colunas de $\Phi_{d i v}$ terem divergente nulo. De fato, seja $e_{j}$ o vetor da base canônica com o j-ésimo elemento igual a 1 então, a j-ésima coluna de $\Phi_{d i v}$ é dada por

$$
\begin{aligned}
\Phi_{d i v} e_{j} & =\left[\left(-\Delta I+\nabla \nabla^{\top}\right) \phi\right] e_{j} \\
& =-(\Delta I \phi) e_{j}+\left(\nabla \nabla^{\top} \phi\right) e_{j} \\
& =-\Delta\left(\phi e_{j}\right)+\nabla\left[\nabla \cdot\left(\phi e_{j}\right)\right] .
\end{aligned}
$$

Pela a identidade do Laplaciano vetorial $\nabla(\nabla \cdot F)-\nabla \times(\nabla \times F)=\Delta F$, temos que $\Phi_{d i v} e_{j}=$ $\nabla \times\left[\nabla \times\left(\phi e_{j}\right)\right]$ é dado pelo rotacional de um campo vetorial e portanto $\nabla \cdot \Phi_{d i v} e_{j}=0$. Como o divergente é um operador linear, a propriedade se mantém com a combinação linear do interpolante.

O trabalho duro feito em Narcowich e Ward (1994) é com respeito a positividade desse núcleo, fornecendo todo o respaldo matemático necessário e os requisitos sobre a função $\phi$ 
para que $\Phi_{d i v}$ seja definida positiva. Porém, para problemas em dimensão $d=2,3$, tal como no contexto deste trabalho. Assim, basta que a função $\phi$ escolhida seja definida positiva e de classe $C^{2}$.

Do ponto de vista prático, núcleo $\Phi_{d i v}$ é um a matriz de dimensão $d \times d$ e assim, precisamos explicitar cada elemento $\left[\Phi_{d i v}\right]_{i, j}$. Para isso, seja $x_{i}$ o i-ésimo componente de $x \in \mathbb{R}^{d}$ e $r=\|x\|_{2}$. Aplicando os operadores da Equação 3.5 temos:

$$
\left[\Phi_{d i v}\right]_{i, j}= \begin{cases}-\sum_{\substack{k=1 \\ k \neq i}}^{d} \frac{\partial^{2}}{\partial x_{k}^{2}} \phi(r) & i=j \\ \frac{\partial^{2}}{\partial x_{i} \partial x_{j}} \phi(r) & i \neq j .\end{cases}
$$

Embora as funções de base $\phi$ mais utilizadas sejam de classe $C^{\infty}$, a norma euclidiana possui problema de diferenciabilidade na origem (quando $r=0$ ) e precisamos calcular

$$
\frac{\partial}{\partial x_{i}} \phi(r)=x_{i} \frac{1}{r} \frac{d}{d r} \phi(r)
$$

Entretanto, a singularidade da distância euclidiana pode ser removida naturalmente pelas derivadas das funções de base. Várias RBFs, em particular as de Wendland, ao deriva-las podemos escrever $\frac{d}{d r} \phi(r)=r \tilde{\phi}(r)$, motivando o uso do seguinte operador: $\mathscr{D}_{\phi}(r)=\frac{1}{r} \frac{d}{d r} \phi(r) \mathrm{e}$ $\mathscr{D}_{\phi}^{2}(r)=\frac{1}{r} \frac{d}{d r} \mathscr{D}_{\phi}(r)$. Assim obtemos as derivadas parciais de segunda ordem,

$$
\begin{aligned}
\frac{\partial^{2}}{\partial x_{i}^{2}} \phi(r) & =x_{i}\left(\frac{\partial}{\partial x_{i}} \mathscr{D}_{\phi}(r)\right)+\mathscr{D}_{\phi}(r) \\
& =x_{i} x_{i} \frac{1}{r} \frac{d}{d r} \mathscr{D}_{\phi}(r)+\mathscr{D}_{\phi}(r) \\
& =x_{i}^{2} \mathscr{D}_{\phi}^{2}(r)+\mathscr{D}_{\phi}(r)
\end{aligned}
$$

$\mathrm{e}$

$$
\begin{aligned}
\frac{\partial^{2}}{\partial x_{i} \partial x_{j}} \phi(r) & =x_{i}\left(\frac{\partial}{\partial x_{j}} \mathscr{D}_{\phi}(r)\right) \\
& =x_{i} x_{j} \frac{1}{r} \frac{d}{d r} \mathscr{D}_{\phi}(r) \\
& =x_{i} x_{j} \mathscr{D}_{\phi}^{2}(r) .
\end{aligned}
$$

Portanto, substituindo 3.7 e 3.8 na Equação 3.6 temos:

$$
\left[\Phi_{d i v}\right]_{i, j}= \begin{cases}-\sum_{\substack{k=1 \\ k \neq i}}^{d} x_{k}^{2} \mathscr{D}_{\phi}^{2}(r)-(d-1) \mathscr{D}_{\phi}(r) & i=j \\ x_{i} x_{j} \mathscr{D}_{\phi}^{2}(r) & i \neq j .\end{cases}
$$

Em particular, para $\mathrm{d}=2$, temos o núclueo matricial

$$
\Phi_{d i v}(r)=\left[\begin{array}{cc}
-y^{2} \mathscr{D}_{\phi}^{2}(r)-\mathscr{D}_{\phi}(r) & x y \mathscr{D}_{\phi}^{2}(r) \\
x y \mathscr{D}_{\phi}^{2}(r) & -x^{2} \mathscr{D}_{\phi}^{2}(r)-\mathscr{D}_{\phi}(r)
\end{array}\right] .
$$


Figura 9 - Exempolo bidimensional de $\Phi_{d i v} \operatorname{com} \phi_{3,3}$

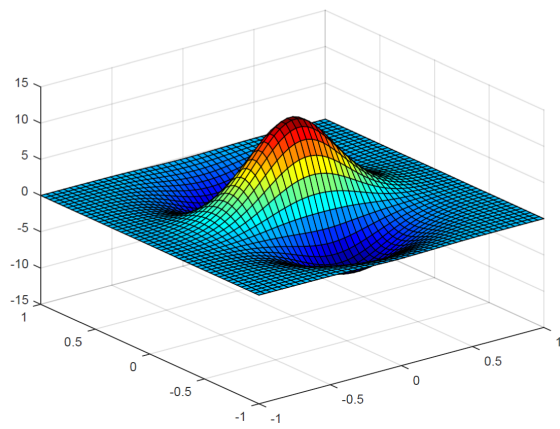

(a) $\left[\Phi_{d i v}\right]_{1,1}$

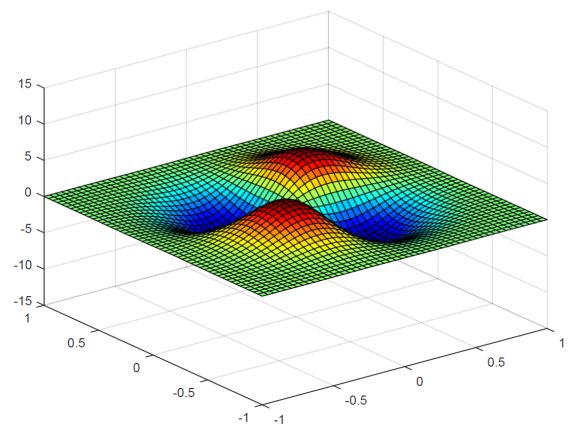

(c) $\left[\Phi_{d i v}\right]_{2,1}$

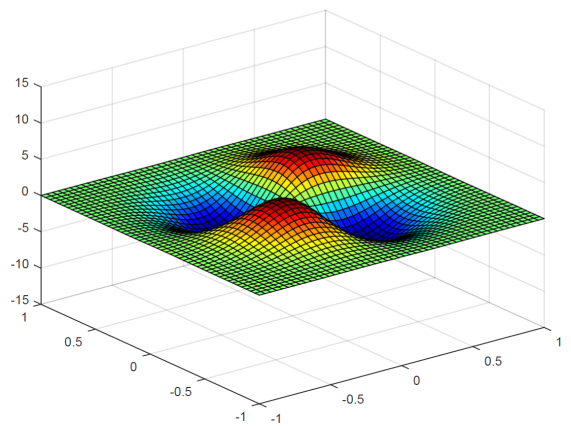

(b) $\left[\Phi_{d i v}\right]_{1,2}$

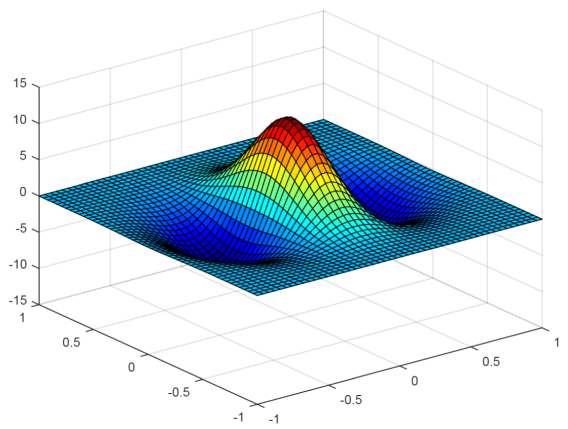

(d) $\left[\Phi_{\text {div }}\right]_{2,2}$

Na Figura 9, podemos observar os elementos independentes do núcleo Equação 3.9) utilizando a função de Wendland $\phi_{3,3}(r)=(1-\varepsilon r)_{+}^{8}\left(32 \varepsilon r^{3}+25 \varepsilon r^{2}+8 \varepsilon r+1\right) \operatorname{com} \varepsilon=0.75$.

Semelhante ao núcleo anterior, Fuselier (2006) apresentou o núcleo matricial MVRBF de rotacional nulo $\Phi_{\text {curl }}$. E de forma análoga, este núcleo também é produzido de forma muito simples, basta tomar o operador diferencial $\mathscr{L}=-\nabla \nabla^{\top}$ e aplica-lo a uma RBF positiva definida $\phi \in C^{2}$ :

$$
\Phi_{\text {curl }}:=\left(-\nabla \nabla^{\top}\right) \phi
$$

em que $\nabla$ é o operador gradiente.

Assim como o núcleo anterior, $\Phi_{\text {curl }}$ produz funções com rotacional nulo pois, suas colunas são rotacional nulo. De fato, seja $e_{j}$ o vetor da base canônica com o j-ésimo elemento igual a 1 então, a j-ésima coluna de $\Phi_{\text {curl }}$ é dada por

$$
\begin{aligned}
\Phi_{c u r l} e_{j} & =\left[\left(-\nabla \nabla^{\top}\right) \phi\right] e_{j} \\
& =\nabla\left[\left(-\nabla^{\top} \phi\right) e_{j}\right] \\
& =\nabla[\underbrace{\nabla \cdot\left(-\phi e_{j}\right)}_{g}] \\
& =\nabla g .
\end{aligned}
$$

em que $g=-\frac{\partial \phi}{\partial x_{j}}$, ou seja, $\Phi_{c u r l} e_{j}$ é o gradiente de uma função escalar portanto, $\nabla \times \Phi_{\text {curl }} e_{j}=0$. 
Figura 10 - Interpolações utilizando os núcleos $\Phi_{d i v}$ e $\Phi_{\text {curl }}$.

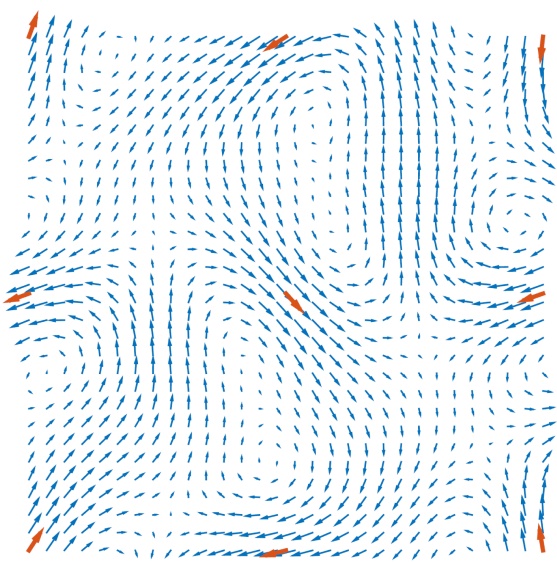

(a) Campo com divergência nula

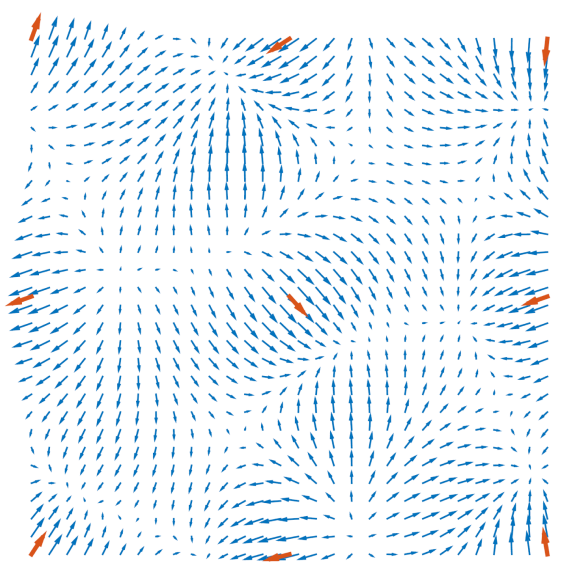

(b) Campo com rotacional nulo

Explicitando os elementos da Equação 3.10 temos:

$$
\left[\Phi_{\text {curl }}\right]_{i, j}= \begin{cases}-\frac{\partial^{2}}{\partial x_{k}^{2}} \phi(r) & i=j \\ -\frac{\partial^{2}}{\partial x_{i} \partial x_{j}} \phi(r) & i \neq j .\end{cases}
$$

Portanto, substituindo 3.7 e 3.8 na Equação 3.11 temos:

$$
\left[\Phi_{\text {curl }}\right]_{i, j}= \begin{cases}-x_{i}^{2} \mathscr{D}_{\phi}^{2}(r)-\mathscr{D}_{\phi}(r) & i=j \\ -x_{i} x_{j} \mathscr{D}_{\phi}^{2}(r) & i \neq j .\end{cases}
$$

Em Lowitzsch (2005) e Fuselier (2006), podemos encontrar estimativas de estabilidade e erro. Wendland (2009) utilizou o núcleo $\Phi_{d i v}$ para desenvolver uma discretização, sem malha, para as equações de Stokes, obtendo aproximações de alta ordem, com os erros analizados em espaços de Sobolev. Já Fuselier e Wright (2017), utilizaram ambos os núcleos para construir um método para calcular a decomposição de Helmholtz-Hodge. A Figura 10 ilustra uma simples interpolação vetorial utilizando os núcleos $\Phi_{d i v}$ e $\Phi_{c u r l}$, obtendo analiticamente campos vetoriais com divergente nulo e rotacional nulo, respectivamente.

\subsection{Métrica Restrita $\times$ Métrica Intrinseca}

Como visto anteriormente, mesmo em domínios arbitrários $X \subset \mathbb{R}^{n}$, as RBFs dependem exclusivamente da distância euclidiana entre pontos. Desta forma, o interpolante não considera informações geométricas e topológicas de domínio pois, estamos utilizando a mesma métrica euclidiana do $\mathbb{R}^{2}$ apenas restrita ao domínio. Essa tipo de métrica é conhecida por métrica restrita e é denotada por $d_{\mathbb{R}^{2} \mid X}$. Em domínios não convexos, as métricas restritas podem não condizer com noções geométricas intuitivas, por exemplo, vizinhança de um ponto. Utilizar tais informações geométricas podem trazer melhorias na precisão e estabilidade do interpolante. 
Figura 11 - Mapa de distâncias, a partir de um ponto, representado pela escala de cores.

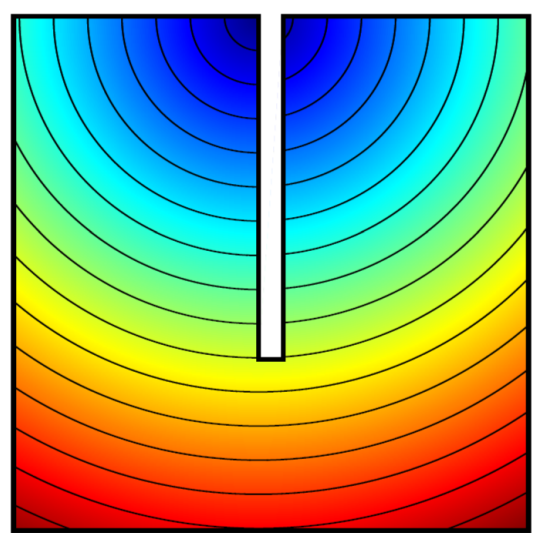

(a) Métrica Restrita

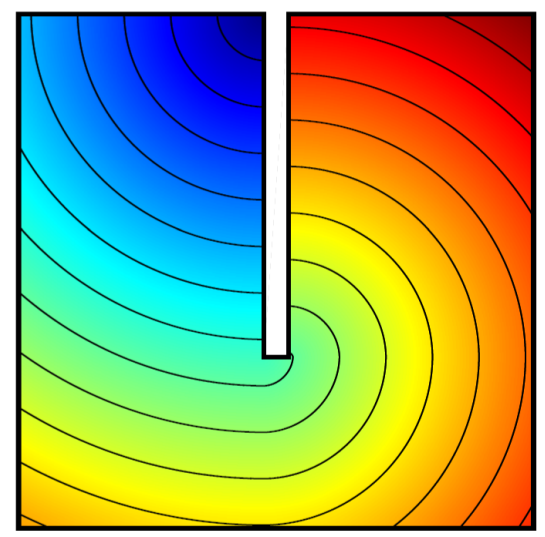

(b) Métrica Intrinseca

Nessa seção, é feita uma breve discução sobre o um problema do uso de métricas restritas com funções de base radial e uma possível alternativa com o uso de métricas intrínsecas, apresentadas a seguir. Na Figura 11, temos uma comparação entre estas duas métrica, ilustrando o mapa de distâncias a um ponto. No contexto computacional, Bronstein, Bronstein e Kimmel (2008) e Peyré et al. (2010) são boas referências do assunto.

\subsubsection{Métricas Intrínsecas e Funções de Bases Radiais}

No caso de interpolação em domínios não convexos, temos um problema da natureza geométrica associada com a região de influência da RBF. Basicamente, a vizinhança dada pela distância euclidiana não condiz com a vizinhança intrinseca do domínio e com isso, pontos equivocados podem ser considerados pelas funções de base. Por exemplo, seja o domínio $\Omega \subset \mathbb{R}^{2}$ da Figura 12 (a) e uma discretização $X \subset \mathbb{R}^{2}$ em (b). A circunferência tracejada determina a vizinhança do ponto central, pontos vermelhos, sendo exatamente a região determinada pelo suporte de alguma função.

Figura 12 - Vizinhança em $\mathbb{R}^{2}$

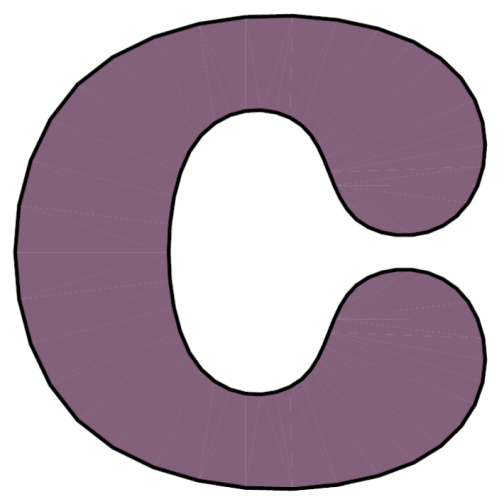

(a) Domínio $\Omega \subset \mathbb{R}^{2}$

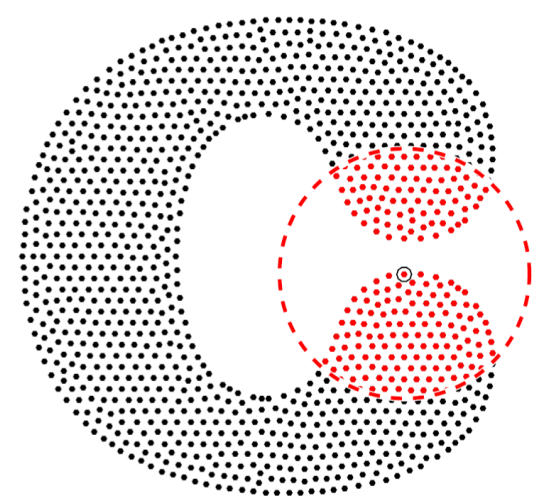

(b) Discretização $X \subset \mathbb{R}^{2}$ 
Uma forma de considerar as propriedades geométricas e topológicas do domínio é definir uma métrica em $X$ associada a uma estrutura de comprimento. Esta estrutura consiste numa classe de caminhos admissíveis, suaves por partes e contidos em $X$, para os quais podemos medir seus comprimentos, através de um funcional $L$, correspondendo a cada caminho da classe a um número real não negativo. Essa métrica, associada a esta estrutura, é conhecida como métrica intrinseca. Mais precisamente, com uma estrutura de comprimento, associamos para dois pontos $x, y \in X$ a distância entre eles como sendo o ínfimo do comprimento dos caminhos admissíveis entre esses pontos (o menor caminho pode não existir) assim, seja $\mathscr{C}$ a classe de todos os caminhos admissíveis $\gamma:[a, b] \rightarrow X$ tais que $\gamma(a)=x$ e $\gamma(b)=y$ então

$$
d_{L}(x, y)=\inf _{\gamma \in \mathscr{C}}\{L(\gamma)\} .
$$

No contexto de funções de bases radiais, o uso de métricas intrinsecas é quase inexistente. Em Wendland (2005), são introduzidos os conceitos básicos da teoria de interpolação de dados esparsos sobre variedades compactas, com exemplo concreto na esfera. Já Narcowich, Ward e Wright (2007) traz o núcleo de divergente nulo para domínios esféricos utilizando uma dada distância na esfera. Recentemente, Levi e Levin (2014), utilizam RBFs para controlar a deformação de objetos tridimensionais, substituindo diretamente a distância euclidiana por uma distância definida no interior do objeto. A seguir, apresentamos as duas distâncias interiores utilizadas neste trabalho.

\subsubsection{Distância Geodésica Discreta via FMM}

Do ponto de vista computacional, podemos aproximar uma métrica intrinseca através da representação em grafo de uma discretização do domínio $X$. Assim, o problema é reduzido à determinação do menor caminho no grafo. Para isso temos o algoritmo de Dijkstra como uma ótima opção porém, dependendo da conectividade dos vizinhos, a métrica intrinseca obtida pode ser inconsistente com a euclidiana. Em um grid, por exemplo, é calculada a distância $\ell^{1}$ (distância de Manhattan) pois, não são considerados os caminhos que cruzam as arestas do grafo. Sethian (1999) introduziu uma família de algoritmos chamados, do inglês, de Fast Marching Method (FMM), que basicamente, permite caminhos entre as arestas, calculando consistentemente distâncias em grids e malhas triângulares. Mais precisamente, o FMM, é um método numérico muito eficiente para resolver a equação diferencial parcial não linear Eikonal:

$$
\begin{aligned}
\|\nabla U(x)\| & =1, \quad x \in X \\
U\left(x_{0}\right) & =0,
\end{aligned}
$$

cuja solução, para cada ponto $x_{0} \in X$ fixado, é o mapa de distâncias

$$
U_{x_{0}}(x)=d_{L}\left(x_{0}, x\right) .
$$

$\mathrm{Na}$ equação diferencial acima, também podemos considerar a condição de contorno $U(x)=0, \quad x \in \partial X$ ao invés do valor inicial e assim, obtemos o mapa de distâncias da fronteira 
$U_{\partial X}(x)=\min _{y \in \partial X} U_{x}(y)$ como solução da equação. Na Figura 13 temos a malha triangular de um domínio ilustrando estas duas opções da equação diferencial, obtendo como solução o mapa de distâncias a partir de um ponto e o mapa de distâncias do contorno.

Figura 13 - Soluções da equação Eikonal através do método FMM. A escala de cores corresponde as distâncias.

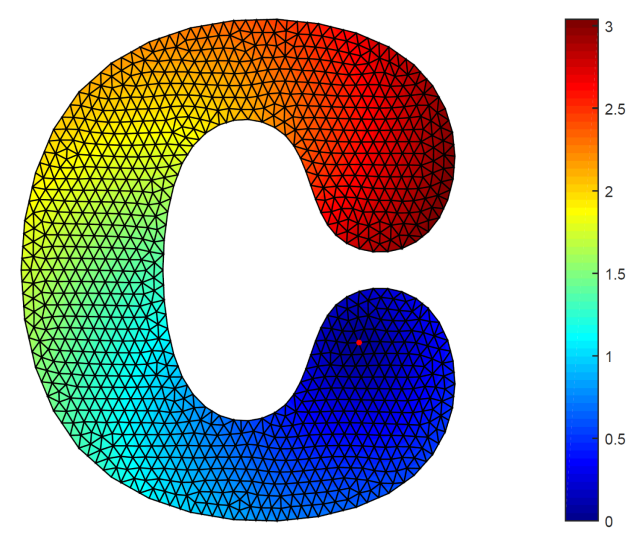

(a) Mapa de distância a partir de um ponto

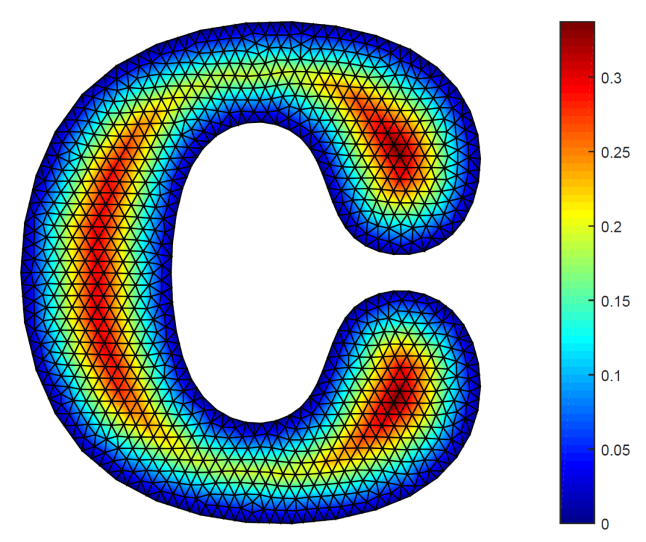

(b) Distância da fronteira

\subsubsection{Distância Interior usando Coordenadas Baricêntricas}

Rustamov, Lipman e Funkhouser (2009) apresentam uma forma elegante e eficiente de definir uma distância entre dois pontos no interior de uma região poligonal fechada (curva poligonal ou malha triangular) propagando-se para o interior as distâncias entre os vértices. Especificamente, dada uma malha com vértices $v_{i} \in \mathbb{R}^{3}, \quad i=1, \ldots, n$ e o conjunto de distâncias $d_{i j}=d\left(v_{i}, v_{j}\right)$ sobre a malha. A primeira etapa consiste em construir um mapa $v_{i} \longmapsto v_{i}^{*} \in \mathbb{R}^{m}$ que realiza uma imersão dos vértices $v_{i} \in \mathbb{R}^{3}$ em $\mathbb{R}^{m}$ para algum $m$ tal que $\left\|v_{i}^{*}-v_{j}^{*}\right\|_{\mathbb{R}^{m}}$ seja igual ou aproximadamente $d_{i j}$. Os autores destacam a utilização da imersão dada pelo mapa de difusão, utilizado para obter a distância de difusão. Assim, seja $\left\{\lambda_{k}, \phi_{k}\right\}_{k=1}^{m}$ a decomposição do operador discreto de Laplace-Beltrami associado a malha. O mapa de difusão é dado pela imersão:

$$
v_{i} \longmapsto\left(e^{-\lambda_{1} t} \phi_{1}\left(v_{i}\right), e^{-\lambda_{2} t} \phi_{2}\left(v_{i}\right), \ldots, e^{-\lambda_{m} t} \phi_{m}\left(v_{i}\right)\right) \in \mathbb{R}^{m}
$$

em que $\phi_{k}\left(v_{i}\right)$ é a i-ésima entrada do vetor $\phi_{k}$. No trabalho citado, podemos encontar todas as tecnicalidades desta imersão assim como referências a respeito da decomposição do operador de Laplace-Beltrami e outras formas de obter a imersão dos vértices.

A segunda etapa consiste em estender o mapa de imersão para pontos do interior da malha através de interpolação baricentrica, fazendo:

$$
p \longmapsto p^{*}=\sum_{i} w_{i}(p) v_{i}^{*}
$$


em que $w_{i}(\cdot)$ são as coordenadas baricentricas referente ao vértice $v_{i}$. E finalmente, a distância interior entre $p$ e $q$ é definida como a distância euclidiana entre as imagens $p^{*}$ e $q^{*}$ em $\mathbb{R}^{m}$,

$$
\hat{d}(p, q):=\left\|p^{*}-q^{*}\right\|_{\mathbb{R}^{m}}
$$

Através dessa abordagem temos uma expressão analítica para a distância interior, apenas em função das coordenadas baricentricas e sem o uso de malhas. Na Figura 14 podemos ver um exemplo do mapa de distância.

Figura 14 - Mapa da distância interior a partir de um ponto.

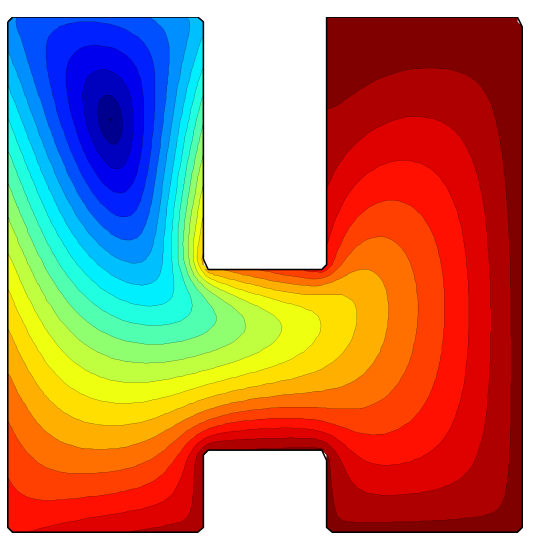

Fonte: Rustamov, Lipman e Funkhouser (2009).

Podemos obter uma expressão mais conveniente substituindo $p^{*}=\sum_{i}^{n} w_{i}(p) v_{i}^{*}$ e $q^{*}=$ $\sum_{i}^{n} w_{i}(q) v_{i}^{*}$ na Equação 3.12, assim obtemos:

$$
\begin{aligned}
\hat{d}(p, q) & =\left\langle p^{*}-q^{*}, p^{*}-q^{*}\right\rangle_{\mathbb{R}^{m}} \\
& =\left\langle\sum_{i}\left(w_{i}(p)-w_{i}(q)\right) v_{i}^{*}, \sum_{j}\left(w_{j}(p)-w_{j}(q)\right) v_{j}^{*}\right\rangle_{\mathbb{R}^{m}} \\
& =\sum_{i, j}\left(w_{i}(p)-w_{i}(q)\right)\left(w_{j}(p)-w_{j}(q)\right)\left\langle v_{i}^{*}, v_{j}^{*}\right\rangle_{\mathbb{R}^{m}},
\end{aligned}
$$

ou seja, uma forma quadrática em $w_{i}(p)-w_{i}(q)$. Denotando por $w(p)=\left(w_{1}(p), w_{2}(p), \ldots, w_{n}(p)\right)^{\top}$, obtemos a forma matricial

$$
\hat{d}(p, q)=\sqrt{\left(w_{i}(p)-w_{i}(q)\right)^{\top} A\left(w_{i}(p)-w_{i}(q)\right)},
$$

em que $A_{i, j}=\left\langle v_{i}^{*}, v_{j}^{*}\right\rangle_{\mathbb{R}^{m}}$. 

CAPÍTULO

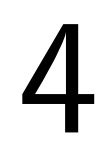

\section{INTERPOLANDO CAMPOS VETORIAIS EM SUPERFÍCIE}

Embora o design de campos vetoriais em superfícies não seja o foco deste trabalho, com uma simples aplicação das ferramentas apresentas no capítulo anterior podemos obter bons resultados. Além disso, exploramos uma possível alternativa sem malha nesta área, contrapondo com os métodos mais tradicionais da literatura.

Consideremos o seguinte problema: seja $\mathscr{P} \subset \mathbb{R}^{3}$ uma nuvem de pontos amostrados de uma superfície tridimensional juntamente com o conjunto $\mathscr{N} \subset \mathbb{R}^{3}$ de vetores normais nestes pontos. Queremos construir um campo vetorial tangente sobre esta nuvem de pontos a partir de um conjunto de vetores de controle $v_{i} \in \mathbb{R}^{3}$ localizados nos pontos $\left\{x_{i}=\left(x_{i}^{x}, x_{i}^{y}, x_{i}^{z}\right) \in \mathscr{P}, i=\right.$ $1, \ldots, N\}$. A proposta deste capítulo, é tratar esse problema como uma simples interpolação utilizando RBFs e os núcleos de divergente núlo e rotacional nulo apresentados anteriormente.

\subsection{Interpolação com RBF por Componente}

Com alguma $\mathrm{RBF} \phi: \mathbb{R}^{3} \rightarrow \mathbb{R}$, podemos interpolar componente a componente os vetores de controle, ou seja, tratar cada coordenada como uma função escalar. Desta forma, obtemos o seguinte interpolante vetorial:

$$
F=\sum_{j=1}^{N} \phi\left(\left\|\cdot-x_{j}\right\|\right) \alpha_{j}
$$

em que $\alpha_{j}=\left(\alpha_{j}^{x} \alpha_{j}^{y} \alpha_{j}^{z}\right)^{\top}$ são os coeficientes a serem determinados, assim como no caso escalar. Desta forma, temos uma combinação linear dos vetores $\alpha_{j} \in \mathbb{R}^{3}$.

Para determinar os coeficientes $\alpha_{j}$, utilizamos como dados de interpolação, as projeções de cada vetor de controle $v_{i}$ na superfície, apenas para melhorar a intuição de modelagem, para condizer com o resultado final. Esta projeção é dada através de cada vetor normal $n_{i}=$ 
$\left(n_{i}^{x}, n_{i}^{y}, n_{i}^{z}\right) \in \mathscr{N}$ associado ao ponto $x_{i}$ tomando:

$$
t_{i}=v_{i}-\operatorname{proj}_{n_{i}} v_{i}, 1 \leq i \leq N
$$

Assim, aplicando as condições de interpolação $F\left(x_{i}\right)=t_{i}, \operatorname{com} t_{i}=\left(t_{i}^{x} t_{i}^{x} t_{i}^{x}\right)$, na Equação 4.1, temos:

$$
\left[\begin{array}{c}
t_{i}^{x} \\
t_{i}^{y} \\
t_{i}^{z}
\end{array}\right]=\sum_{j=1}^{N} \phi\left(\left\|x_{i}-x_{j}\right\|\right)\left[\begin{array}{c}
\alpha_{i}^{x} \\
\alpha_{i}^{y} \\
\alpha_{i}^{z}
\end{array}\right], i=1,2, \ldots, N,
$$

ou seja, devemos resolver três sistemas lineares $A \alpha^{x}=t^{x}, A \alpha^{y}=t^{y}$ e $A \alpha^{z}=t^{z}$ em que

$$
A=\left[\begin{array}{cccc}
\phi\left(\left\|x_{1}-x_{1}\right\|\right) & \phi\left(\| x_{1}-x_{2}||\right) & \cdots & \phi\left(\| x_{1}-x_{N}||\right) \\
\phi\left(\left\|x_{2}-x_{1}\right\|\right) & \phi\left(\| x_{2}-x_{2}||\right) & \cdots & \phi\left(\left\|x_{2}-x_{N}\right\|\right) \\
\vdots & \vdots & \ddots & \vdots \\
\phi\left(\| x_{N}-x_{1}||\right) & \phi\left(\left\|x_{N}-x_{2}\right\|\right) & \cdots & \phi\left(\left\|x_{N}-x_{N}\right\|\right)
\end{array}\right] .
$$

Note que a matriz de interpolação $A$ é a mesma pra os três sistemas, portanto é possível otimizar numericamente as soluções, por exemplo, com a decomposição LU. Com os coeficientes $\alpha_{j}$, podemos avaliar o campo $F$ em todos os pontos de $\mathscr{P}$ porém, não temos garantia de tangência. Para isso, simplesmente projetamos o campo avaliado na superfície. A Figura 15 ilustra um exemplo deste método em uma nuvem de pontos bem simples. São 3242 pontos amostrados, com distribuição quasi-uniforme, da esfera $x^{2}+y^{2}+z^{2}=0.25$. Dentre estres pontos, temos 50 pontos de controle $x_{i}$ s com seus respectivos vetores de controle $v_{i}$ s aleatórios. A RBF utilizada é $\phi_{3,3}(r)=(1-r)_{+}^{8}\left(32 r^{3}+25 r^{2}+8 r+1\right)$.

Figura 15 - Interpolação vetorial, por componente, na esfera.

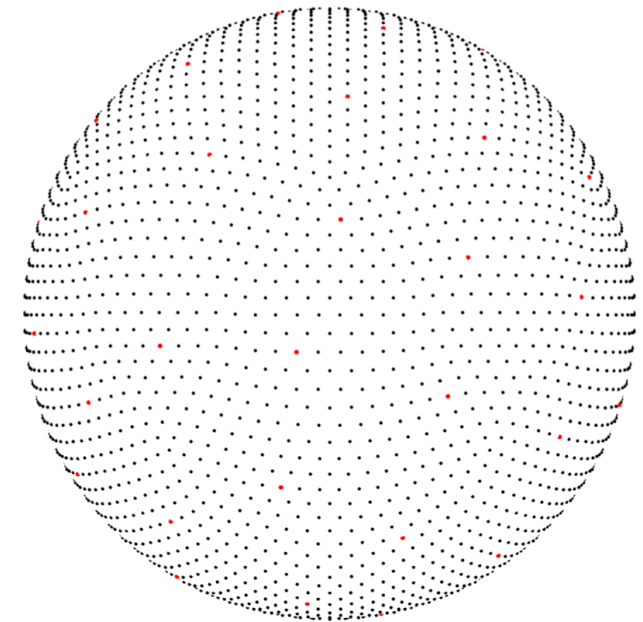

(a) Domínio discreto: pontos da esfera $x^{2}+$ $y^{2}+z^{2}=0.25$

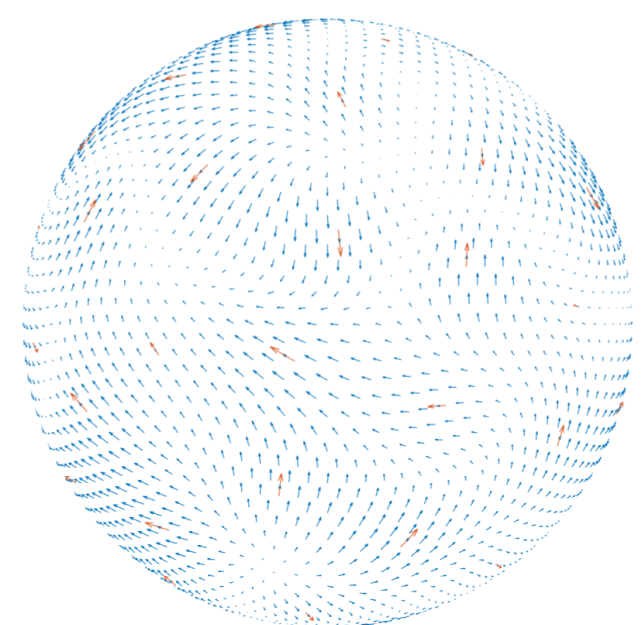

(b) Interpolação por componente dos vetores de controle em vermelho

Já na Figura 16, utilizamos uma nuvem de pontos mais complexa, com 9851 pontos, 200 pontos e vetores de controle e com a mesma RBF do exemplo anterior. 
Figura 16 - Interpolação vetorial, por componente, numa núvem de pontos do modelo bunny.

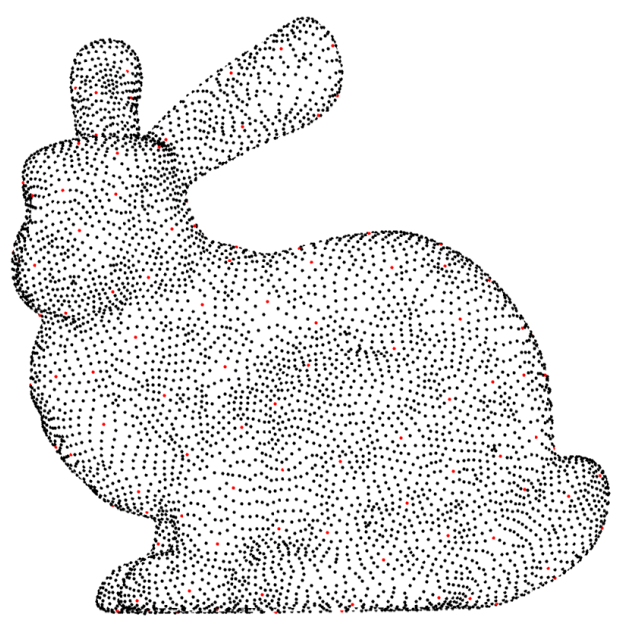

(a) Núvem de pontos e pontos de controle (vermelhos)

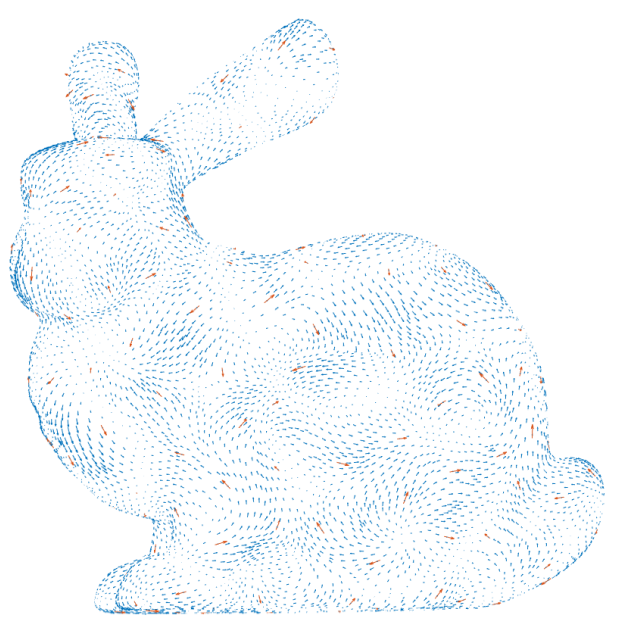

(b) Interpolação por componente dos vetores de controle em vermelho

\subsection{Interpolação com MVRBF}

Outra alternativa, para interpolar os vetores de controle, é utilizar os núcleos matricias, $\Phi_{d i v}$ e $\Phi_{c u r l}$, apresentados no capítulo anterior. Deste modo, agregamos ao campo resultante, características aproximadas desses núcleos. Assim como no método anterior, queremos interpolar as projeções dos vetores de controle, isto é, o conjunto $\left\{t_{i}\right\}_{i=1}^{N} \subset \mathbb{R}^{3}$, para obter um interpolante para os dados vetorias da forma:

$$
F=\sum_{j=1}^{N} \Phi\left(\| \cdot-x_{j}||\right) c_{j} .
$$

em que $\Phi=\Phi_{d i v}$ ou $\Phi=\Phi_{\text {curl }}$ dados a partir de alguma $\phi \in C^{2}$.

No capítulo anterior, foi apresentado apenas a versão bidimensional destes núcleos porém, com os mesmos cálculos das derivadas de segunda ordem, obtemos as versões tridimensionais:

$$
\Phi_{\text {div }}(r)=\left[\begin{array}{ccc}
-\left(y^{2}+z^{2}\right) \mathscr{D}_{\phi}^{2}(r)-2 \mathscr{D}_{\phi}(r) & x y \mathscr{D}_{\phi}^{2}(r) & x z \mathscr{D}_{\phi}^{2}(r) \\
x y \mathscr{D}_{\phi}^{2}(r) & -\left(x^{2}+z^{2}\right) \mathscr{D}_{\phi}^{2}(r)-2 \mathscr{D}_{\phi}(r) & y z \mathscr{D}_{\phi}^{2}(r) \\
x z \mathscr{D}_{\phi}^{2}(r) & y z \mathscr{D}_{\phi}^{2}(r) & -\left(x^{2}+y^{2}\right) \mathscr{D}_{\phi}^{2}(r)-2 \mathscr{D}_{\phi}(r)
\end{array}\right]
$$

$\mathrm{e}$

$$
\Phi_{\text {curl }}(r)=\left[\begin{array}{ccc}
-x^{2} \mathscr{D}_{\phi}^{2}(r)-\mathscr{D}_{\phi}(r) & -x y \mathscr{D}_{\phi}^{2}(r) & -x z \mathscr{D}_{\phi}^{2}(r) \\
-x y \mathscr{D}_{\phi}^{2}(r) & -y^{2} \mathscr{D}_{\phi}^{2}(r)-\mathscr{D}_{\phi}(r) & -y z \mathscr{D}_{\phi}^{2}(r) \\
-x z \mathscr{D}_{\phi}^{2}(r) & -y z \mathscr{D}_{\phi}^{2}(r) & -z^{2} \mathscr{D}_{\phi}^{2}(r)-\mathscr{D}_{\phi}(r)
\end{array}\right] .
$$

O restante do processo de interpolação segue análogo ao método anterior. Construímos o conjunto de equações lineares matriciais

$$
\sum_{j=1}^{N} \Phi\left(\left\|x_{i}-x_{j}\right\|\right) c_{j}=t_{i}, \quad 1 \leq i \leq N
$$


resultando no sistema linear $A c=t$, com dimensão $3 N \times 3 N$, cuja solução fornece os coeficientes $c_{j}$ s. O campo vetorial obtido possui garantia analítica de divergência nula ou rotacional nulo, entretando projetamos o campo resultante na nuvem de pontos para obter um campo tangente, perdendo estas garantias analíticas. Na Figura 17, foram utilizados os mesmos conjuntos de pontos e vetores de controle do método anterior. Nestas figuras, podemos notar características visuais dos núcleos utilizados, mesmo após as projeções desses campos na núvem de pontos.

Figura 17 - Interpolação dos vetores de controle na núvem de pontos, utilizando os núcleos $\Phi_{d i v}$ e $\Phi_{c u r l}$.

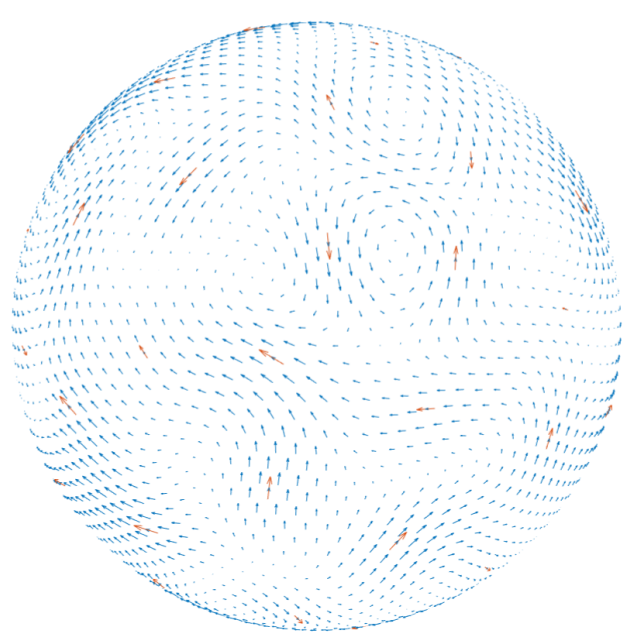

(a) Interpolação com o núcleo $\Phi_{d i v}$

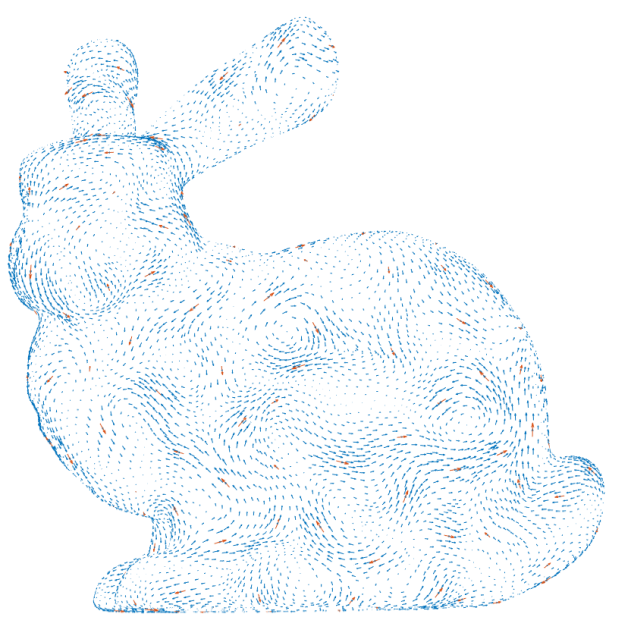

(c) Interpolação com o núcleo $\Phi_{d i v}$

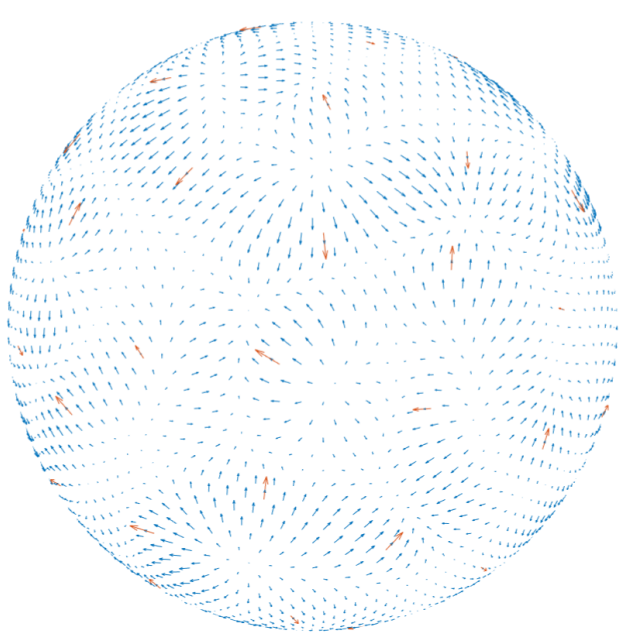

(b) Interpolação com o núcleo $\Phi_{\text {curl }}$

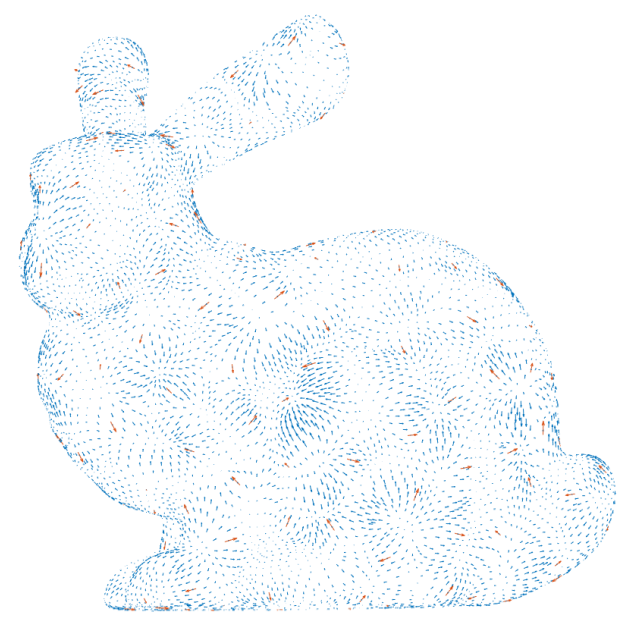

(d) Interpolação com o núcleo $\Phi_{\text {curl }}$ 


\section{ABORDAGEM PROPOSTA}

Matematicamente, o problema abordado consiste em: dado um conjunto de $N$ vetores de controle $v_{1}, \ldots, v_{N} \in \mathbb{R}^{2}$ localizados, respectivamente, nos pontos $X_{c}=\left\{x_{1}, \ldots, x_{N}\right\}$ de uma região fechada do plano, $\Omega \subset \mathbb{R}^{2}$, queremos construir um campo vetorial $F: \Omega \rightarrow \mathbb{R}^{2}$ a partir dos vetores de controle, tal que $F$ possua divergência aproximadamente nula e seja tangente a $\partial \Omega$. Para isso, consideremos como domínio discreto o conjunto de pontos $X \in \Omega$ tal que $X=X_{i} \cup X_{b}$, onde $X_{i}$ contém $N_{i}$ pontos interiores de $\Omega$ e $X_{b}$ contém os $N_{b}$ pontos da fronteira $\partial \Omega$.

O método proposto neste trabalho, é resultado de uma sequência de modificações, partindo do problema de interpolação de campos vetoriais com o núcleo MVRBF. Neste capítulo, apresentaremos o método desenvolvido, explicitando cada etapa percorrida, mostrando os problemas e dificuldades encontrados a fim de justificar as escolhas adotadas.

\subsection{Interpolação Direta via MVRBF}

Como visto anteriormente, temos na literatura um eficiente método de interpolação de campos vetorias com divergente nulo e este é nosso ponto de partida. Entretanto, a simples interpolação dos vetores de controle $v_{i}$ 's em todo o domínio $X$, não satisfaz a propriedade da tangente na fronteira $\partial \Omega$ pois, não estamos utilizando qualquer informação geométrica ou topológica de $\Omega$. Porém, podemos prosseguir com esta simples abordagem considerando dado a amostra $t_{1}, \ldots t_{N_{b}} \in \mathbb{R}^{2}$ de um campo vetorial tangente a $\partial \Omega$ nos pontos $y_{1}, \ldots, y_{N_{b}} \in X_{b}$. E então, interpolamos simultaneamente, com um único sistema linear, os vetores de controle com o campo tangente dado. Desta maneira, interpolamos os vetores de controle, ou seja, temos parâmetros de design, e forçamos o campo a tangenciar todos os pontos de avaliação da fronteira $X_{b}$. Esta é a abordagem inicial do trabalho descrita abaixo.

Concatenando os pontos de controle $X_{c}$ com os pontos da fronteira $X_{b}$, temos um novo conjunto com $M=N+N_{b}$ pontos $x_{j} \in X_{c} \cup X_{b}$ e os respectivos vetores de interpolação $f_{j} \in$ 
$\left\{v_{1}, \ldots, v_{N}, t_{1}, \ldots t_{N_{b}}\right\} \subset \mathbb{R}^{2}$. Então, aplicamos o método de interpolação apresentado na Seção 3.2.2, ou seja $\Phi_{d i v}$ o núcleo de divergente nulo, consideremos o interpolante da forma:

$$
F=\sum_{j=1}^{M} \Phi_{d i v}\left(\left\|\cdot-x_{j}\right\|\right) c_{j}
$$

Aplicando as condições de interpolação, temos as seguintes equações:

$$
\sum_{j}^{N} \Phi_{d i v}\left(\left\|x_{i}-x_{j}\right\|\right) c_{j}=f_{i}, \quad 1 \leq i \leq M
$$

resultando no sistema linear abaixo com dimensões $2 M \times 2 M$ :

$$
\left[\begin{array}{cccc}
\Phi_{1,1} & \Phi_{1,2} & \cdots & \Phi_{1, M} \\
\Phi_{2,1} & \Phi_{2,2} & \cdots & \Phi_{2, M} \\
\vdots & \vdots & \ddots & \vdots \\
\Phi_{M, 1} & \Phi_{M, 2} & \cdots & \Phi_{M, M}
\end{array}\right]\left[\begin{array}{c}
c_{1} \\
c_{2} \\
\vdots \\
c_{M}
\end{array}\right]=\left[\begin{array}{c}
f_{1} \\
f_{2} \\
\vdots \\
f_{M}
\end{array}\right]
$$

em que, cada bloco matricial $\Phi_{i, j} \in \mathbb{R}^{2} \times \mathbb{R}^{2}$ é dado por:

$$
\Phi_{i, j}=\left[\begin{array}{cc}
-y_{i j}^{2} \mathscr{D}_{\phi}^{2}\left(\left\|x_{i}-x_{j}\right\|\right)-\mathscr{D}_{\phi}\left(\left\|x_{i}-x_{j}\right\|\right) & x_{i j} y_{i j} \mathscr{D}_{\phi}^{2}\left(\left\|x_{i}-x_{j}\right\|\right) \\
x_{i j} y_{i j} \mathscr{D}_{\phi}^{2}\left(\left\|x_{i}-x_{j}\right\|\right) & -x_{i j}^{2} \mathscr{D}_{\phi}^{2}\left(\left\|x_{i}-x_{j}\right\|\right)-\mathscr{D}_{\phi}\left(\left\|x_{i}-x_{j}\right\|\right)
\end{array}\right] .
$$

A Figura 18, apresenta um exemplo da utilização do método através da implementação no software MATLAB. Utilizando o pacote de geração de malhas distmesh ${ }^{1}$, obtemos a discretização $X$, com espaçamentos quasi-uniformede, de um domínio $\Omega \subset \mathbb{R}^{2}$, ilustrado na Figura 18(a), com $|X|=1280$ pontos de avaliação, $N_{b}=193$ pontos na fronteira e $X_{c}=7$ pontos de controle. $O$ campo tangente utilizado são vetores unitários dados pela orientação anti-horária dos pontos $X_{b}$ e os vetores de controle nos pontos $X_{c}$ são unitários com direções aleatórias. Com isso, construimos o núcleo $\Phi_{d i v}$ com a CSRBF de Wendland $\phi_{3,3}(r)=(1-\varepsilon r)_{+}^{8}\left[32(\varepsilon r)^{3}+\right.$ $\left.25(\varepsilon r)^{2}+8 \varepsilon r+1\right]$, considerando $\varepsilon=1.0$ e as derivadas

$$
\begin{aligned}
& \text { - } \left.\mathscr{D}_{\phi}(r)=-22 \varepsilon^{2}(1-\varepsilon r)_{+}^{7}\left[16(\varepsilon r)^{2}+7 \varepsilon r+1\right)\right] \\
& \text { - } \mathscr{D}_{\phi}^{2}(r)=528 \varepsilon^{4}\left[(1-\varepsilon r)_{+}^{6}(6 \varepsilon r+1)\right]
\end{aligned}
$$

Na Figura 18(b) temos o resultado da interpolação dos vetores de controle (vermelhos no interior) e vetores tangentes no contorno. O campo tangente dado influencia o interpolante porém, sua finalidade é forçar o campo resultante tangenciar a fronteira e para isso, devemos ter um grande número de amostras, aumentando consideravelmente a dimensão do sistema linear, enquanto que em situações práticas, o número de vetores de controle é muito menor ou seja, temos um custo computacional elevado concentrado apenas para simular a restrição do domínio. 
Figura 18 - Interpolação simultânea dos vetores de controle no interior e do campo tangente no contorno

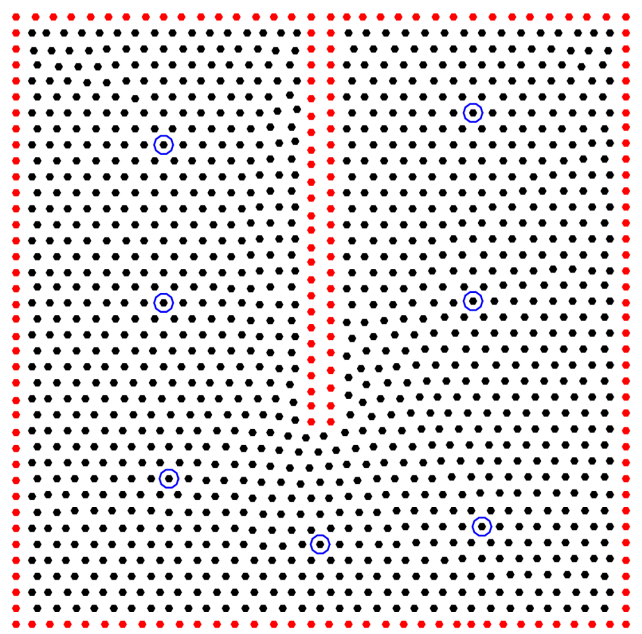

(a) Domínio discreto $X$

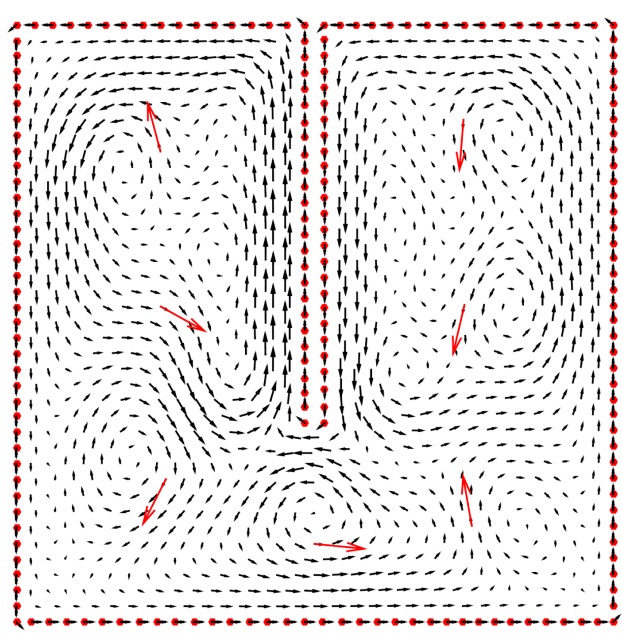

(b) Campo resultante

Fonte: Elaborada pelo autor.

Entretanto, a densidade desses pontos leva a um problema maior: a instabilidade da interpolação. A estabilidade em um problema de interpolação, é medido através do número de condição da matriz de interpolação e dizemos que quanto menor este número o problema será melhor condicionado. Lowitzsch (2002) mostrou que para o núcleo $\Phi_{\text {div }}$ com as CSRBF de Wendland, o número de condição é invesamente proporcional a distância de separação dos pontos dada por $2 q:=\min _{j \neq k}||\left|x_{j}-x_{k}\right|$ assim, com um conjunto denso de dados teremos um problema mal condicionado. Além disso, toda vez que um vetor de controle for modificado, é preciso resolver novamente o sistema.

\subsection{Método Proposto}

Para contornar os problemas do método anterior, vamos tratar de forma independente os vetores de controle e as amostras tangentes na fronteira. Nossa proposta é considerar o campo final como a soma ponderada de dois campos: $F_{c t r}: X \rightarrow \mathbb{R}^{2}$ e $F_{t a n}: X \rightarrow \mathbb{R}^{2}$ obtidos, respectivamente, através dos vetores de controle e dos vetores tangentes, tal que o campo final seja da forma:

$$
F=w_{c t r} F_{c t r}+w_{t a n} F_{t a n}
$$

em que $w_{c t r}, w_{t a n}: X \rightarrow \mathbb{R}$ são funções peso.

O campo $F_{c t r}$ carrega as informações dos vetores de controle, enquanto que $F_{\text {tan }}$ força a tangencia de $F$ nos pontos $X_{b}$ e com as funções de peso, $w_{c t r}$ e $w_{t a n}$, controlamos a influência de cada campo a fim de ajustar a suavidade da soma. Desta forma, lidamos com os dados de tangência apenas uma vez. Para obter o campo $F_{c t r}$, interpolamos os dados $\left\{x_{i}, v_{i}\right\}_{i=1}^{N} \subset \mathbb{R}^{2} \times \mathbb{R}^{2}$ em todos os pontos de $X$ com o núcleo matricial $\Phi_{d i v}$ como anteriormente, obtendo o interpolante 
com divergente nulo:

$$
F_{c t r}=\sum_{j=1}^{N} \Phi_{d i v}\left(\left\|\cdot-x_{j}\right\|\right) c_{j}
$$

A próxima etapa consiste na construção do campo tangente $F_{\text {tan }}$ em todo o conjunto $X$. Na seção anterior, exigimos um campo tangente, como restrição no sistema de interpolação. Com esta nova abordagem, não é mais necessário requerer esse campo de entrada pois, construimos uma amostra tangente utilizando o próprio $F_{c t r}$ tomando a projeção de cada $F_{c t r}\left(y_{i}\right)$ na fronteira $\partial \Omega$, obtendo o campo tangente $t_{i} \in \mathbb{R}^{2}$. Assim, podemos obter o campo $F_{\text {tan }}$ interpolando os dados $\left\{y_{i}, t_{i}\right\}_{i=1}^{N} \subset \mathbb{R}^{2} \times \mathbb{R}^{2}$ em todos os pontos de $X$ porém, ainda estaremos resolvendo um sistema relativamente grande, mesmo que uma vez. Desta forma, propomos aproximar o campo $F_{\text {tan }}$ através do processo que é conhecido na literatura por quasi-interpolação baseado em RBF (Liu e Wang (2012), Han e Hou (2008)). Este método consiste em aproximar uma função $f$ por uma média dos valores conhecidos $f_{i}$ nos pontos $x_{i}$, ponderados por RBF, isto é, $f \approx \sum_{i} \phi\left(\| \cdot-x_{i}||\right) f_{i}$. Com essa estratégia, extrapolamos os vetores $t_{i}$ 's em todo o domínio $X$, obtendo o campo:

$$
F_{\text {tan }}=\sum_{j}^{N_{b}} \phi\left(\left\|\cdot-y_{j}\right\|\right) t_{j}
$$

Finalmente, com relação à soma, queremos maior influência do campo $F_{\text {tan }}$ e menor influência do campo $F_{c t r}$ próximos ao contorno. Assim, podemos construir as funções pesos $w_{c t r}$ e $w_{\text {tan }}$, a partir da distância do contorno. Como o contorno $X_{b}$ considerado é discreto, podemos calcular de forma efetiva a distância por $d_{b}(x)=\min _{y \in X_{b}}(\|x-y\|)$ para todo $x \in X$ e então, normalizamos esses mapas para obter:

$$
\begin{aligned}
& \text { - } w_{c t r}(x)=d_{b}(x) / \max _{x \in X} d_{b}(x) \mathrm{e} \\
& \text { - } w_{t a n}(x)=1-w_{c t r}(x)
\end{aligned}
$$

A Figura 19 ilustra as funções pesos acima. Assim, com as equações 5.6 e 5.7, obtemos o campo resultante:

$$
F=w_{c t r}(\cdot) \sum_{j=1}^{N} \Phi_{d i v}\left(\left\|\cdot-x_{j}\right\|\right) c_{j}+w_{t a n}(\cdot) \sum_{j=1}^{N_{b}} \phi\left(\left\|\cdot-y_{j}\right\|\right) t_{j}
$$

A Figura 20 ilustra um exemplo da aplicação desse método com o mesmo domínio e discretização da seção anterior e mesma RBF $\phi_{3,3}$. Na Figura 20(a) temos a discretização do domínio com os pontos da fronteira identificados (vermelhos), as origens dos vetores de controle (circulados) e os pontos para avaliar o campo (pretos). Na Figura 20(b) iniciamos o método calculando o campo $F_{c t r}$ com os vetores de controle no interior (vermelhos). Lembrando que nessa etapa, não consideramos qualquer informação do domínio, apenas interpolamos os $N=7$ vetores de controle, resolvendo um sistema linear de dimensão $14 \times 14$ (ao invés de $400 \times 400$ ), e avaliamos em todos os pontos de $X$. Na Figura 20(c), calculamos do campo $F_{\text {tan }}$, sem resolver 
Figura 19 - Funções peso construídas a partir da distância da fronteira.

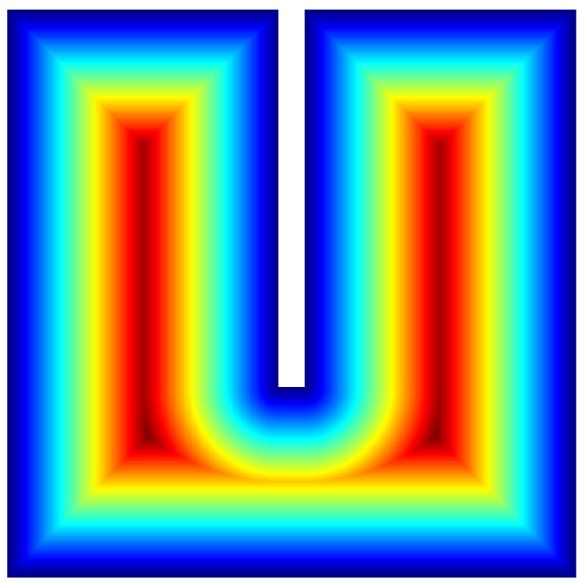

(a) Função $w_{c t r}$
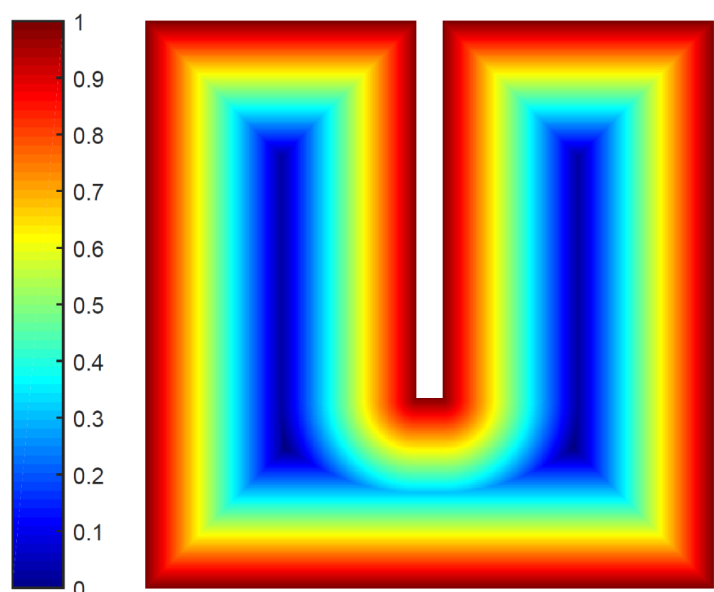

(b) Função $w_{\text {tan }}$

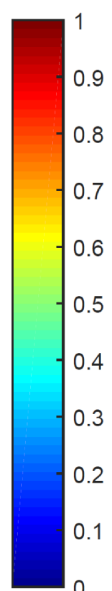

0.8

0.7

5 . 3 (1) .

Fonte: Elaborada pelo autor.

qualquer sistema, apenas avaliando a Equação 5.7 e finalmente, temos o campo resultante da soma representado na Figura 20(d).

\subsection{Restringindo o Domínio das RBFs}

No capítulo anterior, apresentamos o problema da vizinhança euclidiana em domínios côncavos e sua influência nas RBFs. Nesta seção, começaremos a explorar o uso de novas métricas aplicadas no método descrito na seção anterior. Para o cálculo do campo $F_{t a n}$, utilizamos a mesma abordadem de Levi e Levin (2014), apenas substituindo nas RBFs a distância euclidiana por alguma distância $d$ como a do método FMM ou a distância interior, ou seja, acoplamos diretamente a distância $d$ tomando $\phi_{j}:=\phi\left(\varepsilon d\left(\cdot, x_{j}\right)\right)$, e então obtendo:

$$
F_{\text {tan }}=\sum_{j}^{N_{b}} \phi_{j} t_{j} .
$$

A Figura 21 ilustra como esta mudança leva à melhoras significativas na aproximação. Nesta figura, notemos que com a distância euclidiana, utilizamos pontos os quais não deveriam ser considerados, pontos vermelhos na Figura 21(a), fazendo com que a Equação 5.9 produza uma aproximação pouco condizente com os vetores tangentes $t_{i}^{\prime} s$, como podemos ver na Figura 21(b), com o campo não respeitando o contorno. Já com a métrica intrínseca, consideramos pontos próximos pela geometria do domínio, pontos vermelhos na Figura 21(c) e portanto, a Equação 5.9 produz uma melhor aproximação, Figura 21(d).

É importante salientar que, o acoplamento da distância $d$ no campo $F_{c t r}$ não é tão simples de ser computado. Neste caso, necessitamos recalcular o núcleo $\Phi_{\text {div }}$ pela dependência das derivadas da distância. Nessa seção, propomos uma alternativa para utilizar as métricas 
Figura 20 - Exemplo do método proposto

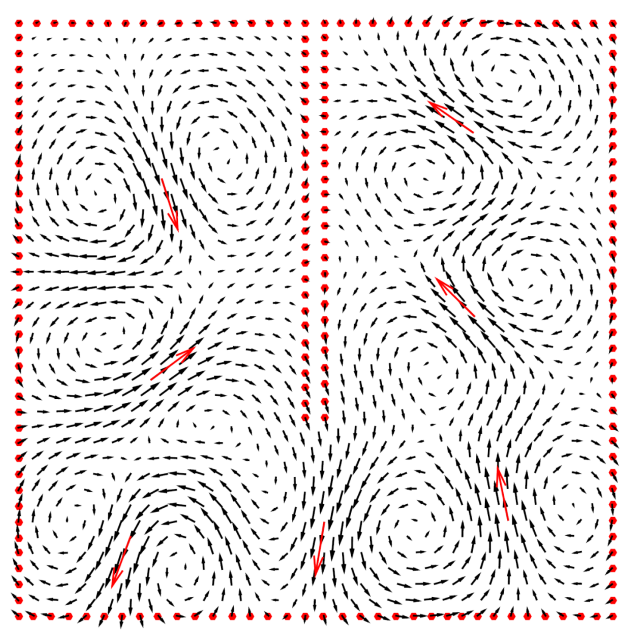

(a) Campo $F_{c t r}$

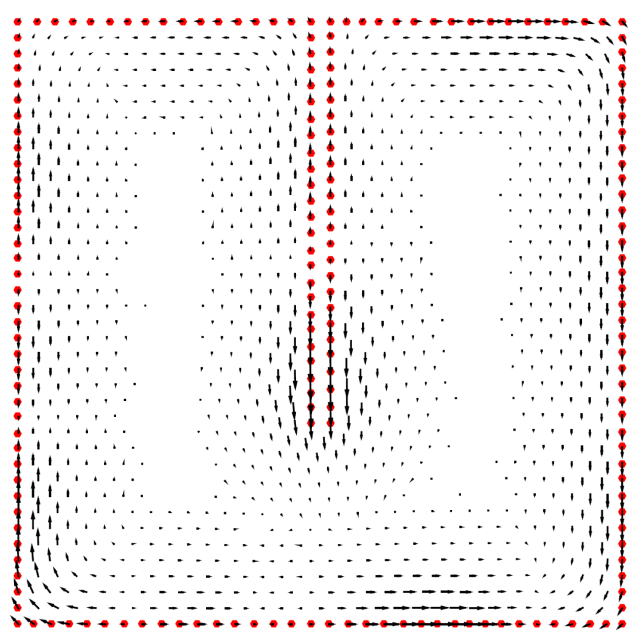

(b) Campo $F_{\text {tan }}$

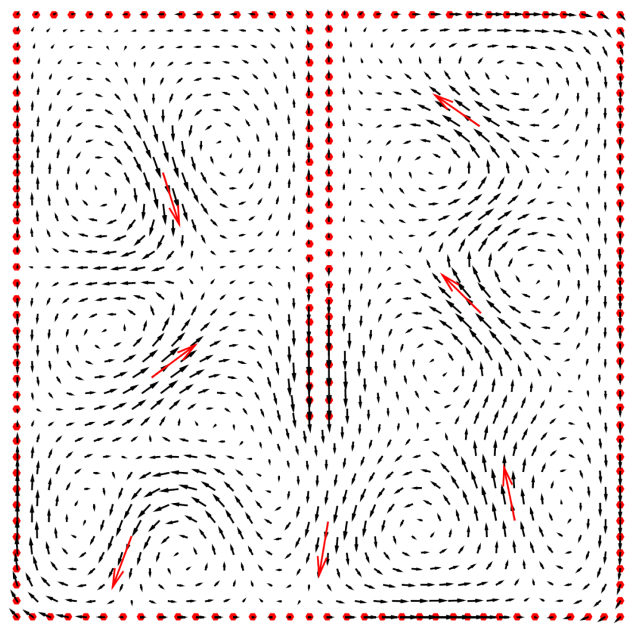

(c) Campo resultante

Fonte: Elaborada pelo autor.

intrínsecas em conjunto com a distância euclidiana sem a necessidade de recalcular o núcleo $\Phi_{d i v}$. A ideia consiste em restringir o suporte de cada função de base $\phi_{j}$ através de um mapa de distância interior $U_{j}: X \rightarrow \mathbb{R}$, tal que $U_{j}(x)=d\left(x, x_{j}\right)$. Optamos pelo mapa de distância interior $U$ dado pelo método FMM devido ao melhor desempenho computacional comparado com a distância de interior.

Para isso, seja $\phi_{j}$ uma CSRBF de Wendland com centro $x_{j}$ e parâmetro de forma $\varepsilon$ assim, o suporte de cada função $\phi_{j}$ é dado por:

$$
\begin{aligned}
\operatorname{supp}\left(\phi_{j}\right) & =\left\{x \in \mathbb{R}^{2} ;\left\|x-x_{j}\right\|_{2} \leq \rho_{1}=1 / \varepsilon\right\} \\
& =B_{\mathbb{R}^{2}}\left[x_{j}, \rho_{1}\right]
\end{aligned}
$$

isto é, a bola fechada de centro $x_{j}$ e raio $\rho_{1}$. Por outro lado, também temos a bola fechada 
Figura 21 - Influência da vizinhança no método de quasi-interpolação

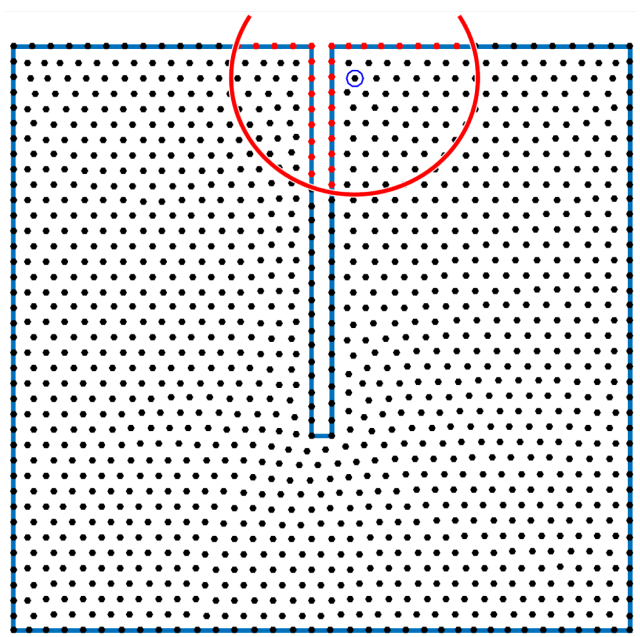

(a) Domínio discreto $X$

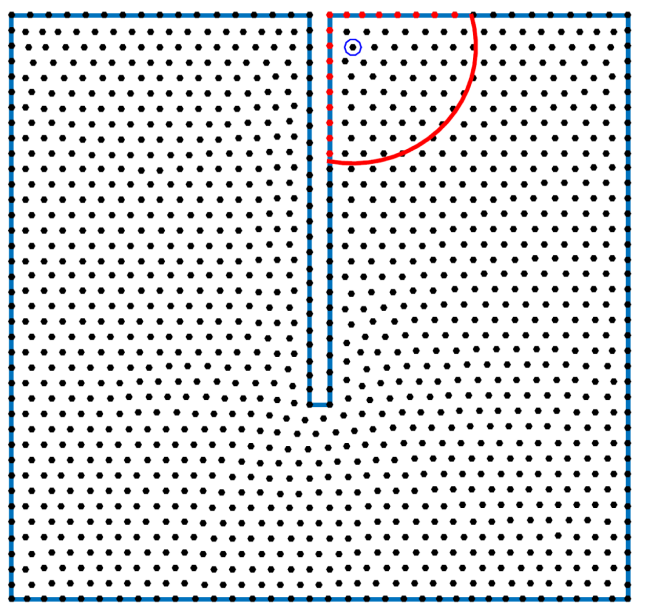

(c) Campo $F_{t a n}$

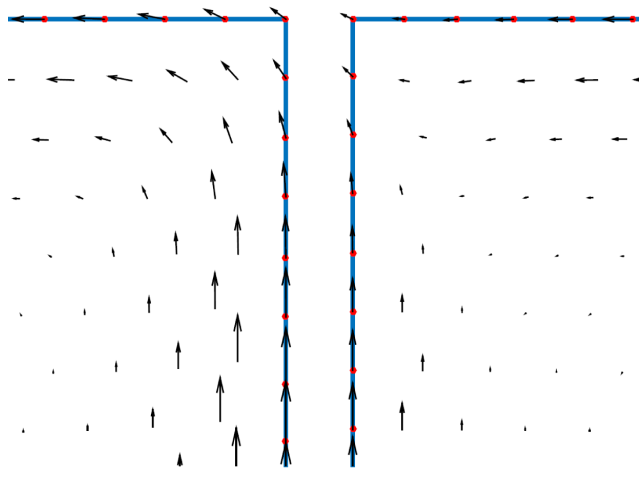

(b) Campo $F_{c t r}$

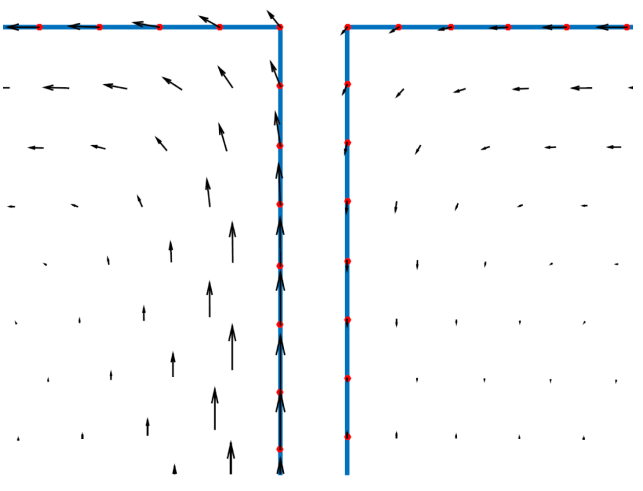

(d) Campo resultante

Fonte: Elaborada pelo autor.

associada ao mapa de distância $U$ :

$$
B_{U}\left[x_{j}, \rho_{2}\right]=\left\{x \in \mathbb{R}^{2} ; U_{x_{j}}(x) \leq \rho_{2}\right\}
$$

Assim, definimos o novo suporte de $\phi_{j}$ pela interseção desses conjuntos,

$$
\operatorname{supp}\left(\phi_{j}\right):=B_{\mathbb{R}^{2}}\left[x_{j}, \rho_{1}\right] \cap B_{U}\left[x_{j}, \rho_{2}\right] .
$$

A Figura 22 ilustra todo o processo de restrição descrito acima. Assim, podemos continuar utilizando o núcleo $\Phi_{d i v}$ como anteriormente, apenas considerando os novos suportes para as CSRBFS e desta forma, construir o campo $F_{c t r}$ com mais informações do domínio.

Este método pode ser útil em muitos casos, podendo ser aplicado em apenas alguns pontos específicos, além de ser de fácil implementação, entretanto a escolha dos raios $\rho_{1}$ e $\rho_{2}$ possuem restrições entre si. Note que, para $\rho_{2}<\rho_{1}$ destruímos o decaimento suave da RBF, 
Figura 22 - Restrição do suporte

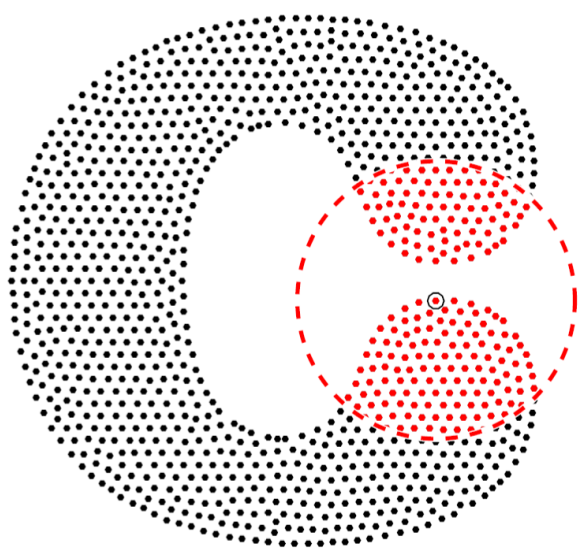

(a) $B_{\mathbb{R}^{2}}\left[x_{j}, \rho_{1}\right]$

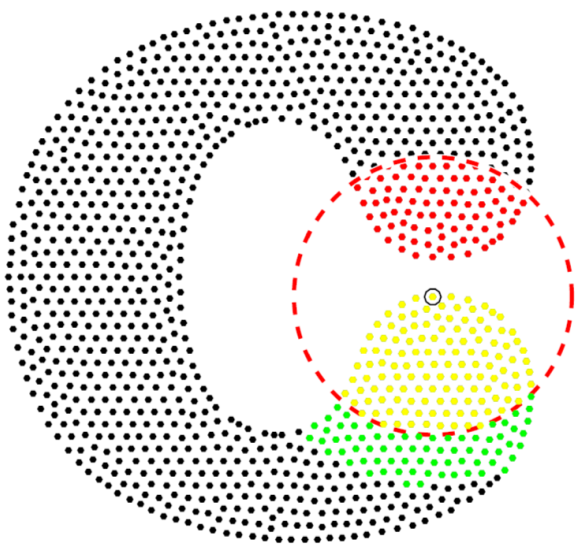

(c) $B_{\mathbb{R}^{2}}\left[x_{j}, \rho_{1}\right] \cap B_{U}\left[x_{j}, \rho_{2}\right]$

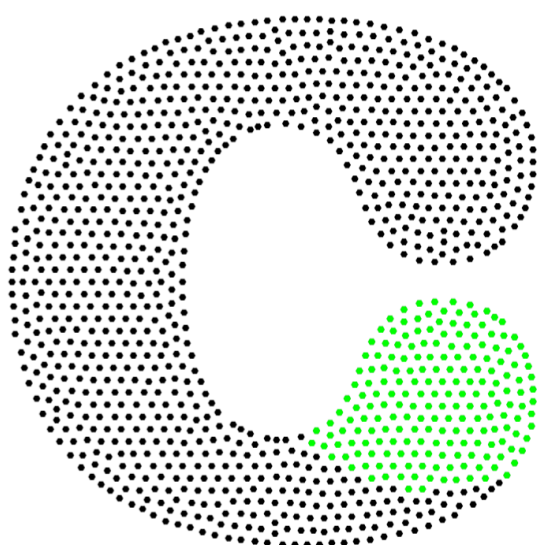

(b) $B_{U}\left[x_{j}, \rho_{2}\right]$

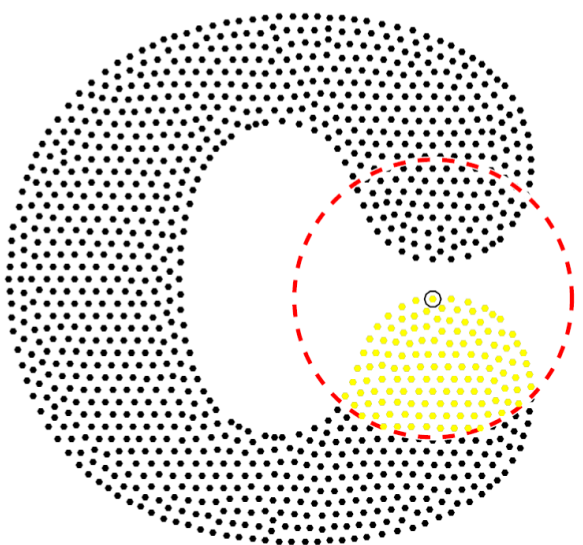

(d) $B_{\mathbb{R}^{2}}\left[x_{j}, \rho_{1}\right] \cap B_{U}\left[x_{j}, \rho_{2}\right]$

Fonte: Elaborada pelo autor.

podendo afetar na continuidade do interpolante. No experimento acima, consideramos $\rho_{2}=1.1 \rho_{1}$ mas esta heurística pode falhar para outros domínios.

\subsection{Acoplando Distância Interior em Núcleos MVRBF}

No método anterior, controlar $\rho_{1}$ e $\rho_{2}$ pode ser um grande problema em domínios mais complexos. Além disso, utilizamos uma métrica intrinseca apenas para restringir o domínio das funções de base, permanecendo as características da distância euclidiana, ou seja, não consideramos todo comportamento da nova distância avaliada. Para construir o núcleo MVRBF $\Phi_{d i v}$ utilizando novas métricas incorporadas, de fato, nas RBFs, consideramos a distância interior definida por Rustamov.

No Capítulo 3, apresentamos a distância interior definida por Rustamov, entre os pontos $p$ e $q$ de uma região poligonal fechada de vértices $v_{1}, \ldots, v_{n} \in \mathbb{R}^{2}$, dada pela distância euclidiana da imersão desses pontos em algum $\mathbb{R}^{m}$. Essa imersão é obtida através do mapa de coordenadas 
baricentricas $w: \mathbb{R}^{2} \rightarrow \mathbb{R}^{n}$ e pela matriz de Gram $A$, construida a partir das imersões dos vértices da região poligonal em $\mathbb{R}^{m}$. E assim, podemos escreve-la da forma:

$$
d(p, q)=\sqrt{[w(p)-w(q)]^{\top} A[w(p)-w(q)]} .
$$

Para o cálculo dos elementos do núcleo, sejam $w=w(p)-w(q)$ e $r=\left(w^{\top} A w\right)^{1 / 2}$. Como anteriormente, utilizamos o operador $\mathscr{D}$ para remover as singularidades fazendo $\mathscr{D}_{\phi}(r)=\frac{1}{r} \frac{d}{d r} \phi(r)$ e $\mathscr{D}_{\phi}^{2}(r)=\frac{1}{r} \frac{d}{d r} \mathscr{D}_{\phi}(r)$. Assim, obtemos as derivadas parciais de primeira ordem:

$$
\begin{aligned}
\frac{\partial}{\partial x} \phi(r) & =\frac{d}{d r} \phi(r) \frac{\partial}{\partial x} r \\
& =\frac{1}{r} \frac{d}{d r} \phi(r) w^{\top} A w_{x} \\
& =\mathscr{D}_{\phi}(r) w^{\top} A w_{x},
\end{aligned}
$$

sendo de forma análoga para a coordenada y, e as derivadas de segunda ordem:

$$
\begin{aligned}
\frac{\partial^{2}}{\partial x y} \phi(r) & =\left(\frac{\partial}{\partial y} \mathscr{D}_{\phi}(r)\right) w^{\top} A w_{x}+\mathscr{D}_{\phi}(r)\left(\frac{\partial}{\partial y}\left(w^{\top} A w_{x}\right)\right) \\
& =\frac{1}{r} \frac{d}{d r} \mathscr{D}_{\phi}(r)\left(w^{\top} A w_{y}\right)\left(w^{\top} A w_{x}\right)+\mathscr{D}_{\phi}(r)\left(w^{\top} A w_{x y}+w_{y}^{\top} A w_{x}\right) \\
& =\mathscr{D}_{\phi}^{2}(r)\left(w^{\top} A w_{x}\right)\left(w^{\top} A w_{y}\right)+\mathscr{D}_{\phi}(r)\left(w^{\top} A w_{x y}+w_{y}^{\top} A w_{x}\right),
\end{aligned}
$$

obtendo $\frac{\partial^{2}}{\partial x^{2}} \phi(r)$ e $\frac{\partial^{2}}{\partial y^{2}} \phi(r)$ apenas substituindo $x$ por $y$ na expressão acima. Assim, nos aproximamos de uma forma explícita dos elementos do núcleo, porém, em função das derivadas das coordenadas baricêntricas $w$. Essa dependência é um grande problema no momento, pois não conhecemos, de forma geral, essas derivadas e portanto, não conseguimos obter analiticamente o núcleo matricial. Entretanto, para estudos futuros, podemos partir desse ponto, buscando por expressões analíticas das diversas coordenadas baricentricas existentes na literatura.

Com a dificuldade de obter a forma analítica para o núcleo, partimos para aproximações numéricas das derivadas. A etapa fundamental no cálculo do núcleo é a remoção das singularidades das derivadas quando $r=0$, utilizando operador $\mathscr{D}$ e para isso, não precisamos nos preocupar com as coordenadas baricentricas, basta considerarmos a função $g=\left(w^{\top} A w\right)$ para conseguir a mesma simplificação ou seja, obtemos as derivadas parciais de segunda ordem em função das derivadas de $g$ :

$$
\begin{aligned}
\frac{\partial^{2}}{\partial x y} \phi(r) & =\frac{\partial}{\partial y}\left[\frac{d}{d r} \phi(r) \frac{\partial}{\partial x} r\right] \\
& =\frac{\partial}{\partial y}\left[\frac{1}{2} g_{x} \mathscr{D}_{\phi}(r)\right] \\
& =\frac{1}{2}\left[\frac{1}{2} g_{x} g_{y} \frac{1}{r} \frac{d}{d r} \mathscr{D}_{\phi}(r)+g_{x y} \mathscr{D}_{\phi}(r)\right] \\
& =\frac{1}{2}\left[\frac{1}{2} g_{x} g_{y} \mathscr{D}_{\phi}^{2}(r)+g_{x y} \mathscr{D}_{\phi}(r)\right],
\end{aligned}
$$


e de forma análoga obtemos os demais elementos do núcleo:

$$
\Phi_{d i v}(r)=\frac{1}{2}\left[\begin{array}{cc}
-\frac{1}{2} g_{y}^{2} \mathscr{D}_{\phi}^{2}(r)-g_{y y} \mathscr{D}_{\phi}(r) & \frac{1}{2} g_{x} g_{y} \mathscr{D}_{\phi}^{2}(r)+g_{x y} \mathscr{D}_{\phi}(r) \\
\frac{1}{2} g_{x} g_{y} \mathscr{D}_{\phi}^{2}(r)+g_{x y} \mathscr{D}_{\phi}(r) & -\frac{1}{2} g_{x}^{2} \mathscr{D}_{\phi}^{2}(r)-g_{x x} \mathscr{D}_{\phi}(r)
\end{array}\right] .
$$

Desta forma, precisamos aproximar $g_{x}, g_{y}, g_{x x}, g_{y y}$ e $g_{x y}$. Nesta etapa, avaliamos a função $g$ em todos os pontos de $X$ e então aproximamos esta função por polinômios cúbicos usando o método dos mínimos quadrados locais. Assim, obtemos facilmente aproximações para as derivadas com boa precisão. Com as aproximações, podemos dar suporte ao método proposto e assim, construir o campo $F_{\text {tan }}$, acoplando diretamente a distância $r=\left(w^{\top} A w\right)^{1 / 2}$ na RBF, e o campo $F_{c t r}$ utilizando o núcleo da Equação 5.13 juntamente com aproximações das derivadas.

Com o domínio utilizando anteriormente, a nova distância apresentou um mal comportamento indesejado, devido à proximidade das regiões não convexas, ilustrado na Figura 23(b). Portanto consideramos, nos teste, o domínio menos severo da Figura 23(a).

Figura 23 - Comportamento da distância ao aproximar regiões não convexas
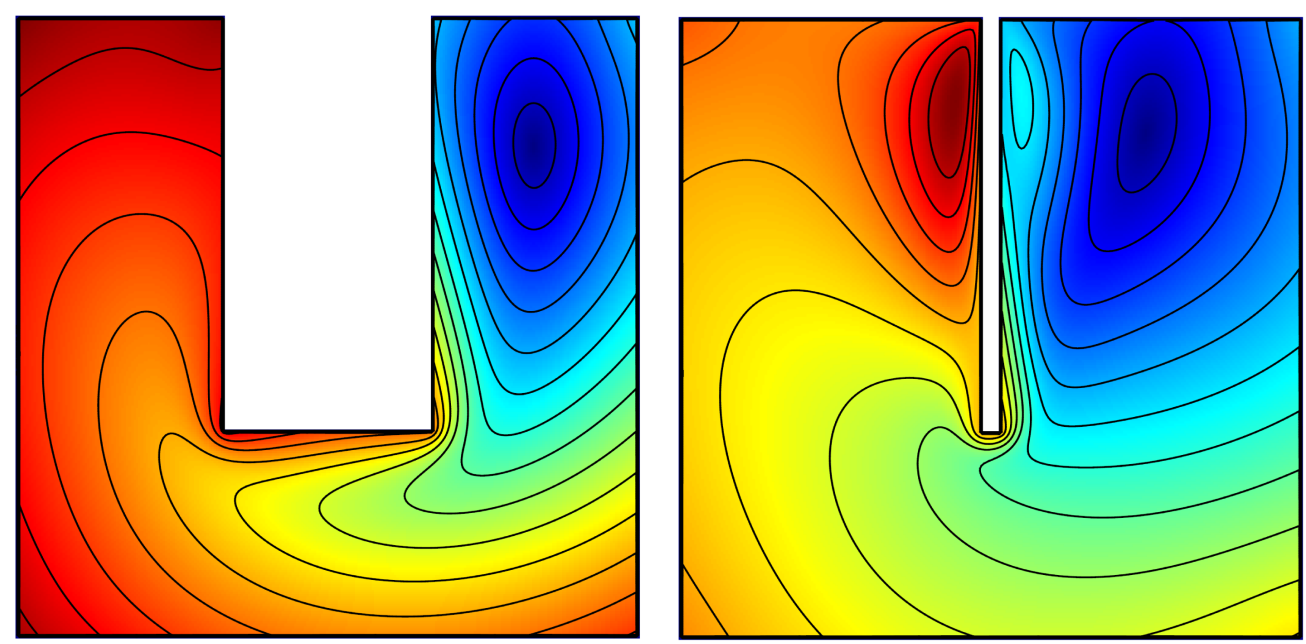

Fonte: Elaborada pelo autor.

Inicialmente, na Figura 24, construímos a interpolação de apenas um vetor de controle, utilizando o núcleo $\Phi_{\text {div }}$, a fim de verificar a influência da distância no interpolante, possibilitando a criação de novos padrões de vórtices. Entretanto, pelas linhas curvas de nível do mapa de distância, pode ser um problema controlar as regiões de influência das RBFs.

Na Figura 25, utilizamos o mesmo padrão dos métodos anteriores na distribuição dos vetores de controle e podemos notar regiões onde o interpolante não fica definido devido ao alto valores nas distâncias. Além disso, existem regiões críticas no domínio gerando vetores que desestabilizam todo o campo, possuindo magnitudes muito elevadas. Tal problema pode ser justificado pela magnitudes dos campos de derivadas $\left[g_{x} g_{y}\right]$ e $\left[g_{x x} g_{y y}\right]$ pois, refletem diretamente no interpolante. Na Figura 26 ilustramos a média dessas magnitudes. Este problema, permanece em outros domínios, como uma característica da própria distância, provavelmente herdada das coordenadas baricentras. 
Figura 24 - Influência da distância no núcleo
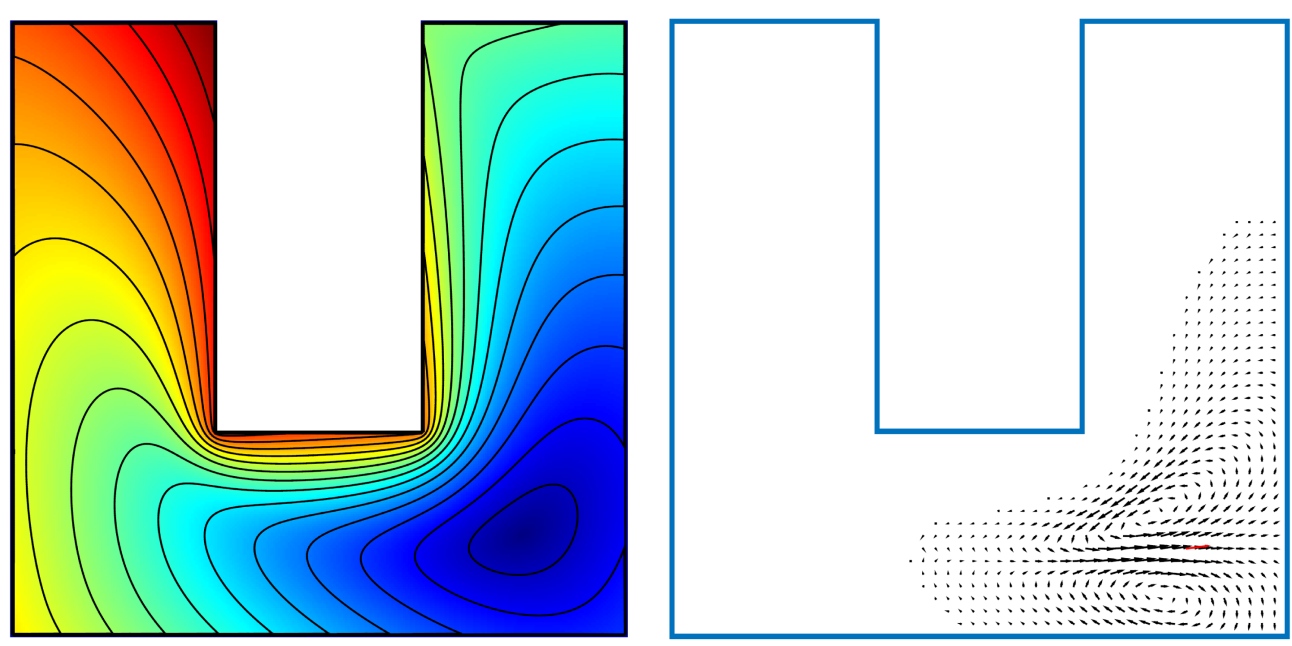

Fonte: Elaborada pelo autor.

Figura 25 - Tentativa de contruir o campo $F_{c t r}$ com a distância de Rustamov acoplada

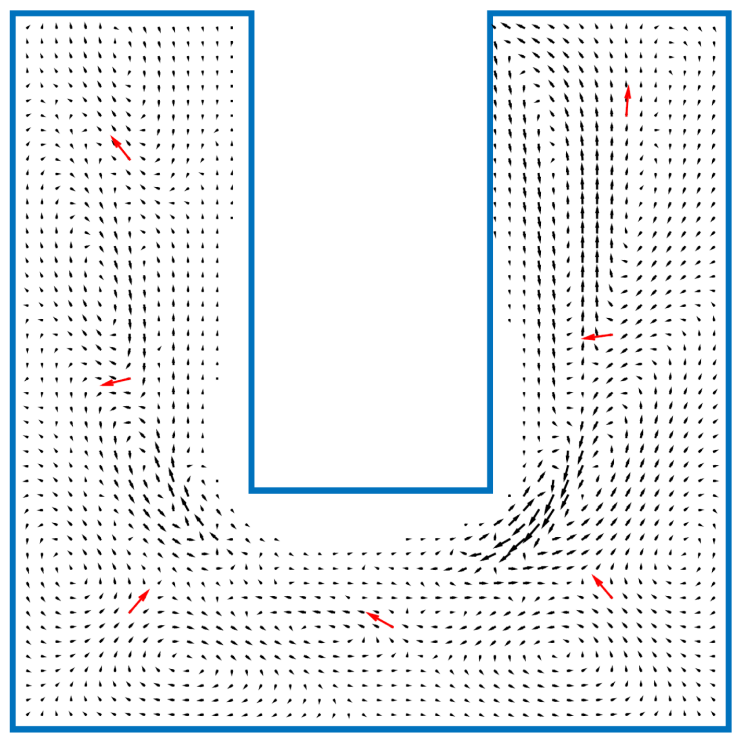

Fonte: Elaborada pelo autor.

\subsection{Núcleo MVRBF com FMM}

Nesta seção, continuamos com a mesma abordagem do método proposto porém desta vez, utilizando a distância provinda do método FMM. A distância definida por Rustamov tem a vantagem de ser um método sem malha assim como as RBFs. Por outro lado, o FMM necessita de uma malha triangular para ser calculado. Como apresentado anteriormente, o método FMM computa um mapa de distâncias $U_{j}: X \rightarrow \mathbb{R}$ através da solução de uma equação Eikonal na malha triangular. Cada mapa $U_{j}$ contém a distância interior de todo ponto $x \in X$ ao ponto de origem $x_{j}$. Aqui, também não temos as formas analíticas para as derivadas da distância assim, utilizamos o mesmo processo com mínimos quadrados para aproximar as derivadas tomando 
Figura 26 - Média das magnitudes das derivadas
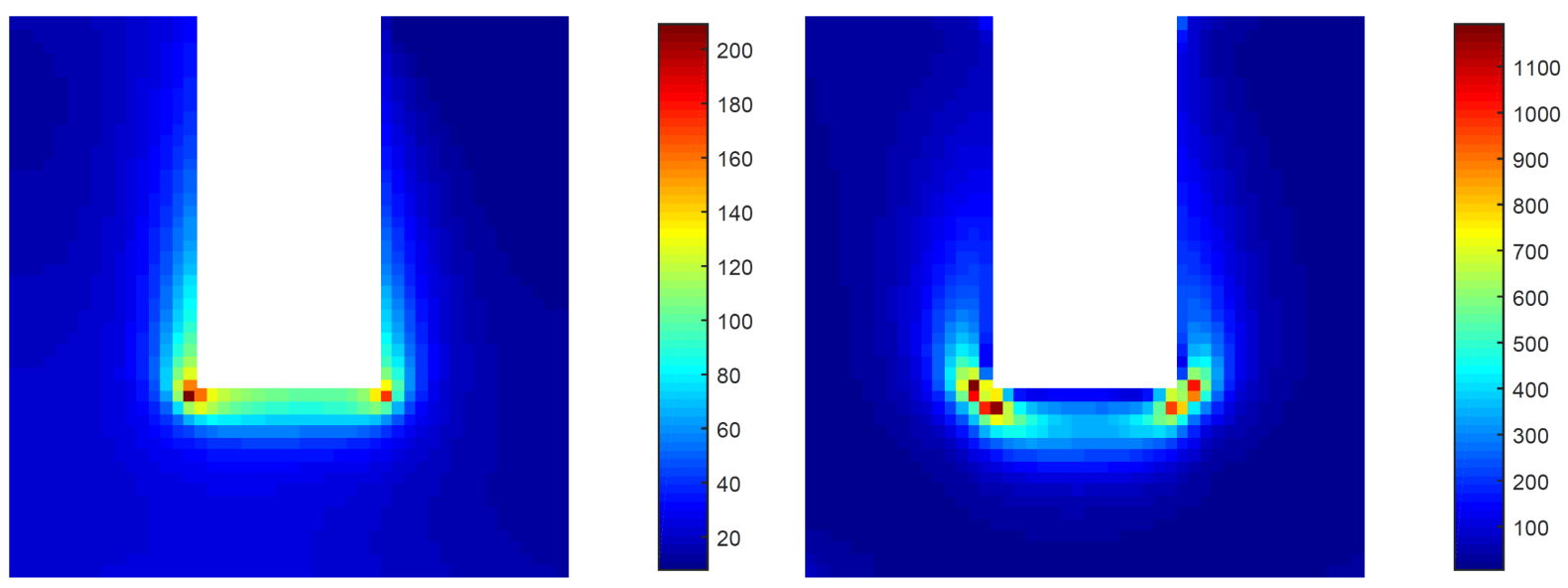

Fonte: Elaborada pelo autor.

como dados $g=U^{2}$. Desta forma, evitamos as singularidades da distância e utilizamos o mesmo núcleo da Equação 5.13.

Na Figura 27(a) temos a malha triangular do domínio com os pontos de contorno (vermelhos) e as origens dos vetores de controle. Já na Figura 27(b), ilustramos o mapa de distâncias $U_{j}$ com a origem $x_{j}$ (ponto vermelho) e alguns vetores gradientes desse mapa, obtidos pela aproximação numérica.

Figura 27 - Malha triangular do domínio
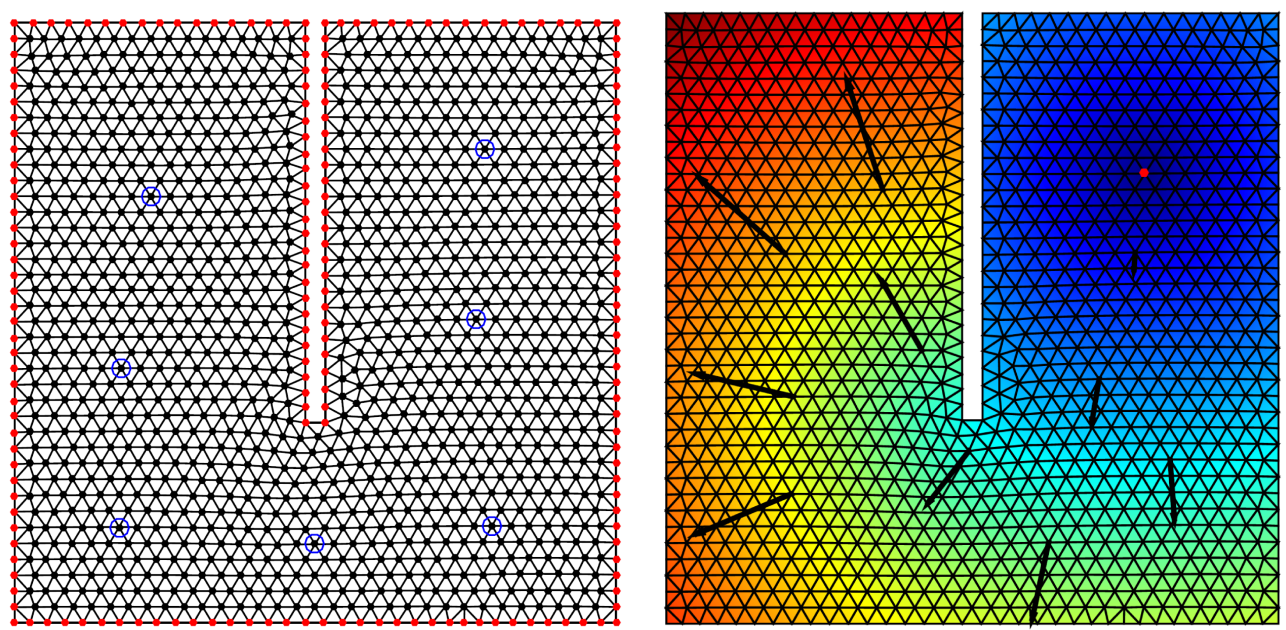

Fonte: Elaborada pelo autor.

A construção do campo $F_{c t r}$ segue similar ao método anterior, com núcleo da Equação 5.13, obtemos o interpolante dos vetores de controle $v_{j}$ 's em todo o domínio X, ver Figura 28(a). Na Figura 28(b) temos o campo $F_{t a n}$, obtido como anteriormente, apenas acoplamos os mapas $U_{j}$ nas RBFs do método de quasi-interpolação. E finalmente, na Figura 28(c), temos o campo resultante. Na Figura 28(d) utilizamos a técnica de visualização de campos vetoriais 
Line Integral Convolution(LIC) Cabral e Leedom (1993), implementada do software Paraview. A Figura 31 ilustra todas as etapas do método em um domínio mais simples, utilizando uma discretização com 1382 pontos e 7 vetores de controle. Os demais parâmetros foram os mesmos.

Figura 28 - Exemplo do método proposto com a distância do FMM acoplada.

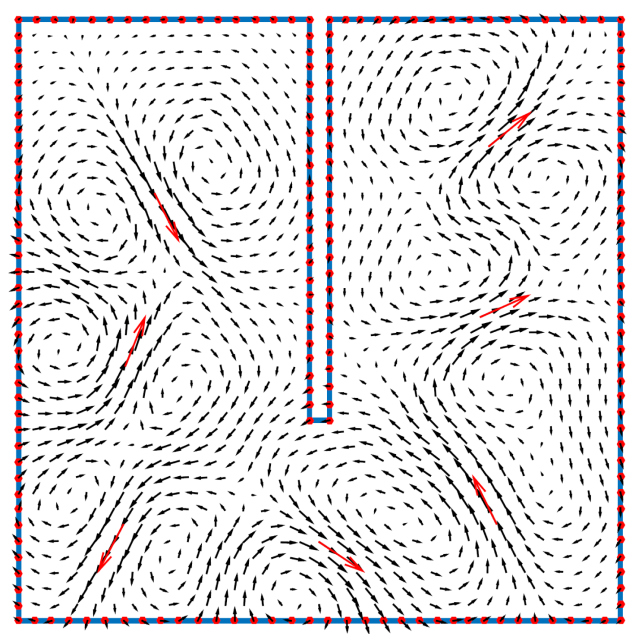

(a) Campo $F_{c t r}$

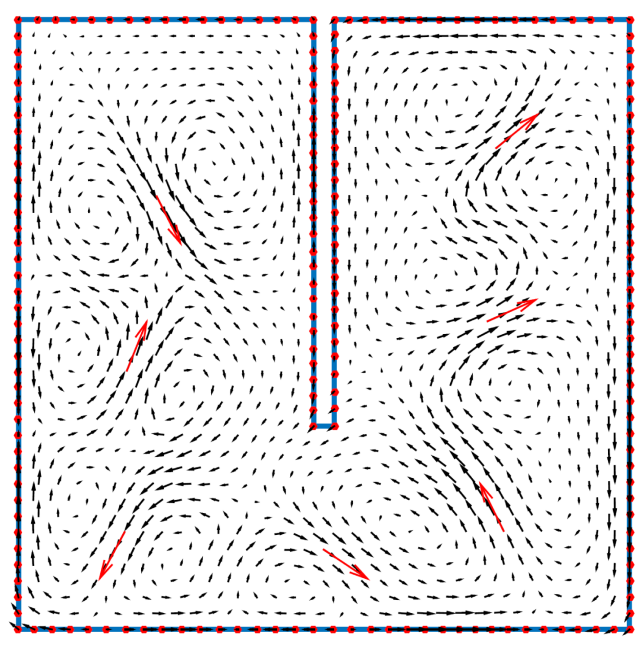

(c) Campo resultante

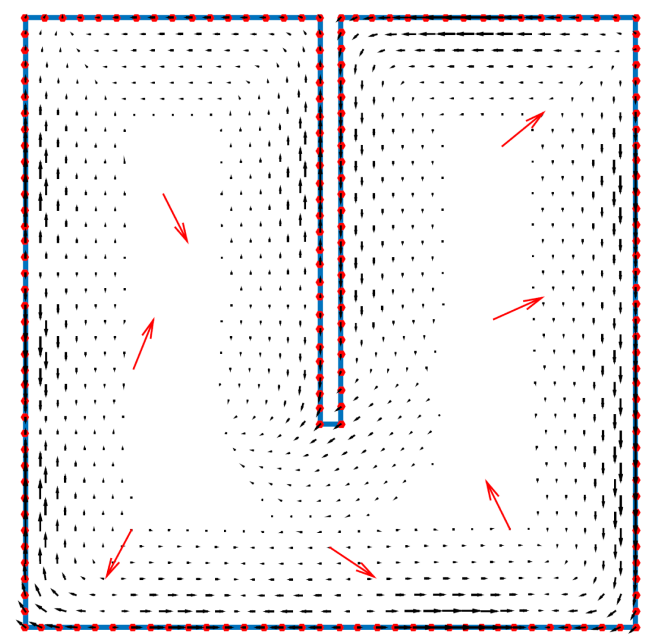

(b) Campo $F_{t a n}$

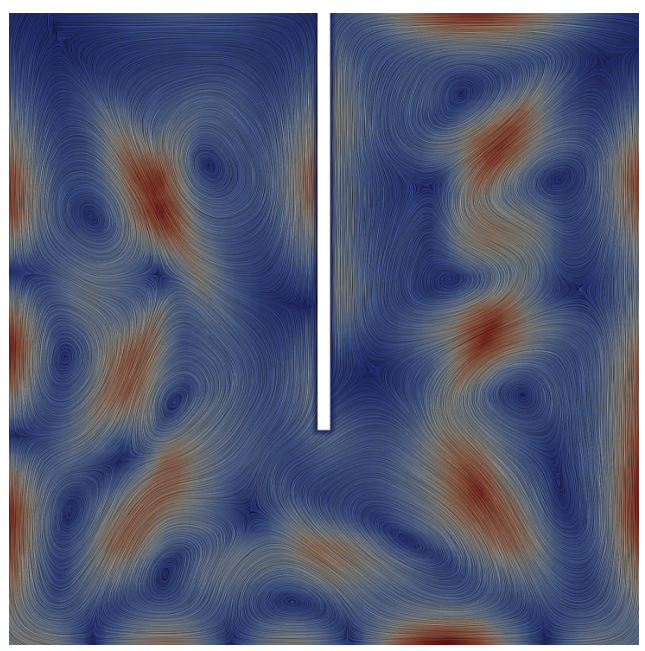

(d) Visualização com o LIC

Fonte: Elaborada pelo autor.

\subsection{Considerações}

A abordagem proposta neste capítulo, considera o campo vetorial final como a soma de dois campos $F_{c t r}$ e $F_{t a n}$, ponderados por funções peso $w_{c t r}$ e $w_{t a n}$ pela Equação 5.5, descrita na Seção 5.2. Estas funções peso ficaram bem determinadas através da distância da fronteira, enquanto que a construção dos campos $F_{c t r}$ e $F_{\text {tan }}$ sofreram variações nas demais seções com o objetivo de acoplar ao método distâncias intrínsecas do domínio. No cálculo do campo $F_{\text {tan }}$, optamos pelo uso da técnica de quasi-interpolação via RBF para aproximar os vetores tangentes 
Figura 29 - Exemplo do método proposto num domínio mais suave.

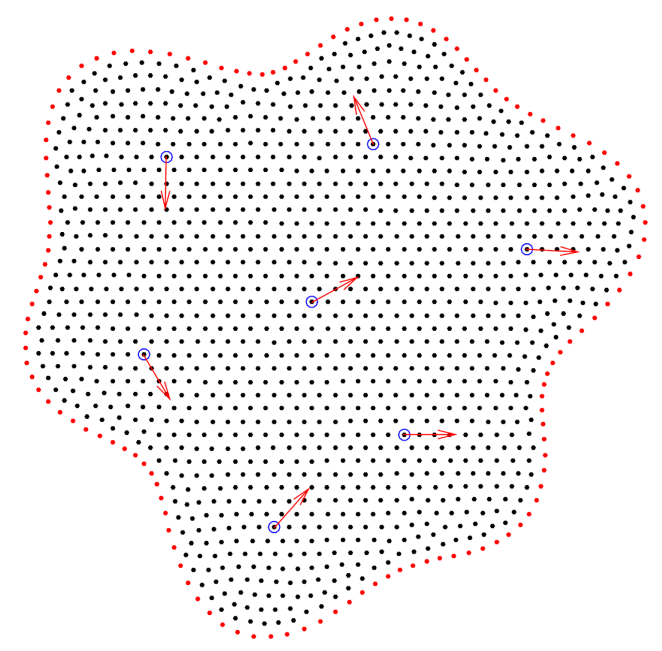

(a) Domínio discreto e vetores de controle

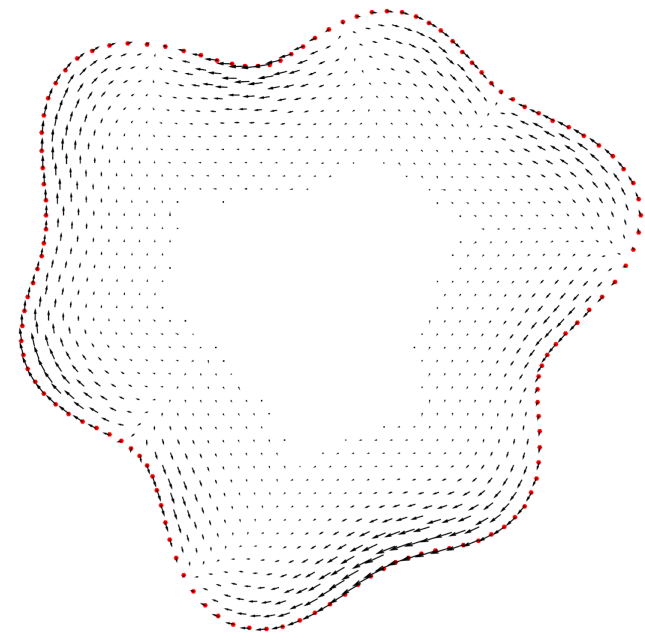

(c) Campo $F_{t a n}$

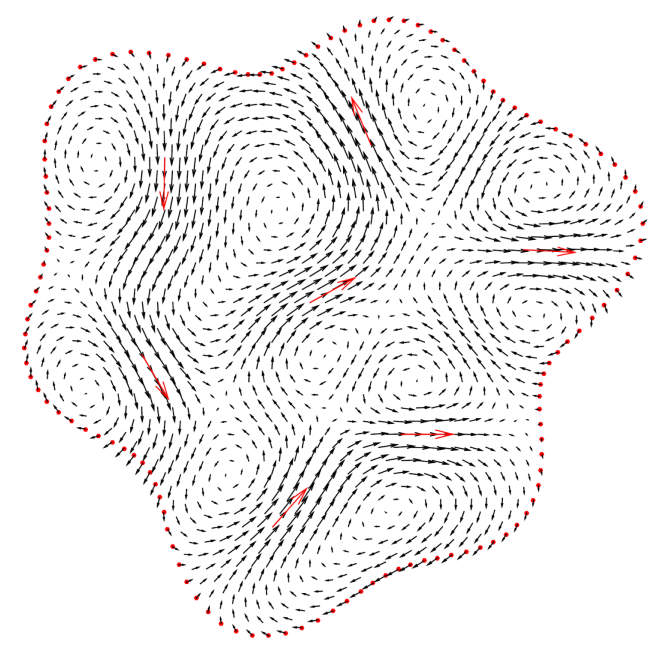

(b) Campo $F_{c t r}$

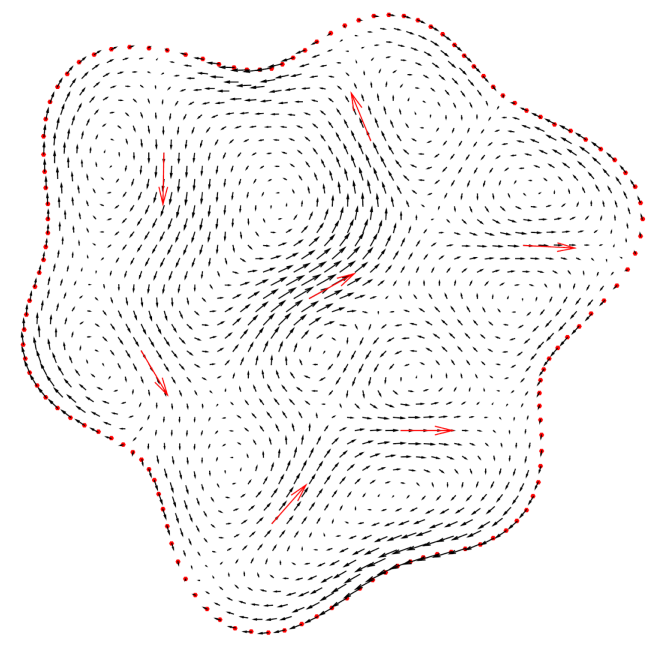

(d) Campo resultante $F$

Fonte: Elaborada pelo autor.

da fronteira, evitando a solução de um sistema linear com muitas equações e mal condicionado, porém foi necessário o uso da distância interior provida do método FMM para manter a fidelidade do contorno. Já o campo $F_{c t r}$, utiliza o núcleo matricial de divergente nulo o qual faz o uso, originalmente, da distância euclidiana. Na Seção 5.3, propomos uma forma de considerar uma métrica intrínseca porém mantendo o uso da distância euclidiana no núcleo. Esta abordagem se mostrou útil apenas em domínios específicos além de termos um novo parâmetro de ajuste. Já na Seção 5.4, iniciamos o acoplamento de novas métricas no núcleo matricial com a distância interior via coordenadas baricentricas (Rustamov) porém, sem êxito. Na seção seguinte, com a distância provida do método FMM, o acoplamento foi bem sucedido resultando em campos vetorias contínuos, com divergente nulo e com influência da métrica intrínseca. Com tudo é necessário aproximar as derivadas de segunda ordem desta nova métrica, afetando o desempenho.

Em resumo, temos duas opções para o cálculo de $F_{c t r}$ : com a distância euclidiana, 
possuindo expressões analíticas para as derivadas, mas não considerando a concavidade das regiões ou com a distância do método FMM, onde consideramos uma métrica intrínseca do domínio, porém com maior custo computacional para aproximar as derivadas da distância. Assim, o campo resultante do método proposto possui a seguinte forma:

$$
F=w_{c t r}(\cdot) \sum_{j=1}^{N} \Phi_{d i v}(r) c_{j}+w_{t a n}(\cdot) \sum_{j=1}^{N_{b}} \phi\left(d_{F M M}\left(\cdot, y_{j}\right)\right) t_{j}
$$

em que:

- Para $r=\left\|\cdot-x_{j}\right\|_{2}$, consideramos o núcleo:

$$
\Phi_{d i v}(r)=\left[\begin{array}{cc}
-y^{2} \mathscr{D}_{\phi}^{2}(r)-\mathscr{D}_{\phi}(r) & x y \mathscr{D}_{\phi}^{2}(r) \\
x y \mathscr{D}_{\phi}^{2}(r) & -x^{2} \mathscr{D}_{\phi}^{2}(r)-\mathscr{D}_{\phi}(r)
\end{array}\right]
$$

- Para $r=d_{F M M}\left(\cdot, x_{j}\right)$, consideramos o núcleo:

$$
\Phi_{\text {div }}(r)=\frac{1}{2}\left[\begin{array}{cc}
-\frac{1}{2} g_{y}^{2} \mathscr{D}_{\phi}^{2}(r)-g_{y y} \mathscr{D}_{\phi}(r) & \frac{1}{2} g_{x} g_{y} \mathscr{D}_{\phi}^{2}(r)+g_{x y} \mathscr{D}_{\phi}(r) \\
\frac{1}{2} g_{x} g_{y} \mathscr{D}_{\phi}^{2}(r)+g_{x y} \mathscr{D}_{\phi}(r) & -\frac{1}{2} g_{x}^{2} \mathscr{D}_{\phi}^{2}(r)-g_{x x} \mathscr{D}_{\phi}(r)
\end{array}\right] .
$$

Na próxima seção, seguem os resultados de alguns experimentos e os aspéctos computacionais, abordando as duas formas do método proposto descrito acima.

\subsection{Resultados}

Para a realização dos testes computacionais, os métodos foram implementados no software MATLAB R2015a e conduzidos num computador equipado com: um processador Intel Core 2 Quad de $2.66 \mathrm{GHz}, 4 \mathrm{~Gb}$ de memória RAM e o sistema operacional Windows 7. Os domínios foram gerados extraindo o contorno de imagens binárias, possibilitando a contrução de malhas triangulares com o pacote de geração de malhas distmesh ${ }^{2}$. Com isso, obtemos o domínio discreto $X$ e uma malha triangular para o método $\mathrm{FMM}^{3}$. Todos os domínios foram mapeados para $[-1,1]^{2}$ e em todos os casos foram utilizamos a seguinte CSRBF de Wendland e suas derivadas:

- $\phi_{3,3}(r)=(1-\varepsilon r)_{+}^{8}\left[32(\varepsilon r)^{3}+25(\varepsilon r)^{2}+8 \varepsilon r+1\right]$

- $\left.\mathscr{D}_{\phi}(r)=-22 \varepsilon^{2}(1-\varepsilon r)_{+}^{7}\left[16(\varepsilon r)^{2}+7 \varepsilon r+1\right)\right]$

- $\mathscr{D}_{\phi}^{2}(r)=528 \varepsilon^{4}\left[(1-\varepsilon r)_{+}^{6}(6 \varepsilon r+1)\right]$

2 http://persson.berkeley.edu/distmesh/

3 https://github.com/gpeyre/numerical-tours 
com os parâmetros de forma foram: $\varepsilon_{c t r}=2.0$ e $\varepsilon_{t a n}=6.0$ para o cálculo de $F_{c t r}$ e $F_{t a n}$ respectivamente.

Inicialmente, na Figura 30, ilustramos a influência da distância na construção de $F_{c t r}$. Neste exemplo, temos um domínio bem complexo, com regiões côncavas muito próximas. Podemos observar que utilizando a distância interior no campo $F_{c t r}$, temos melhor controle das regiões de atuação dos vetores de controle.

Figura 30 - Influência no campo final quando alteramos a distância na construção de $F_{c t r}$.

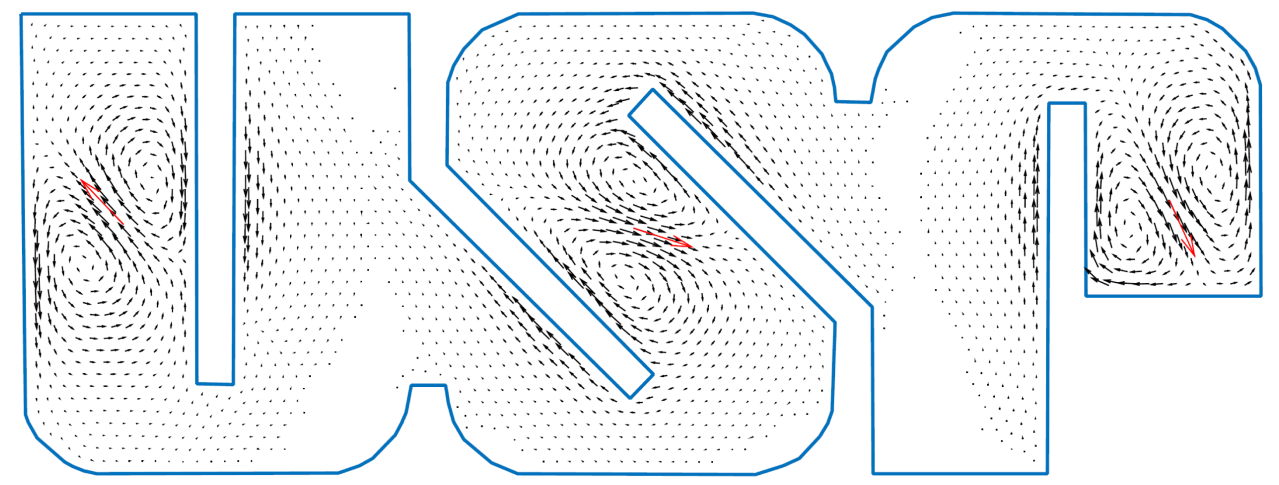

(a) $F_{c t r} \operatorname{com}\|\cdot\|_{2}$

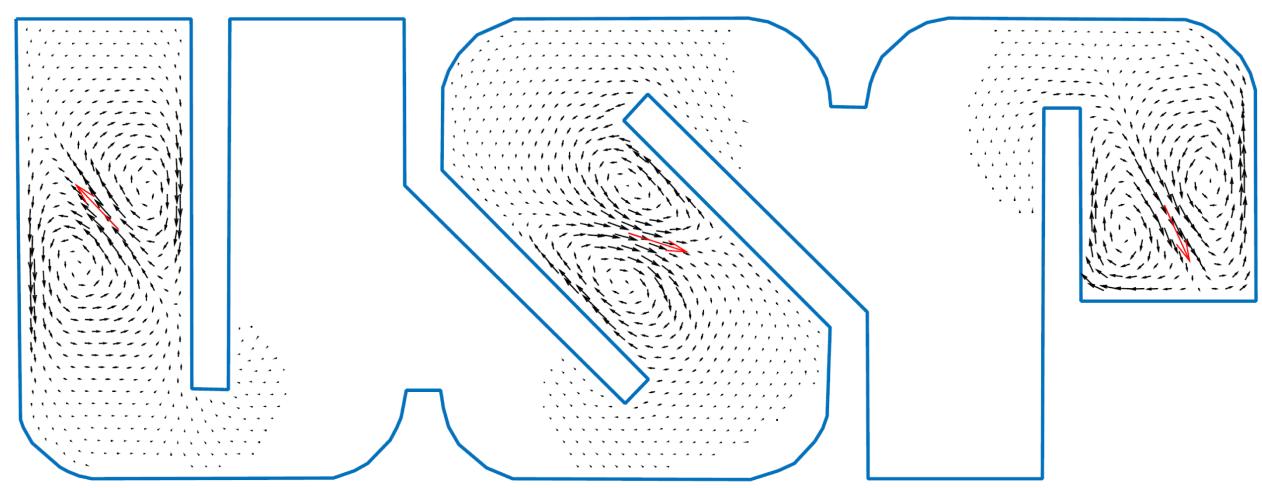

(b) $F_{c t r} \operatorname{com} d_{F M M}$

Fonte: Elaborada pelo autor.

Na Tabela 3, listamos os demais domínios testados e alguns resultados quantitativos obtidos com as duas métricas $\|\cdot\|_{2}$ e $d_{F M M}$ no cálculo do campo $F_{c t r}$. Nesta tabela, temos a média do valor absoluto do divergente em todos os pontos do domínio a fim de mostrar um certo controle sobre o divergente, pois a soma do campo tangente $F_{\text {tan }}$ afeta a propriedade de divergência nula de $F_{c t r}$ entretanto, são mantidas as características visuais. Também na tabela, segue o tempo computacional, em segundos, de ambos os métodos. Vale lembrar, que o cálculo de $F_{c t r}$ com a distância $d_{F M M}$ envolve aproximações numéricas para as derivadas da distância em todos os pontos elevando o custo computacional.

Durante os testes, verificamos que nem todo domínio não convexo necessita utilizar a distância interior $d_{F M M}$ no cálculo do campo $F_{c t r}$. Na Figura 31, temos um exemplo onde não 
Tabela 3 - Resultados obtidos com as métricas: $\|\cdot\|_{2}$ e $d_{F M M}$ no cálculo do campo $F_{c t r}$.

\begin{tabular}{|c|c|c|c|c|c|c|}
\cline { 4 - 7 } \multicolumn{2}{c|}{} & \multicolumn{2}{c|}{$F_{c t r}$ com $\|\cdot\|_{2}$} & \multicolumn{2}{c|}{$F_{c t r}$ com $d_{F M M}$} \\
\hline Domínios & $|X|$ & $\left|X_{c}\right|$ & Média $|\nabla \cdot|$ & Tempo (s) & Média $|\nabla \cdot|$ & Tempo (s) \\
\hline Fig. 31(a)(b) & 3839 & 11 & 0.8370 & 0.0792 & 0.8474 & 16.1387 \\
\hline Fig. 32(a)(b) & 3578 & 16 & 1.3713 & 0.0891 & 1.5059 & 21.1946 \\
\hline Fig. 33(a)(b) & 3076 & 18 & 1.2145 & 0.0883 & 2.1134 & 20.2770 \\
\hline Fig. 33(c)(d) & 2456 & 13 & 1.4243 & 0.0535 & 1.5563 & 11.6826 \\
\hline Fig. 33(e)(f) & 3353 & 21 & 1.6793 & 0.0897 & 1.8000 & 25.7516 \\
\hline
\end{tabular}

há diferenças visuais significativas em permanecer com a métrica euclidiana em $F_{c t r}$. Por outro lado, na Figura 32, esta a métrica intrínseca trouxe mudanças no comportamento do campo final em algumas regiões. A Figura 33 reune os demais exemplos com o mesmo comparativo.

Figura 31 - Exemplo de um domínio não convexo em que não há diferenças visuais significativas em utilizar a $d_{F M M}$.

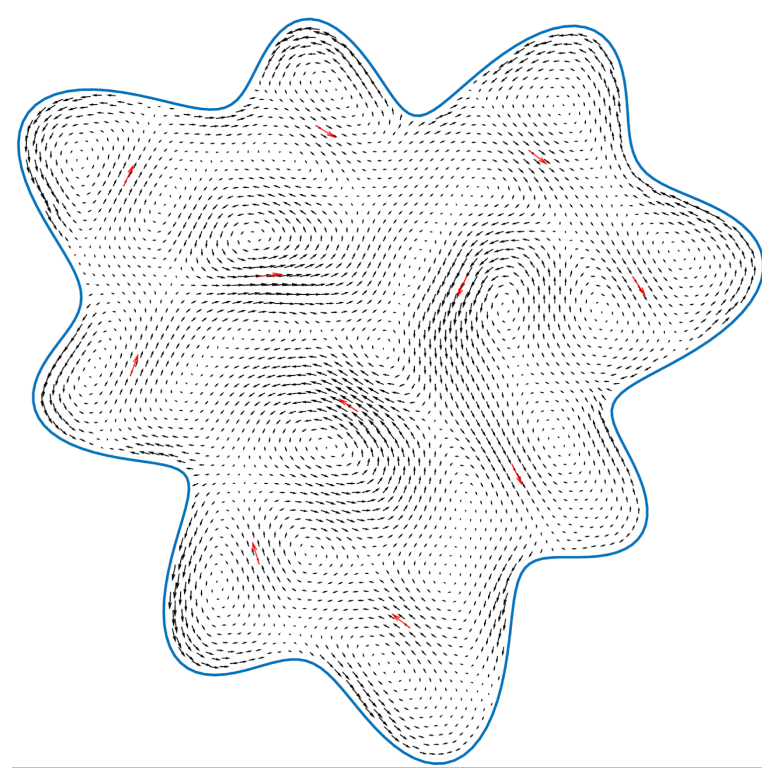

(a) $F_{c t r} \operatorname{com}\|\cdot\|_{2}$

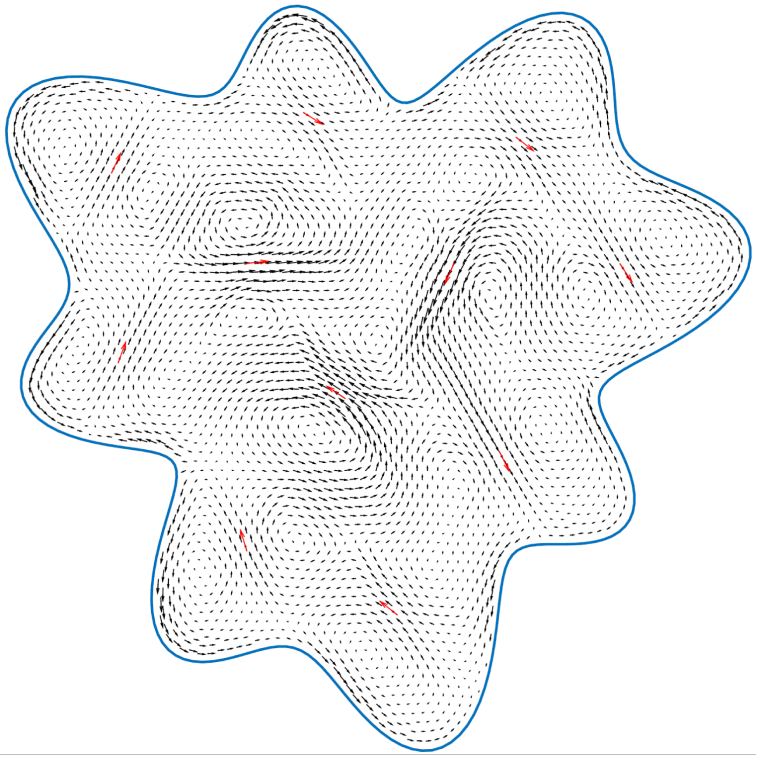

(b) $F_{c t r} \operatorname{com} d_{F M M}$

Fonte: Elaborada pelo autor. 
Figura 32 - Domínio com algumas melhorias visuais, porém com maior custo computacional.

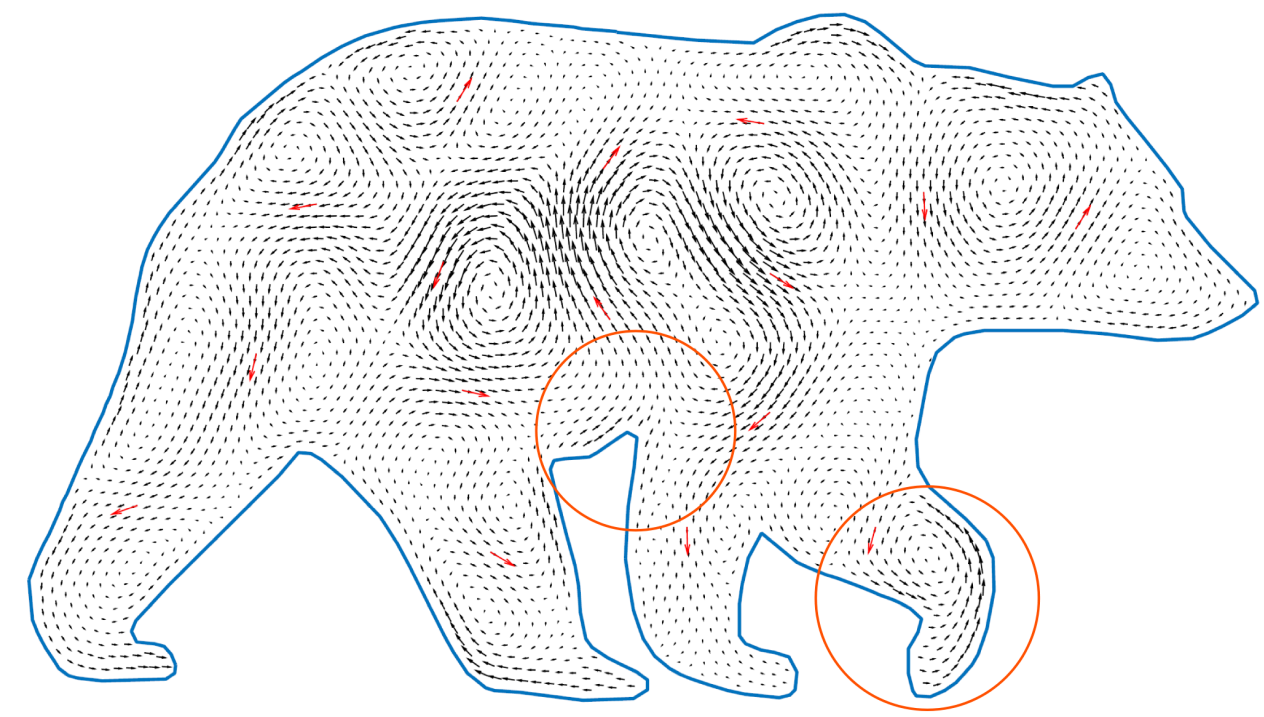

(a) $F_{c t r} \operatorname{com}\|\cdot\|_{2}$

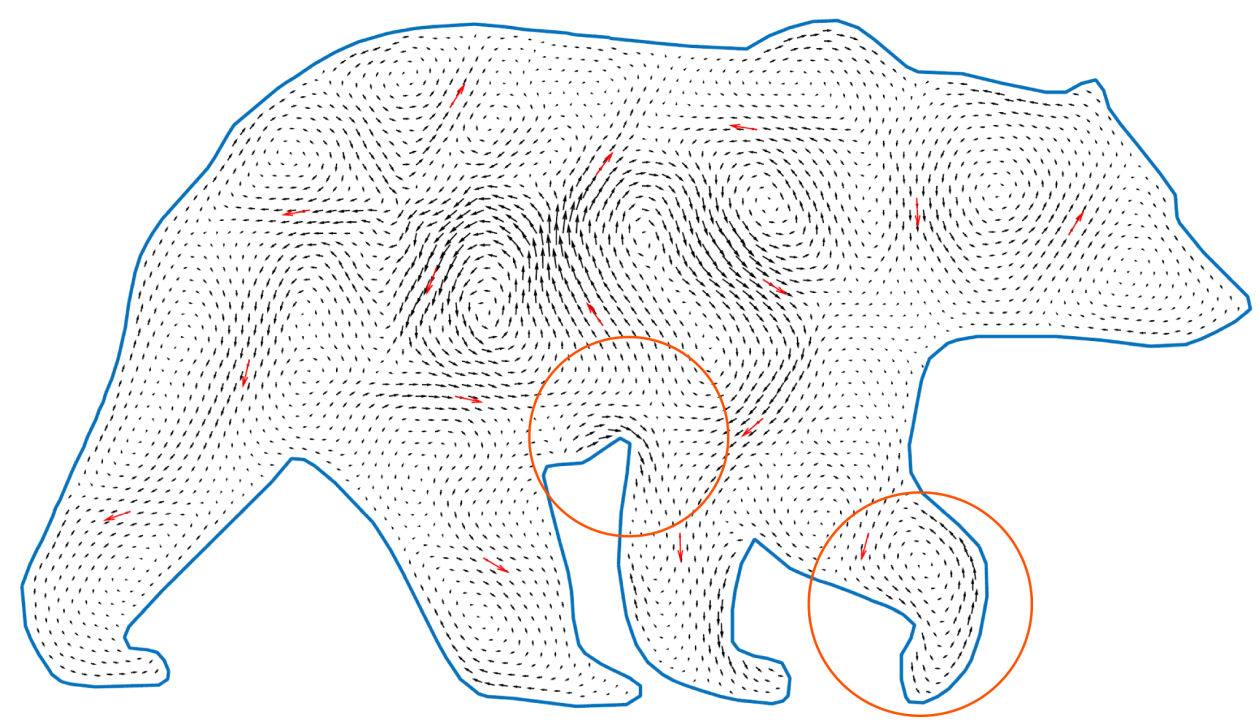

(b) $F_{c t r} \operatorname{com} d_{F M M}$

Fonte: Elaborada pelo autor. 
Figura 33 - Testes com as duas abordagens propostas. As figuras da esquerda, utilizam a distância euclidiana $\|\cdot\|_{2}$ na construção do campo $F_{c t r}$. Já as da direita utilizam a distância $d_{F M M}$.

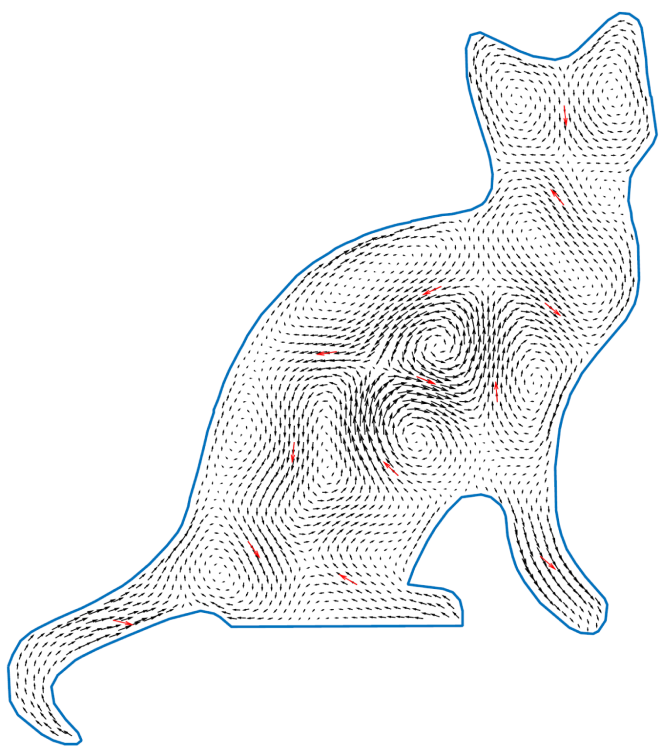

(a)

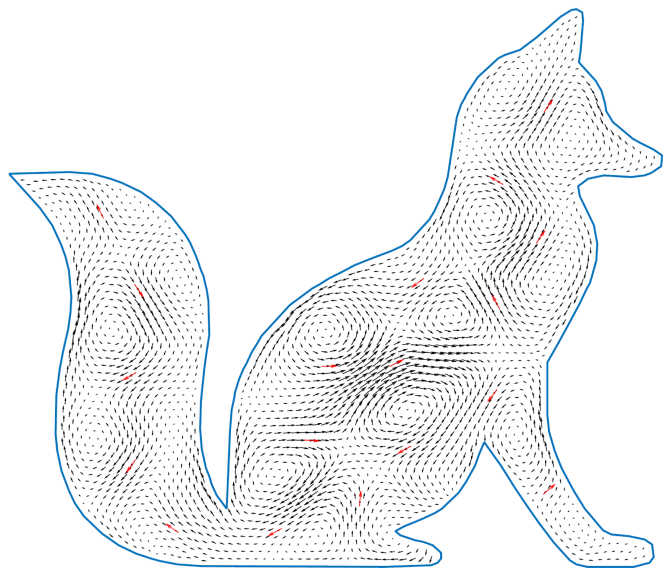

(c)

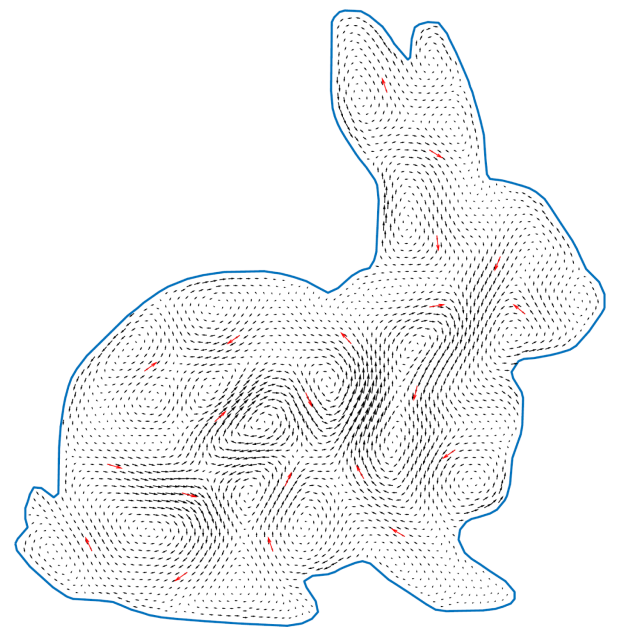

(e)

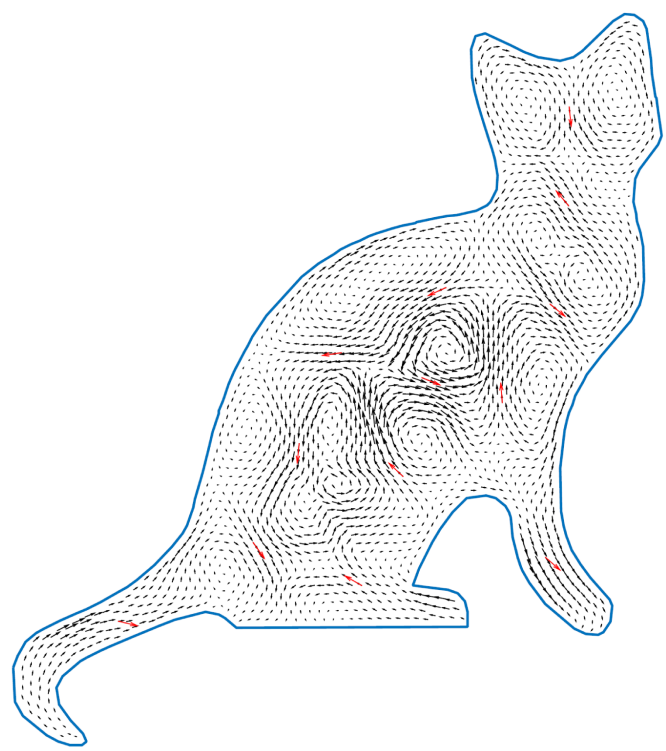

(b)

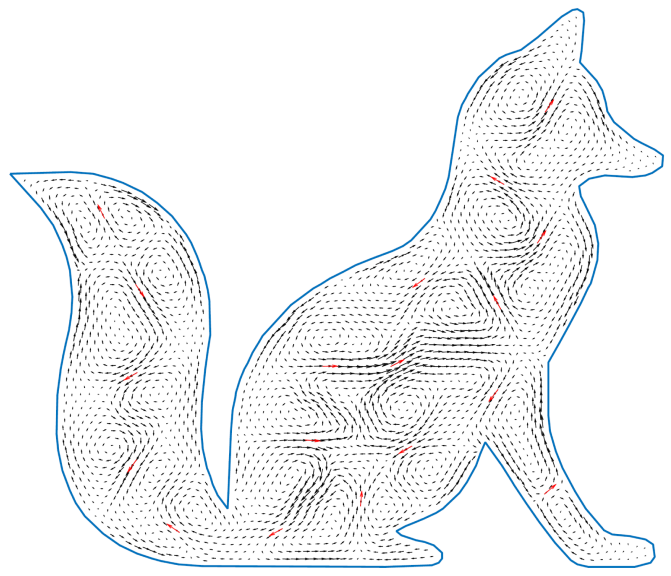

(d)

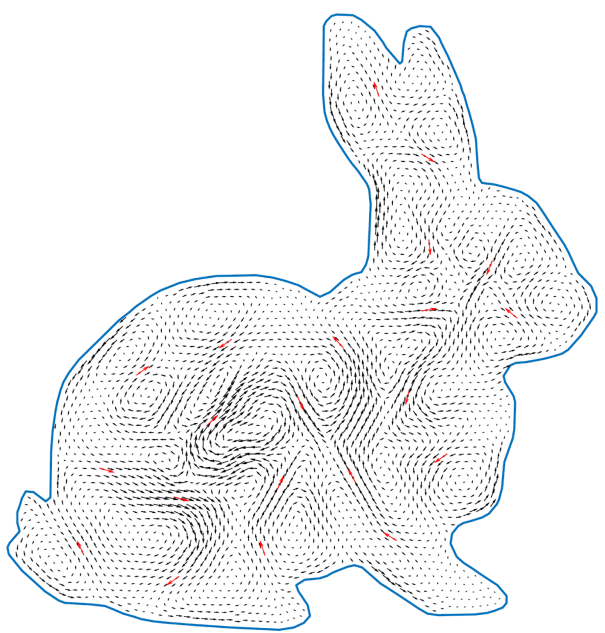

(f)

Fonte: Elaborada pelo autor. 



\section{CONCLUSÕES E PERSPECTIVAS}

Nessa dissertação, foi estudado o problema de design de campos vetoriais no interior de regiões do plano. Como o método proposto não segue a direção dos métodos construidos em malhas, sua eficiência e viabilidade foram as maiores preocupações. Seu desenvolvimento manteve o foco na possibilidade de design e na obtenção de caracteristicas visuais de fluídos incompressíveis, buscando, sempre que possível, abordagens livres de malhas. Estas propriedades direcionaram o início do estudo para problemas de interpolação de dados escalares com RBFs, chegando à interpolação de funções vetoriais utilizando o núcleo MVRBF introduzido por Narcowich e Ward (1994).

A primeira dificuldade enfrentada foi em manter o campo vetorial restrito ao interior do domínio. Para isso, testamos uma solução imediata, consistindo em apenas interpolar diretamente os vetores de controle juntamente com uma amostra densa de vetores tangentes, atuando como restrição do contorno na interpolação e assim, forçando a tangência do campo resultante. Entretanto, essa abordagem apresentou diversos problemas, cuja as soluções foram a base para o método proposto. A proposta deste trabalho consistiu, em tratar de forma independente a interpolação dos vetores de controle e a restrição do contorno produzindo, respectivamente, um campo vetorial com divergente nulo e um campo tangente. Essa abordagem pode solucionar vários problemas com relação ao método anterior e foi a base para o decorrer do trabalho.

Embora domínios côncavos não sejam um problema para o uso tradicional das RBFs, o problema abordado a influência da métrica euclidiana em todo domínio pode gerar resultados indesejados pois, os vetores de controle podem afetar regiões não desejadas. Situação análoga foi abordada por Levi e Levin (2014) e de forma semelhante, propusemos o uso de métricas intrínsecas acopladas nas RBFs a fim de considerar propriedades geométicas e topológicas do domínio. Porém, o acoplamento de novas métricas não foi possível de imediato no núcleo MVRBF, devido à dependência do cálculo das derivadas, necessitando recalcular todo o núcleo. Com isso, inicialmente, propusemos uma alternativa, através da modificação dos suportes das 
funções de base através de alguma distância interior, continuando com a métrica euclidiana no núcleo matricial porém, com melhorias a respeito das vizinhanças. Este método apresentou bons resultados mas leva ao problema de um novo parâmetro para administrar, além de não termos a influência direta de novas métricas no interpolante.

As etapas seguintes foram dedicadas ao acoplamento de novas distâncias no núcleo MVRBF: a definida por Rustamov (sem malha) e a do método FM (com malha) porém, com a falta das expressões analíticas para as derivadas dessas distâncias foram utilizadas aproximações numéricas. Infelizmente, com a distância de Rustamov, o método não apresentou bons resultados, devido a valores elevados das derivadas em algumas regiões de vários domínios testados, indicando uma característica da distância. Já com a distância do FM, o acoplamento se comportou muito bem. Os suporte das fuções de base ficaram automaticamente determinados pela nova distância resultando num controle mais intuitivo dos vetores de design.

Na etapa da soma dos campos, existe um balanceamento entre preservar o divergente nulo e forçar as linhas do campo a tangenciar a fronteira. Embora o divergente do campo resultante seja alterado, foram obtidos bons resultados qualitativos e quantitativos, viabilizando esta nova abordagem para o problema de design de campos vetoriais. Até o momento, o desempenho do método é exclusivamente prejudicado pelo cálculo das aproximações das derivadas porém, podem ser précalculadas e então o design pode ser executado em tempo real.

Como melhorias e trabalhos futuros, podemos considerar:

- Adaptar o método para domínios tridimensionais;

- Minimizar a perda do divergente nulo após a soma com o campo tangente;

- Buscar por métricas intrínsecas com formas analíticas para derivadas de segunda ordem.

Na Figura 34, temos o primeiro resultado tridimensional do método proposto utilizando uma esfera como domínio. O núcleo MVRBF é facilmente adapatado para o caso tridimensional e pela convexidade da esfera podemos utilizar apenas a distância euclidiana. 
Figura 34 - Primeiro resultado tridimensional utilizando uma esfera como domínio

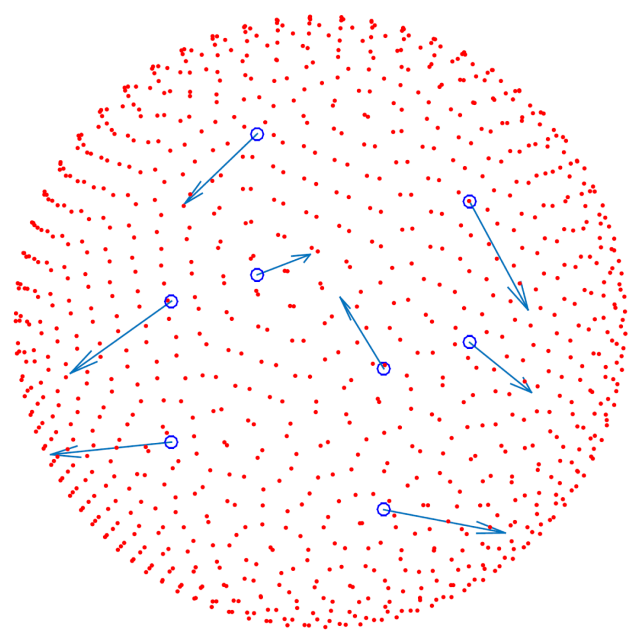

(a) Pontos da fronteira e oito vetores de controle no interior

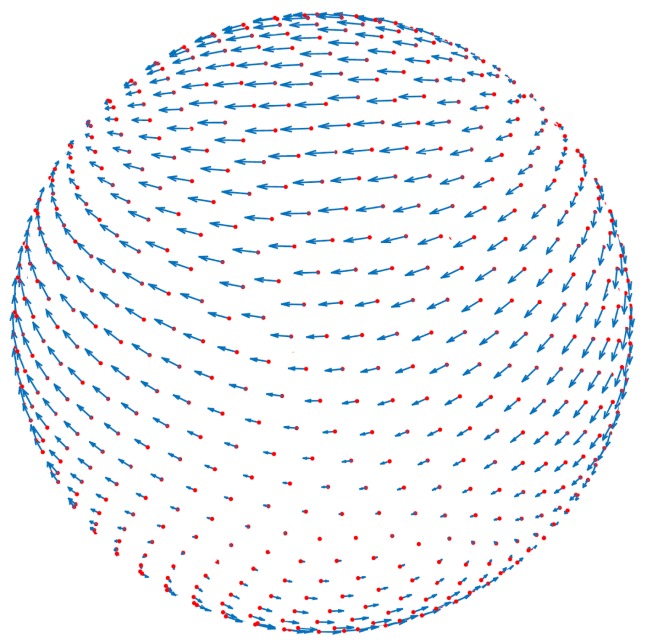

(b) Campo tangente obtido dos vetores de controle

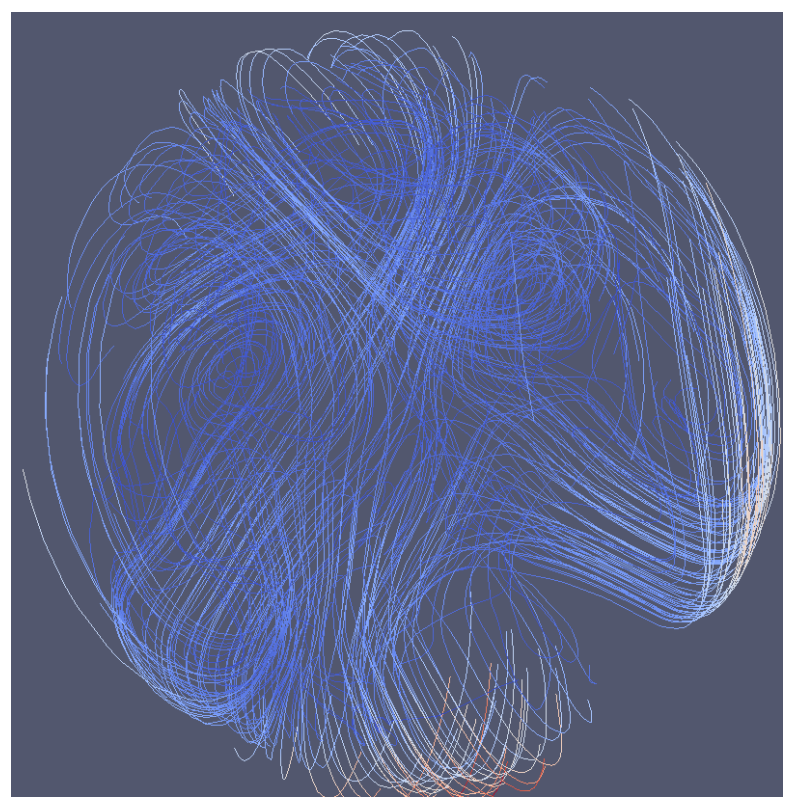

(c) Vizualização das linhas de fluxo do campo resultante

Fonte: Elaborada pelo autor. 



\section{REFERÊNCIAS}

BRIDSON, R.; HOURIHAM, J.; NORDENSTAM, M. Curl-noise for procedural fluid flow. In: ACM SIGGRAPH 2007 Papers. New York, NY, USA: ACM, 2007. (SIGGRAPH '07). Disponível em: <http://doi.acm.org/10.1145/1275808.1276435>. Citado na página 19.

BRONSTEIN, A.; BRONSTEIN, M.; KIMMEL, R. Numerical Geometry of Non-Rigid Shapes. 1. ed. [S.1.]: Springer Publishing Company, Incorporated, 2008. ISBN 0387733000, 9780387733005. Citado na página 32.

CABRAL, B.; LEEDOM, L. C. Imaging vector fields using line integral convolution. In: Proceedings of the 20th Annual Conference on Computer Graphics and Interactive Techniques. New York, NY, USA: ACM, 1993. (SIGGRAPH '93), p. 263-270. ISBN 0-89791-601-8. Disponível em: <http://doi.acm.org/10.1145/166117.166151>. Citado na página 53.

CRANE, K.; DESBRUN, M.; SCHRöDER, P. Trivial connections on discrete surfaces. Computer Graphics Forum, Blackwell Publishing Ltd, v. 29, n. 5, p. 1525-1533, 2010. ISSN 1467-8659. Disponível em: <http://dx.doi.org/10.1111/j.1467-8659.2010.01761.x>. Citado na página 20.

FASSHAUER, G. F. Meshfree Approximation Methods with MATLAB. River Edge, NJ, USA: World Scientific Publishing Co., Inc., 2007. ISBN 9789812706348, 9812706348. Citado nas páginas 25 e 26.

FISHER, M.; SCHRöDER, P.; DESBRUN, M.; HOPPE, H. Design of tangent vector fields. In: ACM SIGGRAPH 2007 Papers. New York, NY, USA: ACM, 2007. (SIGGRAPH '07). Disponível em: <http://doi.acm.org/10.1145/1275808.1276447>. Citado nas páginas 17, 18, 19 e 20 .

FUSELIER, E. Refined Error Estimates For Matrix-Valued Radial Basis Funcitons. Tese (Doutorado) - Texas A\&M University, 2006. Citado nas páginas 30 e 31.

FUSELIER, E. J.; WRIGHT, G. B. A radial basis function method for computing helmholtz-hodge decompositions. IMA Journal of Numerical Analysis, v. 37, n. 2, p. 774-797, 2017. Disponível em: <http://dx.doi.org/10.1093/imanum/drw027>. Citado na página 31.

HAN, X.; HOU, M. Quasi-interpolation for data fitting by the radial basis functions. In: Proceedings of the 5th International Conference on Advances in Geometric Modeling and Processing. Berlin, Heidelberg: Springer-Verlag, 2008. (GMP'08), p. 541-547. ISBN 3-540-79245-7, 978-3-540-79245-1. Disponível em: <http://dl.acm.org/citation.cfm?id=1792279.1792326>. Citado na página 44.

LAGE, M.; CASTRO, R.; PETRONETTO, F.; BORDIGNON, A. L.; TAVARES, G.; LEWINER, T.; LOPES, H. Support vectors learning for vector field reconstruction. In: SIBGRAPI 2009, Proceedings of the XXII Brazilian Symposium on Computer Graphics and Image Processing, Rio de Janeiro, Brazil, 11-15 October 2009. [s.n.], 2009. p. 104-111. Disponível em: $<$ https://doi.org/10.1109/SIBGRAPI.2009.20>. Citado na página 19. 
LAGE, M.; PETRONETTO, F.; PAIVA, A.; LOPES, H.; LEWINER, T.; TAVARES, G. Vector field reconstruction from sparse samples with applications. In: Sibgrapi 2006 (XIX Brazilian Symposium on Computer Graphics and Image Processing). Manaus, AM: IEEE, 2006. p. 297-304. Citado na página 19.

LEVI, Z.; LEVIN, D. Shape deformation via interior rbf. IEEE Transactions on Visualization and Computer Graphics, IEEE Educational Activities Department, Piscataway, NJ, USA, v. 20, n. 7, p. 1062-1075, jul. 2014. ISSN 1077-2626. Citado nas páginas 33, 45 e 61.

LIU, S.; WANG, C. C. L. Quasi-interpolation for surface reconstruction from scattered data with radial basis function. Comput. Aided Geom. Des., Elsevier Science Publishers B. V., Amsterdam, The Netherlands, The Netherlands, v. 29, n. 7, p. 435-447, out. 2012. ISSN 01678396. Disponível em: <http://dx.doi.org/10.1016/j.cagd.2012.03.011>. Citado na página 44.

LOWITZSCH, S. Approximation and interpolation employing divergence-free radial basis function with applications. Tese (phdthesis) - Texas A\&M University, 2002. Citado na página 43.

LOWITZSCH, S. Error estimates for matrix-valued radial basis function interpolation. J. Approx. Theory, Academic Press, Inc., Orlando, FL, USA, v. 137, n. 2, p. 238-249, dez. 2005. ISSN 0021-9045. Citado na página 31.

MACêDO, I.; CASTRO, R. Learning divergence-free and curl-free with matrix-valued kernels. Instituto Nacional de Matematica Pura e Aplicada, Brasil, Tech. Rep, 2008. Citado na página 19.

NARCOWICH, F. J.; WARD, J. D. Generalized hermite interpolation via matrix-valued conditionally positive definite functions. Math. Comput., American Mathematical Society, Boston, MA, USA, v. 63, n. 208, p. 661-687, out. 1994. ISSN 0025-5718. Citado nas páginas 28 e 61.

NARCOWICH, F. J.; WARD, J. D.; WRIGHT, G. B. Divergence-free RBFs on Surfaces. J. Fourier Anal. Appl., v. 13, p. 643-663, 2007. Citado na página 33.

PALACIOS, J.; MA, C.; CHEN, W.; WEI, L.-Y.; ZHANG, E. Tensor field design in volumes. In: SIGGRAPH ASIA 2016 Technical Briefs. New York, NY, USA: ACM, 2016. (SA '16), p. 18:118:4. ISBN 978-1-4503-4541-5. Disponível em: <http://doi.acm.org/10.1145/3005358.3005369>. Citado nas páginas 17, 18, 20 e 21.

PEYRÉ, G.; PÉCHAUD, M.; KERIVEN, R.; COHEN, L. D. Geodesic methods in computer vision and graphics. Found. Trends. Comput. Graph. Vis., Now Publishers Inc., Hanover, MA, USA, v. 5, n. 3\&\#8211;4, p. 197-397, mar. 2010. ISSN 1572-2740. Disponível em: $<$ http://dx.doi.org/10.1561/0600000029>. Citado na página 32.

RUSTAMOV, R. M.; LIPMAN, Y.; FUNKHOUSER, T. Interior distance using barycentric coordinates. In: Proceedings of the Symposium on Geometry Processing. Aire-la-Ville, Switzerland, Switzerland: Eurographics Association, 2009. (SGP '09), p. 1279-1288. Citado nas páginas 34 e 35.

SETHIAN, J. A. Fast marching methods. SIAM Rev., Society for Industrial and Applied Mathematics, Philadelphia, PA, USA, v. 41, n. 2, p. 199-235, jun. 1999. ISSN 0036-1445. Disponível em: <http://dx.doi.org/10.1137/S0036144598347059>. Citado na página 33. 
SOLOMON, J.; VAXMAN, A.; BOMMES, D. Boundary element octahedral fields in volumes. ACM Trans. Graph., ACM, New York, NY, USA, v. 36, n. 3, p. 28:1-28:16, maio 2017. ISSN 0730-0301. Disponível em: <http://doi.acm.org/10.1145/3065254>. Citado nas páginas 20 e 21.

VAXMAN, A.; CAMPEN, M.; DIAMANTI, O.; BOMMES, D.; HILDEBRANDT, K.; TECHNION, M. B.-C.; PANOZZO, D. Directional field synthesis, design, and processing. In: ACM SIGGRAPH 2017 Courses. New York, NY, USA: ACM, 2017. (SIGGRAPH '17), p. 12:1-12:30. ISBN 978-1-4503-5014-3. Disponível em: <http://doi.acm.org/10.1145/3084873. 3084921>. Citado na página 20.

WENDLAND, H. Piecewise polynomial, positive definite and compactly supported radial functions of minimal degree. Adv. in Comput. Math. 4, p. 389-396, 1995. Citado na página 26.

. Scattered Data Approximation. [S.1.]: Cambridge University Press, 2005. Citado nas páginas 24, 25, 26 e 33.

Divergence-free kernel methods for approximating the stokes problem. SIAM J. Numer. Anal., Society for Industrial and Applied Mathematics, Philadelphia, PA, USA, v. 47, n. 4, p. 3158-3179, out. 2009. ISSN 0036-1429. Citado na página 31. 


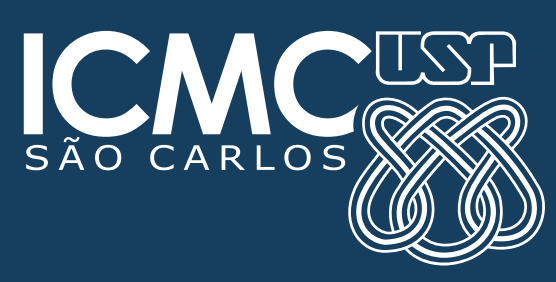

